\title{
Phenomenology of NMSSM in TeV scale mirage mediation
}

\section{Kei Hagimoto, ${ }^{a}$ Tatsuo Kobayashi, ${ }^{b}$ Hiroki Makino, ${ }^{a}$ Ken-ichi Okumura ${ }^{a}$ and Takashi Shimomura ${ }^{c}$}

${ }^{a}$ Department of Physics, Kyushu University, 744, Motooka, Nishi-ku, Fukuoka 819-0395, Japan

${ }^{b}$ Department of Physics, Hokkaido University, Kita 10, Nishi 8, Kita-ku, Sapporo 060-0810, Japan

${ }^{c}$ Faculty of Education and Culture, University of Miyazaki, 1-1, Gakuen Kibanadai Nishi, Miyazaki 889-2192, Japan

E-mail: hagimoto@higgs.phys.kyushu-u.ac.jp,

kobayashi@particle.sci.hokudai.ac.jp, makino@higgs.phys.kyushu-u.ac.jp, okumura@phys.kyushu-u.ac.jp, shimomura@cc.miyazaki-u.ac.jp

ABSTRACT: We study the next-to-minimal supersymmetric standard model (NMSSM) with the $\mathrm{TeV}$ scale mirage mediation, which is known as a solution for the little hierarchy problem in supersymmetry. Our previous study showed that $125 \mathrm{GeV}$ Higgs boson is realized with $\mathcal{O}(10) \%$ fine-tuning for $1.5 \mathrm{TeV}$ gluino ( $1 \mathrm{TeV}$ stop) mass. The $\mu$ term could be as large as $500 \mathrm{GeV}$ without sacrificing the fine-tuning thanks to a cancellation mechanism. The singlet-doublet mixing is suppressed by $\tan \beta$. In this paper, we further extend this analysis. We argue that approximate scale symmetries play a role behind the suppression of the singlet-doublet mixing. They reduce the mixing matrix to a simple form that is useful to understand the results of the numerical analysis. We perform a comprehensive analysis of the fine-tuning including the singlet sector by introducing a simple formula for the fine-tuning measure. This shows that the singlet mass of the least fine-tuning is favored by the LEP anomaly for moderate $\tan \beta$. We also discuss prospects for the precision measurements of the Higgs couplings at LHC and ILC and direct/indirect dark matter searches in the model.

KeYWORDS: Supersymmetry Phenomenology

ARXIV EPRINT: 1509.05327 


\section{Contents}

1 Introduction 1

2 TeV-scale mirage mediation 3

3 NMSSM 5

4 TeV scale mirage mediation in NMSSM $\quad 6$

4.1 Model 6

5 Spectrum and phenomenological aspects 11

$\begin{array}{lll}5.1 & \text { Higgs mass spectrum } & 12\end{array}$

$\begin{array}{lll}5.2 & \text { Fine-tuning in the electroweak symmetry breaking } & 15\end{array}$

5.3 Higgs couplings and collider signature 20

$\begin{array}{ll}5.4 & \text { Dark matter } \\ \end{array}$

6 Conclusion 33

A Soft SUSY breaking terms

\section{Introduction}

The first run of the LHC has finished successfully. The Higgs boson, the final missing piece of the standard model (SM), was discovered by ATLAS [1] and CMS [2] and its mass was measured as $m_{h} \approx 125 \mathrm{GeV}$ [3]. Subsequently, its spin, parity [4-7] and couplings to the SM bosons $[8,9]$ were measured and appeared to have the property predicted by the SM within the current experimental accuracy. Now any new physics model must predict this almost SM-like Higgs boson. On the other hand, no sign of the new particles beyond the SM was discovered. This casts a serious doubt on the naturalness of the electroweak (EW) symmetry breaking and new physics models based on it.

In particular, supersymmetry (SUSY) has been one of the most popular physics beyond the SM to solve the hierarchy problem. In the context of SUSY, the LHC Run I did not find expected superpartners, instead put the lower bound of superpartner masses as about 1.4 TeV for gluino, $1 \mathrm{TeV}$ for the first/second generation squarks [10-20] and $700 \mathrm{GeV}$ for the third generation squarks [20-37] in simplified models. Thus, the little hierarchy between the Higgs mass and superpartner masses now becomes manifest. Within the framework of the minimal supersymmetric standard model (MSSM), the $Z$-boson mass, $m_{Z}$, is obtained as

$$
m_{Z}^{2} \simeq-2 m_{H_{u}}^{2}+\frac{2}{\tan ^{2} \beta} m_{H_{d}}^{2}-2 \mu^{2}
$$


where $m_{H_{u}}^{2}$ and $m_{H_{d}}^{2}$ are the soft SUSY breaking scalar mass squared of the up-sector and down-sector Higgs fields and $\mu$ is the supersymmetric mass. For example, in the constrained MSSM the radiative corrections on $m_{H_{u}}^{2}$ are dominated by the gluino mass $M_{3}$, and obtained as $m_{H_{u}}^{2} \sim-M_{3}^{2}$. Thus, if the gluino and stop masses are of $\mathcal{O}(1) \mathrm{TeV}$, we need fine-tuning among $m_{H_{u}}^{2}, m_{H_{d}}^{2}$ and $\mu^{2}$ to realize the experimental value of $m_{Z}$. Furthermore, the stop mass is required to be of $\mathcal{O}(1) \mathrm{TeV}$ or larger to lead to the Higgs mass $m_{h} \approx 125 \mathrm{GeV}$. If we further rely on the naturalness as a guiding principle, we need a mechanism of SUSY breaking which reconciles this little hierarchy with the notion of naturalness.

The mirage mediation is one of the interesting mediation mechanisms of SUSY breaking [38-41] (also see [42-63]). It is a mixture of the modulus mediation [64-67] and the anomaly mediation $[68,69]$ with a certain ratio. In the mirage mediation, the radiative corrections and the anomaly mediation contributions cancel each other at a certain energy scale, where SUSY spectrum appears as that of the pure modulus mediation. Such an energy scale is called the mirage scale. TeV scale mirage mediation sets this scale at $\mathrm{TeV}$ scale and it was pointed out that the $\mathrm{TeV}$ scale mirage mediation can significantly ameliorate the above fine-tuning problem of the MSSM [70-72]. ${ }^{1}$

Although the $\mathrm{TeV}$ scale mirage mediation is an attractive scenario to solve the little hierarchy problem, there are two unsatisfactory features in the MSSM. First, a typical mass scale of the $B$-term is the gravitino mass $(10-100 \mathrm{TeV})$ and a delicate cancellation between different contributions is required to realize the correct EW symmetry breaking $[40,70,86]$. Second, $125 \mathrm{GeV}$ Higgs mass is still difficult to achieve by $\mathcal{O}(10) \%$ tuning of parameters because the $A$-term for the top Yukawa coupling is fixed by the model, which prevents the stop mixing from taking the optimal value known as the maximal mixing. A simple solution for these problems is an extension to the next-to-minimal supersymmetric standard model (NMSSM) [40].

The NMSSM is the minimal extension of the MSSM by adding a singlet superfield $S$ [87-95] (see for review e.g. [96, 97]). Here we impose the $Z_{3}$ symmetry, which does not allow the $\mu$-term, $\mu H_{u} H_{d}$, in the superpotential, where $H_{u}$ and $H_{d}$ denote the up and down-sector Higgs superfields. Instead, the term such as $\lambda S H_{u} H_{d}$ is allowed. After the scalar component of $S$ develops its vacuum expectation value (VEV), the effective $\mu$-term is generated as $\mu=\lambda\langle S\rangle$. In the $Z_{3}$ symmetric NMSSM, all of the dimensionful parameters are originated from SUSY breaking. Hence, the value of $\mu$ is also obtained by SUSY breaking effects. That gives us a solution for the so-called $\mu$-problem [98]. The $B$-term in the MSSM is replaced with the $A$-term, $A_{\lambda}$ which has a scale of gaugino mass in the mirage mediation and solves the first problem in the MSSM. The NMSSM has an additional Higgs quartic coupling, $\lambda^{2}$, in the scalar potential, which is helpful to increase the tree-level Higgs mass compared with one in the MSSM. This ameliorates the second problem if $\tan \beta$ is small. Also the mixing with the singlet and doublet helps to raise the Higgs mass if the singlet is light, although it tightens the constraint on the singlet from the LEP Higgs search [99] (for recent studies on the mixing effect in NMSSM, see [100-113]).

\footnotetext{
${ }^{1}$ Certain relations among the soft SUSY breaking parameters at the unification scale may be useful to ameliorate fine-tuning [73-85].
} 
The TeV scale mirage mediation was applied to the NMSSM in ref. [103]. It was found that the fine-tuning is improved in the parameter region to realize $m_{h} \approx 125 \mathrm{GeV}$. The effective $\mu$ can be considerably larger than the EW scale without sacrificing the fine-tuning due to a cancellation mechanism, although it is assumed to be around the EW scale in conventional natural SUSY models [114-122]. ${ }^{2}$ The mixing between the singlet and doublet Higgs bosons is suppressed. In addition, the Higgs sector as well as the neutralino sector has a rich structure compared with one in the MSSM by adding the singlet. That leads to interesting aspects as shown in ref. [103]. ${ }^{3}$ In this paper, we further extend the analysis in [103] including new phenomenological studies. We show that approximate scale symmetries play an important roll to suppress the singlet-doublet mixing in the NMSSM. We discuss the suppression holds as far as $\kappa \approx 0$ and $m_{S, H_{u}}^{2} \ll m_{H_{d}}^{2}$. We introduce a simple formula for the fine-tuning measures and study the fine-tuning of the EW symmetry breaking in detail. We show that the singlet mass of the least fine-tuning is favored by the LEP anomaly [99, 103, $125-132]$ when $\tan \beta$ is moderately large. We also study other phenomenological aspects such as the precision measurement of the Higgs couplings and the dark matter search.

This paper is organized as follows. In section 2, we give a brief review on the mirage mediation, and the $\mathrm{TeV}$ scale mirage mediation. We introduce the $Z_{3}$ symmetric NMSSM in section 3 and apply the $\mathrm{TeV}$ scale mirage mediation in section 4 . In section 5 , we study phenomenological aspects such as the mass spectrum, fine-tuning in the EW symmetry breaking, Higgs couplings and dark matter. Section 6 is devoted to conclusion and discussion. In appendix A, we show explicitly initial conditions of soft parameters, which are induced through the mirage mediation in the NMSSM.

\section{TeV-scale mirage mediation}

Here we give a brief review on the mirage mediation [38, 39]. The mirage mediation is the mixture of the modulus mediation and the anomaly mediation with a certain ratio, which would be determined by the modulus stabilization mechanism and SUSY breaking mechanism. In the mirage mediation, the gaugino masses are written by

$$
M_{a}\left(M_{\mathrm{GUT}}\right)=M_{0}+\frac{m_{3 / 2}}{8 \pi^{2}} b_{a} g_{a}^{2}
$$

where $g_{a}$ and $b_{a}$ are the gauge couplings and their $\beta$ function coefficients, and $m_{3 / 2}$ denotes the gravitino mass. We assume that the initial conditions of our SUSY breaking parameters are input at the GUT scale, $M_{\mathrm{GUT}}=2 \times 10^{16} \mathrm{GeV}$. The first term, $M_{0}$, in the right hand side denotes the gaugino mass due to the pure modulus mediation, while the second term corresponds to the anomaly mediation contribution. In addition, we can write the soft scalar mass $m_{i}$ of matter fields $\phi^{i}$ and the so-called $A$-terms of $\phi^{i} \phi^{j} \phi^{k}$ corresponding to the Yukawa couplings $y_{i j k}$ as

$$
A_{i j k}\left(M_{\mathrm{GUT}}\right)=a_{i j k} M_{0}-\left(\gamma_{i}+\gamma_{j}+\gamma_{k}\right) \frac{m_{3 / 2}}{8 \pi^{2}},
$$

\footnotetext{
${ }^{2}$ As a different approach in this direction, see [123].

${ }^{3}$ See also ref. [124].
} 


$$
m_{i}^{2}\left(M_{\mathrm{GUT}}\right)=c_{i} M_{0}^{2}-\dot{\gamma}_{i}\left(\frac{m_{3 / 2}}{8 \pi^{2}}\right)^{2}-\frac{m_{3 / 2}}{8 \pi^{2}} M_{0} \theta_{i}
$$

where

$$
\begin{aligned}
\gamma_{i} & =2 \sum_{a} g_{a}^{2} C_{2}^{a}\left(\phi^{i}\right)-\frac{1}{2} \sum_{j k}\left|y_{i j k}\right|^{2}, \\
\theta_{i} & =4 \sum_{a} g_{a}^{2} C_{2}^{a}\left(\phi^{i}\right)-\sum_{j k} a_{i j k}\left|y_{i j k}\right|^{2}, \\
\dot{\gamma}_{i} & =8 \pi^{2} \frac{d \gamma_{i}}{d \ln \mu_{R}} .
\end{aligned}
$$

Here, $\gamma_{i}$ denotes the anomalous dimensions of $\phi^{i}$ and $C_{2}^{a}\left(\phi^{i}\right)$ denotes the quadratic Casimir corresponding to the representation of the matter field $\phi^{i}$. In the right hand side, $a_{i j k} M_{0}$ and $c_{i} M_{0}^{2}$ denote the A-term and soft scalar masses squared due to the pure modulus mediation. These coefficients, $a_{i j k}$ and $c_{i}$, are determined by modulus-dependence of the Kähler metric of $\phi^{i}, \phi^{j}$ and $\phi^{k}$ as well as Yukawa couplings. Indeed, by using the treelevel Kähler metric, the coefficient $c_{i}$ is explicitly calculated as a fractional number such as $0,1,1 / 2,1 / 3$, etc. $[64-67,72,134]$. We would have $\mathcal{O}\left(1 / 8 \pi^{2}\right)$ of corrections on $c_{i}$ due to the one-loop corrections on the Kähler metric [135]. Such a correction would be important when $c_{i}=0$, but that is model-dependent. Here, we consider the case with

$$
a_{i j k}=c_{i}+c_{j}+c_{k} .
$$

Such a relation is often satisfied for $\mathcal{O}(1)$ of Yukawa couplings in explicit string-derived supergravity models [64-67]. ${ }^{4}$

It is convenient to use the following parameter [40],

$$
\alpha \equiv \frac{m_{3 / 2}}{M_{0} \ln \left(M_{p l} / m_{3 / 2}\right)},
$$

to represent the ratio of the anomaly mediation to the modulus mediation. Here $M_{p l}$ is the reduced Planck scale.

The mirage mediation has a very important energy scale, that is, the mirage scale defined by,

$$
M_{\mathrm{mir}}=\frac{M_{\mathrm{GUT}}}{\left(M_{p l} / m_{3 / 2}\right)^{\alpha / 2}} .
$$

The above spectrum of the gaugino masses at $M_{\mathrm{GUT}}$ leads to [40],

$$
M_{a}\left(M_{\mathrm{mir}}\right)=M_{0},
$$

at the mirage scale. That is, the anomaly mediation contributions and the radiative corrections cancel each other, and the pure modulus mediation appears at the mirage scale. Furthermore, the $A$-terms and the scalar mass squared also satisfy

$$
A_{i j k}\left(M_{\text {mir }}\right)=\left(c_{i}+c_{j}+c_{k}\right) M_{0}, \quad m_{i}^{2}\left(M_{\text {mir }}\right)=c_{i} M_{0}^{2},
$$

\footnotetext{
${ }^{4}$ See also [136, 137].
} 
if the corresponding Yukawa couplings are small enough or if the following conditions are satisfied,

$$
a_{i j k}=c_{i}+c_{j}+c_{k}=1
$$

for non-vanishing Yukawa couplings, $y_{i j k}[40]$.

When $\alpha=2$, the mirage scale $M_{\text {mir }}$ is around $1 \mathrm{TeV}$. Then, the above spectrum (2.7) and (2.8) is obtained around the $\mathrm{TeV}$ scale. That is the $\mathrm{TeV}$ scale mirage mediation scenario. In particular, there would appear a large gap between $M_{0}$ and the scalar mass $m_{i}$ with $c_{i} \approx 0$. We will apply the TeV scale mirage scenario to the NMSSM in section 4 .

In the $\mathrm{TeV}$ scale mirage scenario, the stop mass squared becomes negative at high energy [138], while it is positive at low energy below $10^{6} \mathrm{GeV}$. Thus, the vacuum which breaks the EW symmetry at the EW scale might be a local minimum, but instead there would be a color and/or charge breaking vacuum with field values larger than $10^{6} \mathrm{GeV}$. Here, we assume the thermal history of the Universe such that field values remain around the origin until the EW scale temperature. In addition, we need to confirm that the tunneling rate is small enough, i.e. less than the Hubble expansion rate. In refs. [139-141], it has been shown that such a rate is small enough, as long as the squark/slepton masses squared are vanishing or positive around $10^{4} \mathrm{GeV}$. This condition is satisfied in our $\mathrm{TeV}$ scale mirage mediation scenario.

\section{$3 \quad$ NMSSM}

Here, we briefly review on the NMSSM, in particular its Higgs sector. We extend the MSSM by adding a singlet chiral multiplet $S$ and imposing a $Z_{3}$ symmetry. Then, the superpotential of the Higgs sector is written as

$$
W_{\mathrm{Higgs}}=-\lambda S H_{u} H_{d}+\frac{\kappa}{3} S^{3} .
$$

Here and hereafter, for $S, H_{u}$ and $H_{d}$ we use the convention that the superfield and its lowest component are denoted by the same letter. The full superpotential also includes the Yukawa coupling terms between the matter fields and the Higgs fields, which are the same as those in the MSSM.

The following soft SUSY breaking terms in the Higgs sector are induced,

$$
V_{\text {soft }}=m_{H_{u}}^{2}\left|H_{u}\right|^{2}+m_{H_{d}}^{2}\left|H_{d}\right|^{2}+m_{S}^{2}|S|^{2}-\lambda A_{\lambda} S H_{u} H_{d}+\frac{\kappa}{3} A_{\kappa} S^{3}+\text { h.c. }
$$

Then, the scalar potential of the neutral Higgs fields is given as

$$
\begin{aligned}
V= & \lambda^{2}|S|^{2}\left(\left|H_{d}^{0}\right|^{2}+\left|H_{u}^{0}\right|^{2}\right)+\left|\kappa S^{2}-\lambda H_{d}^{0} H_{u}^{0}\right|^{2}+V_{D} \\
& +m_{H_{u}}^{2}\left|H_{u}^{0}\right|^{2}+m_{H_{d}}^{2}\left|H_{d}^{0}\right|^{2}+m_{S}^{2}|S|^{2}-\lambda A_{\lambda} S H_{u}^{0} H_{d}^{0}+\frac{\kappa}{3} A_{\kappa} S^{3}+h . c .,
\end{aligned}
$$

with

$$
V_{D}=\frac{1}{8}\left(g_{1}^{2}+g_{2}^{2}\right)\left(\left|H_{d}^{0}\right|^{2}-\left|H_{u}^{0}\right|^{2}\right)^{2},
$$

where $g_{1}$ and $g_{2}$ denote the gauge couplings of $\mathrm{U}(1)_{\mathrm{Y}}$ and $\mathrm{SU}(2)$. Similarly, there appear the soft SUSY breaking terms including squarks and sleptons as well as gaugino masses. These are the same as those in the MSSM. 
The minimum of the Higgs potential is obtained by analyzing the stationary conditions of the Higgs potential,

$$
\begin{aligned}
\frac{\partial V}{\partial H_{d}^{0}}= & \lambda^{2} v \cos \beta\left(s^{2}+v^{2} \sin ^{2} \beta\right)-\lambda \kappa v s^{2} \sin \beta+\frac{1}{4} g^{2} v^{3} \cos \beta \cos 2 \beta \\
& +m_{H_{d}}^{2} v \cos \beta-\lambda A_{\lambda} v s \sin \beta=0, \\
\frac{\partial V}{\partial H_{u}^{0}}= & \lambda^{2} v \sin \beta\left(s^{2}+v^{2} \cos ^{2} \beta\right)-\lambda \kappa v s^{2} \cos \beta-\frac{1}{4} g^{2} v^{3} \sin \beta \cos 2 \beta \\
& +m_{H_{u}}^{2} v \sin \beta-\lambda A_{\lambda} v s \cos \beta=0, \\
\frac{\partial V}{\partial S}= & \lambda^{2} s v^{2}+2 \kappa^{2} s^{3}-\lambda \kappa v^{2} s \sin 2 \beta+m_{S}^{2} s-\frac{1}{2} \lambda A_{\lambda} v^{2} \sin 2 \beta+\kappa A_{\kappa} s^{2}=0,
\end{aligned}
$$

where $g^{2}=g_{1}^{2}+g_{2}^{2}$. Here, we denote VEVs as

$$
v^{2}=\left|\left\langle H_{d}^{0}\right\rangle\right|^{2}+\left|\left\langle H_{u}^{0}\right\rangle\right|^{2}, \quad \tan \beta=\frac{\left\langle H_{u}^{0}\right\rangle}{\left\langle H_{d}^{0}\right\rangle}, \quad s=\langle S\rangle .
$$

Using the above stationary conditions, we obtain the $Z$ boson mass $m_{Z}^{2}=\frac{1}{2} g^{2} v^{2}$ as

$$
m_{Z}^{2}=\frac{1-\cos 2 \beta}{\cos 2 \beta} m_{H_{u}}^{2}-\frac{1+\cos 2 \beta}{\cos 2 \beta} m_{H_{d}}^{2}-2 \mu^{2}
$$

where $\mu=\lambda s$. For $\tan \beta \gg 1$, this equation becomes eq. (1.1). That is, this relation is the same as the one in the MSSM. Thus, the natural values of $\left|m_{H_{u}}\right|$ and $|\mu|$ would be of $\mathcal{O}(100) \mathrm{GeV}$. Furthermore, the natural value of $\left|m_{H_{d}}\right| / \tan \beta$ would be of $\mathcal{O}(100) \mathrm{GeV}$ or smaller. Alternatively, $|\mu|$ and $\left|m_{H_{d}}\right| / \tan \beta$ could be larger than $\mathcal{O}(100) \mathrm{GeV}$ when $\mu^{2}$ and $m_{H_{d}}^{2} / \tan ^{2} \beta$ are canceled each other in the above relation at a certain level. Even in such a case, $\left|m_{H_{u}}\right|$ would be naturally of $\mathcal{O}(100) \mathrm{GeV}$. On the other hand, other sfermion masses as well as gaugino masses must be heavy as the recent LHC results suggested. To realize such a spectrum, we apply the $\mathrm{TeV}$ scale mirage mediation in the next section, where we take $c_{H_{u}}=0$ to realize a suppressed value of $\left|m_{H_{u}}\right|$ compared with $M_{0}$.

\section{TeV scale mirage mediation in NMSSM}

Here, we apply the TeV scale mirage mediation scenario to the NMSSM and study its phenomenological aspects.

\subsection{Model}

Soft SUSY breaking terms are obtained through the generic formulas (2.1) and (2.2) with taking $\alpha=2$. For concreteness, we give explicit results of all the soft SUSY breaking terms for the NMSSM in appendix A. We concentrate on the Higgs sector as well as gauginos and stops.

A concrete model in the mirage mediation is fixed by choosing $c_{i}$. We consider the following values of $c_{i}[103]$,

$$
c_{H_{d}}=1, \quad c_{H_{u}}=0, \quad c_{S}=0, \quad c_{t_{L}}=c_{t_{R}}=\frac{1}{2}
$$


up to one-loop corrections for $H_{d}, H_{u}, S$, and left and right-handed (s)top fields, respectively. This is the same assignment as the pattern II in ref. [72] for the MSSM except for $c_{S}$. Then, the soft parameters due to only modulus mediation contribution are given by

$$
\begin{aligned}
\left(A_{t}\right)_{\text {modulus }} & =\left(A_{\lambda}\right)_{\text {modulus }}=M_{0}, & \left(A_{\kappa}\right)_{\text {modulus }}=0, \\
\left(m_{H_{d}}^{2}\right)_{\text {modulus }} & =M_{0}^{2}, & \left(m_{\tilde{t}_{L}}^{2}\right)_{\text {modulus }}=\left(m_{\tilde{t}_{R}}^{2}\right)_{\text {modulus }}=\frac{1}{2} M_{0}^{2}, \\
\left(m_{H_{u}}^{2}\right)_{\text {modulus }} & =\left(m_{S}^{2}\right)_{\text {modulus }}=0, &
\end{aligned}
$$

up to one-loop corrections. The above assignment of $c_{i}$ (4.1) satisfies the condition, (2.9) for the top Yukawa coupling and the coupling $\lambda$, but not for the coupling $\kappa$. However, we do not consider a large value of $\kappa$ to avoid the blow-up of $\kappa$ and $\lambda$ as will be shown later. Thus, we obtain the following values,

$$
\begin{aligned}
A_{t} & \approx A_{\lambda} \approx M_{0}, \\
m_{H_{d}}^{2} & \approx M_{0}^{2}, \quad m_{\tilde{t}_{L}}^{2} \approx m_{\tilde{t}_{R}}^{2} \approx \frac{1}{2} M_{0}^{2},
\end{aligned}
$$

up to $\mathcal{O}\left(\kappa^{2} / 8 \pi^{2}\right)$ at the $\mathrm{TeV}$ scale.

Similarly, at the $\mathrm{TeV}$ scale we can obtain

$$
m_{H_{u}}^{2} \approx 0, \quad m_{S}^{2} \approx 0
$$

up to $\mathcal{O}\left(M_{0}^{2} / 8 \pi^{2}\right)$, and

$$
A_{\kappa} \approx 0,
$$

up to $\mathcal{O}\left(M_{0} / 8 \pi^{2}\right)$. That is, values of $\left|A_{\kappa}\right|^{2}, m_{H_{u}}^{2}$ and $m_{S}^{2}$ are suppressed compared with $M_{0}^{2}$, and their explicit values depend on the one-loop corrections on the Kähler metric. Thus, we use $A_{\kappa}$ as a free parameter, which must be small compared with $M_{0}$. In addition, we determine the values of $m_{H_{u}}^{2}, m_{S}^{2}$ and $\mu(=\lambda s)$ at the EW scale from the stationary conditions, (3.5), where we use the experimental value $m_{Z}=\frac{1}{\sqrt{2}} g v=91.19 \mathrm{GeV}$ and $\tan \beta$ as a free parameter.

Through the above procedure, the parameters, $m_{H_{u}}^{2}, m_{S}^{2}$ and $\mu$, at the EW scale are expressed by $\tan \beta, m_{H_{d}}^{2}, A_{\lambda}$ as follows, ${ }^{5}$

$$
\begin{aligned}
\mu & =\lambda\langle S\rangle=\frac{A_{\lambda} \tan \beta}{2\left(1-\frac{\kappa}{\lambda} \tan \beta\right)}\{1-\sqrt{1-4 X}\}, \\
m_{S}^{2} & =-2\left(\frac{\kappa}{\lambda}\right)^{2} \mu^{2}-\left(\frac{\kappa}{\lambda}\right) A_{\kappa} \mu+\frac{\lambda^{2}}{g^{2}} m_{Z}^{2}\left\{\left(\frac{A_{\lambda}}{\mu}+2 \frac{\kappa}{\lambda}\right) \sin 2 \beta-2\right\}, \\
m_{H_{u}}^{2} & =\frac{\tan ^{2} \beta-1}{\tan ^{2} \beta}\left(\frac{m_{H_{d}}^{2}}{\tan ^{2} \beta-1}-\mu^{2}-\frac{m_{Z}^{2}}{2}\right),
\end{aligned}
$$

where,

$$
X=\frac{m_{H_{d}}^{2}\left(1-\frac{\kappa}{\lambda} \tan \beta\right)}{A_{\lambda}^{2} \tan ^{2} \beta}\left\{1+\frac{\tan ^{2} \beta}{\tan ^{2} \beta+1}\left(\frac{2 \lambda^{2}}{g^{2}}-\frac{\tan ^{2} \beta-1}{2 \tan ^{2} \beta}\right) \frac{m_{Z}^{2}}{m_{H_{d}}^{2}}\right\} .
$$

\footnotetext{
${ }^{5} \mathrm{We}$ are only interested in the natural spectrum. Thus we discard another solution of the quadratic equation, $\mu \approx A_{\lambda} \tan \beta / 2$.
} 
For $\tan \beta \gg \max (1, \kappa / \lambda)$, these parameters are approximated as

$$
\begin{aligned}
\mu & =\lambda\langle S\rangle \sim \frac{m_{H_{d}}^{2}}{A_{\lambda} \tan \beta}, \\
m_{S}^{2} & \sim-2\left(\frac{\kappa}{\lambda}\right)^{2}\left(\frac{m_{H_{d}}^{2}}{A_{\lambda} \tan \beta}\right)^{2}-\left(\frac{\kappa}{\lambda}\right) A_{\kappa}\left(\frac{m_{H_{d}}^{2}}{A_{\lambda} \tan \beta}\right)+2 \frac{\lambda^{2}}{g^{2}} \frac{A_{\lambda}^{2}}{m_{H_{d}}^{2}} m_{Z}^{2}, \\
m_{H_{u}}^{2} & \sim \frac{m_{H_{d}}^{2}}{\tan ^{2} \beta}-\frac{m_{H_{d}}^{4}}{A_{\lambda}^{2} \tan ^{2} \beta}-\frac{m_{Z}^{2}}{2} .
\end{aligned}
$$

These formulas tell that the leading terms in the expansion by $\tan \beta$ are $\mathcal{O}\left(m_{Z}^{2}\right)$ for $m_{S}^{2}$ and $m_{H_{u}}^{2}$ and the next-to-leading terms are $\mathcal{O}\left(M_{0}^{2} / \tan ^{2} \beta\right)$ because $m_{H_{d}} \simeq A_{\lambda} \simeq M_{0}$. If $\tan \beta=\mathcal{O}(10)$, the values of $\mu$ and $\left|m_{H_{u}}\right|$ could be of $\mathcal{O}(100) \mathrm{GeV}$ while the other masses of the superpartners are of $\mathcal{O}\left(M_{0}\right)=\mathcal{O}(1) \mathrm{TeV}$. Thus, the fine-tuning problem can be ameliorated. Actually, the first and the second terms in the last equation cancel each other for our choice of $c_{i}$. The next leading contributions are of $\mathcal{O}\left(m_{H_{d}}^{2} / \tan ^{4} \beta\right)$ or $\mathcal{O}\left(m_{H_{d}}^{2} \mu / \tan ^{2} \beta A_{\lambda}\right)$. Then, $m_{Z}^{2}$ is almost determined by $m_{H_{u}}^{2}$ alone and insensitive to $\mu \approx M_{0} / \tan \beta$. This means that $\tan \beta \approx 3$ is enough to obtain the fine-tuning of $\left|\partial \ln m_{Z}^{2} / \partial \ln m_{H_{u}}^{2}\right|^{-1}=m_{Z}^{2} / 2 m_{H_{u}}^{2}=\mathcal{O}(100) \%$ for $M_{0} \approx 1 \mathrm{TeV}$. Therefore $\mu$ can be as heavy as $\mathcal{O}(400) \mathrm{GeV}$ without sacrificing the fine-tuning. This cancellation originates in the structure of the doublet mass matrix,

$$
\mathcal{L}_{M}=-\left(H_{d}, H_{u}^{*}\right) \mathcal{M}_{H}^{2}\left(\begin{array}{c}
H_{d}^{*} \\
H_{u}
\end{array}\right)
$$

where

$$
\mathcal{M}_{H}^{2}=\left(\begin{array}{cc}
m_{H_{d}}^{2}+\mu^{2} & -A_{\lambda} \mu \\
-A_{\lambda} \mu & m_{H_{u}}^{2}+\mu^{2}
\end{array}\right) \approx\left(\begin{array}{cc}
M_{0}^{2}+\mu^{2} & -M_{0} \mu \\
-M_{0} \mu & \mu^{2}
\end{array}\right) .
$$

Calculating the determinant of the mass matrix, it can be easily checked that the modulus mediated contribution $M_{0}$ cancels, $\operatorname{det}\left(\mathcal{M}_{H}^{2}\right) \approx \mu^{4}$ while the trace of the mass matrix is $M_{0}^{2}+2 \mu^{2}$. This means that the heavy mode has mass of $\mathcal{O}\left(M_{0}\right)$ and the mass of the light mode is suppressed as $\mu^{2} / M_{0} \approx \mu / \tan \beta$. Therefore a flat direction appears along $H_{u} / H_{d} \approx M_{0} / \mu \approx \tan \beta$. This mechanism was previously observed in [72] in the context of the MSSM where $A_{\lambda}$ is replaced with the $B$-term. In the mirage mediation, $B$-term is a remnant of the fine-tuned cancellation between the terms of $\mathcal{O}\left(m_{3 / 2}\right)$. Thus the relation $m_{H_{d}} \approx B$ is subject to uncontrolled corrections. However, in the NMSSM, the relation is well controlled up to the leading contribution of the modulus mediation.

This cancellation is also important for raising the SM-like Higgs boson mass radiatively. In the NMSSM, singlino-higgsino loop with $\lambda=\mathcal{O}(1)$ could contribute to the radiative correction of the Higgs boson mass in addition to the top quark loop [104, 142]. If the singlet scalar is as light as the singlino or higgsino, this correction is canceled by the scalar loops because of SUSY. However, the structure of the mass matrix (4.10) tells the coupling $|S|^{2} h^{2}\left(\sim \mu^{2} h^{2}\right)$ vanishes due to the SUSY breaking effect. This means the correction 
could be sizable even if the singlet scalar is light as in our case. The renormalization group equation in the effective theory roughly gives

$$
\begin{aligned}
\Delta m_{h}^{2} \simeq \frac{v^{2}}{16 \pi^{2}} & {\left[-4 \lambda^{2} \frac{m_{h}^{2}}{v^{2}}+8 \lambda^{4}-4 \lambda^{4}\left(1-\frac{A_{\lambda}^{2}}{m_{H}^{2}}\right)\right.} \\
& \left.\quad-16 \lambda^{4} \frac{A_{\lambda}^{2}}{m_{H}^{2}}\left(1-\frac{A_{\lambda}^{2}}{m_{H}^{2}}\right) \frac{1}{\tan ^{2} \beta}\right] \ln \left(\frac{m_{H}}{m_{h}}\right) \\
\simeq & \frac{v^{2}}{16 \pi^{2}}\left[-4 \lambda^{2} \frac{m_{h}^{2}}{v^{2}}+8 \lambda^{4}\right] \ln \left(\frac{m_{H}}{m_{h}}\right), \\
\simeq & (56 \mathrm{GeV})^{2} \lambda^{2}\left(\lambda^{2}-0.26\right)\left(1+1.1 \log _{10}\left[\frac{m_{H}}{1 \mathrm{TeV}}\right]\right),
\end{aligned}
$$

where $m_{h}\left(m_{H}\right)$ is the mass of the light (heavy) doublet. In our case, $m_{H} \approx A_{\lambda} \approx M_{0}$. This correction amounts $\sim 5 \mathrm{GeV}$ for $m_{h}=m_{Z}$ and $\lambda=0.7$.

Potentially, the mixing between the light doublet and the singlet may invalidate the above discussion on the hierarchy of the Higgs boson masses. In models with $m_{S}^{2} \approx 0$ and $\kappa \ll 1$, this mixing is automatically suppressed by $\lambda v / A_{\lambda} / \tan \beta$ if we neglect the gauge interaction. (This suppression is noticed in [100] without a reason.) In this limit, the Higgs action has the following approximate scaling symmetry:

$$
\begin{aligned}
H_{u}(x) & =e^{2 \phi} H_{u}^{\prime}\left(e^{\phi} x\right) \\
H_{d}(x) & =e^{2 \phi} H_{d}^{\prime}\left(e^{\phi} x\right) \\
S(x) & =S^{\prime}\left(e^{\phi} x\right),
\end{aligned}
$$

where $\phi$ is a free scaling parameter. This symmetry is explicitly broken by the Kähler potential,

$$
\mathcal{K}_{S}=S^{\dagger} S,
$$

the D-term potential and all the kinetic terms. After the Higgs bosons develop the vacuum expectation values, the symmetry is spontaneously broken and the Nambu-Goldstone boson appears, which corresponds to the light doublet. ${ }^{6}$ Thus it is inherently mass eigenstate without the singlet component. The symmetry breaking Kähler potential generates the F-term potential:

$$
V_{F}^{S}=\lambda^{2}\left|H_{u}\right|^{2}\left|H_{d}\right|^{2},
$$

which does not affect the singlet sector at all. This potential gives additional terms in the doublet mass matrix which we neglected before:

$$
\frac{\partial^{2} V_{F}^{S}}{\partial H_{u}^{*} \partial H_{u}}=\lambda^{2}\left|H_{u}\right|^{2}, \quad \frac{\partial^{2} V_{F}^{S}}{\partial H_{d}^{*} \partial H_{d}}=\lambda^{2}\left|H_{d}\right|^{2}, \quad \frac{\partial^{2} V_{F}^{S}}{\partial H_{d}^{*} \partial H_{u}}=\lambda^{2} H_{u}^{*} H_{d} .
$$

The contributions to the diagonal elements are eliminated by the minimization conditions (3.5a), (3.5b). The CP even part of the mass matrix is given by

$$
\mathcal{M}_{S}^{2} \equiv \frac{\partial^{2} V}{\partial \phi_{i} \partial \phi_{j}}
$$

\footnotetext{
${ }^{6}$ Similar symmetry can be defined in MSSM, which forbids all the quartic potential. In such a case, the non-trivial VEV is not possible unless the mass parameters are tuned so that a massless mode appears in that direction.
} 


$$
\begin{aligned}
= & \left(\begin{array}{ccc}
A_{\lambda}^{2} \sin ^{2} \beta & \left(-A_{\lambda}^{2}+2 \lambda^{2} v^{2}\right) \sin \beta \cos \beta & \lambda A_{\lambda} v \cos 2 \beta \sin \beta \\
\left(-A_{\lambda}^{2}+2 \lambda^{2} v^{2}\right) \sin \beta \cos \beta & A_{\lambda}^{2} \cos ^{2} \beta & -\lambda A_{\lambda} v \cos 2 \beta \cos \beta \\
\lambda A_{\lambda} v \cos 2 \beta \sin \beta & -\lambda A_{\lambda} v \cos 2 \beta \cos \beta & \lambda^{2} v^{2}
\end{array}\right) \\
= & \left(\begin{array}{ccc}
\cos \beta & -\sin \beta & 0 \\
\sin \beta & \cos \beta & 0 \\
0 & 0 & 1
\end{array}\right)\left(\begin{array}{ccc}
0 & 0 & 0 \\
0 & A_{\lambda}^{2} & -\lambda A_{\lambda} v \cos 2 \beta \\
0-\lambda A_{\lambda} v \cos 2 \beta & \lambda^{2} v^{2}
\end{array}\right)\left(\begin{array}{ccc}
\cos \beta & \sin \beta & 0 \\
-\sin \beta \cos \beta & 0 \\
0 & 0 & 1
\end{array}\right) \\
& +\left(\begin{array}{ccc}
0 & \lambda^{2} v^{2} \sin 2 \beta & 0 \\
\lambda^{2} v^{2} \sin 2 \beta & 0 & 0 \\
0 & 0 & 0
\end{array}\right),
\end{aligned}
$$

where $\phi_{i}=\sqrt{2}\left(R e H_{d}-\left\langle H_{d}\right\rangle, R e H_{u}-\left\langle H_{u}\right\rangle, \operatorname{Re} S-\langle S\rangle\right)$ and we used the minimization condition (3.5c), e.g. $\mu=\frac{1}{2} A_{\lambda} \sin 2 \beta$ which is not affected by the gauge coupling. The last term which corresponds to the off-diagonal contribution in (4.15) mixes the light and heavy doublets and slightly changes the diagonalization angle of the $\mathrm{CP}$ even mass matrix, $\beta^{\prime} \equiv \pi / 2-\alpha$ from the direction of the Nambu-Goldstone boson, $\beta$ as

$$
\frac{1}{2} \tan 2 \beta^{\prime}=\frac{1}{2} \tan 2 \beta\left(1-2 \frac{\lambda^{2} v^{2}}{A_{\lambda}^{2}}\right), \quad \frac{2 \delta \beta}{\sin 2 \beta \cos 2 \beta} \approx-2 \frac{\lambda^{2} v^{2}}{A_{\lambda}^{2}},
$$

where $\delta \beta \equiv \beta^{\prime}-\beta$. This small rotation generates a non-zero entry in the singlet-light doublet mixing terms (1-3, 3-1 elements) in the CP even Higgs mass matrix. The 2-3 (3-2) element is $-\lambda A_{\lambda} v \cos 2 \beta$. Thus the rotation yields $\approx 2 \lambda^{3} v^{3} / A_{\lambda} \sin 2 \beta \cos ^{2} 2 \beta$ in $1-3(3-1)$ element. The mass of the light doublet and that of the singlet are order of $\lambda v$. Then the mixing angle between the light doublet and the singlet is order of $\lambda v / A_{\lambda} / \tan \beta$. Similar discussion applies for the D-term potential. The diagonalization matrix $\mathcal{S}$ of $\mathcal{M}_{S}^{2}$ is given by

$$
\begin{aligned}
\mathcal{S M}_{S}^{2} \mathcal{S}^{\dagger}= & \left(\begin{array}{ccc}
m_{Z}^{2} \cos ^{2} 2 \beta+\lambda^{2} v^{2} \sin ^{2} 2 \beta & 0 & 0 \\
0 & A_{\lambda}^{2}+\left(m_{Z}^{2}-\lambda^{2} v^{2}\right) \sin ^{2} 2 \beta & 0 \\
0 & 0 & \lambda^{2} v^{2} \sin ^{2} 2 \beta
\end{array}\right) \\
& +\mathcal{O}\left(\frac{v}{A_{\lambda}}\right)^{4}, \\
\mathcal{S}^{\dagger}= & \left(\begin{array}{ccc}
\cos \beta & -\sin \beta & \lambda \frac{v}{A_{\lambda}} \sin \beta \\
\sin \beta & \cos \beta & \lambda \frac{v}{A_{\lambda}} \cos \beta \\
-\lambda \frac{v}{A_{\lambda}} \sin 2 \beta & -\lambda \frac{v}{A_{\lambda}} \cos 2 \beta & 1
\end{array}\right)+\mathcal{O}\left(\frac{v}{A_{\lambda}}\right)^{2}
\end{aligned}
$$

at tree level. Note that the mass of the light doublet originates in the symmetry breaking terms. Non-vanishing $\kappa$ and $m_{S}^{2}$ are expected to add corrections of order $\kappa$ and $m_{S}^{2} / \lambda^{2} v^{2}$ to $\mathcal{S}$. Radiative corrections to the Higgs potential are another source of the singlet-doublet mixing because the kinetic terms and the quark-lepton Yukawa couplings also break the scaling symmetry. Actually, this is not the end of story. The Higgs action has another approximate scale symmetry if $\kappa=0$ and $m_{H_{u}}^{2}=0$, in which the transformations of $H_{u}$ 
and $S$ are interchanged in eq. (4.12). The VEVs of $S$ and $H_{d}$ spontaneously break this symmetry and the Nambu-Goldstone boson appears, which consists of a combination of $S$ and $H_{d}$ corresponding to the singlet-like Higgs boson. In the vanishing $\kappa, m_{S, H_{u}}^{2},\left\langle H_{d}\right\rangle$ limit, the real parts of $S$ and $H_{u}$ are the Nambu-Goldstone bosons (mass eigenstates). After we turn on the symmetry breaking terms, the 1-3 and 3-2 elements of the mixing matrix $\mathcal{S}$ must break the both symmetries because $S$ or $H_{u}$ is still the Nambu-Goldstone boson if one of the symmetries is preserved. In addition, the mixing between $S$ and $H_{u}$ must vanish in the Lagrangian, eq. (3.3) if $H_{d}$ decouples [101]. Therefore the leading contributions to $\left(\mathcal{S}^{\dagger}\right)_{31},\left(\mathcal{S}^{\dagger}\right)_{23}$ are given by $\kappa\langle S\rangle / M_{0},\left\langle H_{d}\right\rangle / M_{0},\left\langle H_{u}\right\rangle\langle S\rangle / M_{0}^{2},\left\langle H_{u}\right\rangle m_{H_{u}}^{2} / M_{0}^{3},\langle S\rangle m_{S}^{2} / M_{0}^{3}$ and $m_{H_{u}}^{2} m_{S}^{2} / M_{0}^{4}$ at tree level. Our argument on the suppression of the singlet-doublet mixing generally holds for models with small $\kappa$ and $m_{S, H_{u}}^{2} \ll m_{H_{d}}^{2}$.

The CP-odd part of the mass matrix is diagonalized as

$$
\begin{aligned}
\mathcal{M}_{P}^{2} & =\frac{\partial^{2} V}{\partial \eta_{i} \partial \eta_{j}}=\mathcal{P}^{\dagger}\left(\begin{array}{ccc}
0 & 0 & 0 \\
0 & A_{\lambda}^{2}+\lambda^{2} v^{2} & 0 \\
0 & 0 & 0
\end{array}\right) \mathcal{P}, \\
\mathcal{P}^{\dagger} & =\left(\begin{array}{ccc}
\cos \beta & \sin \beta \cos \gamma & \sin \beta \sin \gamma \\
-\sin \beta & \cos \beta \cos \gamma & \cos \beta \sin \gamma \\
0 & -\sin \gamma & \cos \gamma
\end{array}\right),
\end{aligned}
$$

in the $\kappa=0$ limit. Here $\eta_{i}=\sqrt{2}\left(\operatorname{Im}\left(H_{d}\right), \operatorname{Im}\left(H_{u}\right), \operatorname{Im}(S)\right)$ and $\tan \gamma=\lambda v / A_{\lambda}$. Two Nambu-Goldstone bosons appear for the broken electroweak and Pecci-Quinn symmetries. The former is absorbed by $Z$ boson and the latter acquires mass with non-vanishing $\kappa$. In the following section, we show numerically the spectrum of our model.

\section{Spectrum and phenomenological aspects}

Here, we study numerically the spectrum and phenomenological aspects of our model. Our numerical calculation is performed with NMSSMTools package [143-145]. We choose input parameters as the squark and slepton masses, their tri-linear couplings, and parameters in the Higgs sector $\left(\lambda, \kappa, A_{\lambda}, A_{\kappa}, m_{H_{d}}\right.$ and $\left.\tan \beta\right)$. We first minimize the one-loop effective potential of our model at the SUSY scale and calculate the effective $\mu$-term. Then use it as an input of the NMSSMTools instead of $m_{H_{d}}$. NMSSMTools allows various levels of sophistication in the calculation of the Higgs boson mass. To keep the consistency with the input, we choose the default option (BLOCK MODSEL 8 0) which corresponds to the one-loop calculation at SUSY scale. The small parameters $m_{S}^{2}, m_{H_{u}}^{2}$ are calculated so that we can obtain the correct EW symmetry breaking. In the following, we only adopt the parameters which predict $\left|m_{S}^{2}\right|,\left|m_{H_{u}}^{2}\right| \lesssim M_{0}^{2} / 8 \pi^{2}$ within the range of the model ambiguity. We choose the small parameter $A_{\kappa}$ as an input because the number of minimization conditions is not enough to predict it. 


\subsection{Higgs mass spectrum}

We first discuss the mass spectrum of the Higgs sector. As described in the previous section, our model is in the decoupling region where the heavy doublet $\cos \beta H_{u}-\sin \beta H_{d}^{*}$ orthogonal to the flat direction has a mass much larger than the EW scale. Then this heavy doublet approximately consists of the heaviest CP-even, CP-odd and Charged Higgs bosons and they have degenerate masses $\approx M_{0}$ up to the effect of the EW symmetry breaking. Remaining light degrees of freedom are the first and second lightest CP-even Higgs bosons and the lightest CP-odd Higgs boson.

In figure 1, we show the mass spectrum of the light CP-even Higgs bosons as a function of $\lambda$ and $\kappa$ for $\tan \beta=3$ and $M_{0}=1500 \mathrm{GeV}$. In addition we present $m_{H_{u}}^{2}, m_{S}^{2}$ and $\mu$ to check whether the parameters fit in the range favored by the mirage mediation or not. We also plot the coupling squared of the lightest CP-even Higgs boson to the gauge boson normalized with its SM value. This measures the amount of the doublet component in the lightest CP-even Higgs boson. The red curve in the figure indicates the boundary where $\lambda$ and $\kappa$ blow up at the Planck scale. Inside this curve the model remains perturbative until the Planck scale. The gray shaded area around the red curve shows the region where our iterative method fails to calculate the minimum of the effective potential. This is due to the tachyonic tree-level Higgs mass which we use as the initial condition of the iteration. The quantum correction may lift it, however, we do not pursue this possibility because the region is already excluded by the LEP Higgs mass limit. The gray shaded area in the small $\kappa$ shows the region where the lightest CP-even Higgs becomes tachyonic. The yellow region is disfavored due to the false vacuum developed far beyond the EW scale as studied in $[146,147]$.

As we see in the upper left panel, the lightest CP-even Higgs boson mass can reach $125 \mathrm{GeV}$ for large value of $\lambda(\approx 0.7)$ and small value of $\kappa(\approx 0.1)$. This region is favored by the mirage mediation as shown in the middle left and middle right panels where the corresponding small mass parameters satisfy $m_{s}^{2}, m_{H_{u}}^{2} \lesssim M_{0}^{2} / 8 \pi^{2}$ for the observed Higgs boson mass. The lower right panel also indicates $h_{1}$ is almost doublet in this region. This is because the mixing between the doublet and the singlet in the mass matrix reduces the smaller eigenvalue while raises the larger eigenvalue [100-113]. Then the mass of the lightest Higgs boson is maximized when the mixing is minimized. The second lightest CP-even Higgs is around $200 \mathrm{GeV}$ in the upper right panel and dominantly consists of the singlet. The higgsino mass parameter $\mu$ is around $500 \mathrm{GeV}$ in the lower left panel which is much heavier than the EW scale, however, the cancellation mechanism described in the previous section protects the model from the fine-tuning which we will see numerically later. It is noted that the region is naturally realized in the model where $\lambda$ has a strong dynamics origin at the Planck scale and an approximate Pecci-Quinn (PQ) symmetry moderately suppresses $\kappa$.

In figure 2 , we plot the same observables for $\tan \beta=10$. The other input parameters are fixed as in figure 1. Now the lightest CP-even Higgs boson mass cannot reach $125 \mathrm{GeV}$. Instead, the second lightest CP-even Higgs boson can be as light as $125 \mathrm{GeV}$. This region is favored by the mirage mediation according to the contours of the small parameters, $m_{H_{u}}$ and $m_{S}$. In this region, the coupling squared to the gauge boson indicates that $h_{1}$ almost consists of the singlet and hence $h_{2}$ is almost doublet. Now the mixing between the doublet 

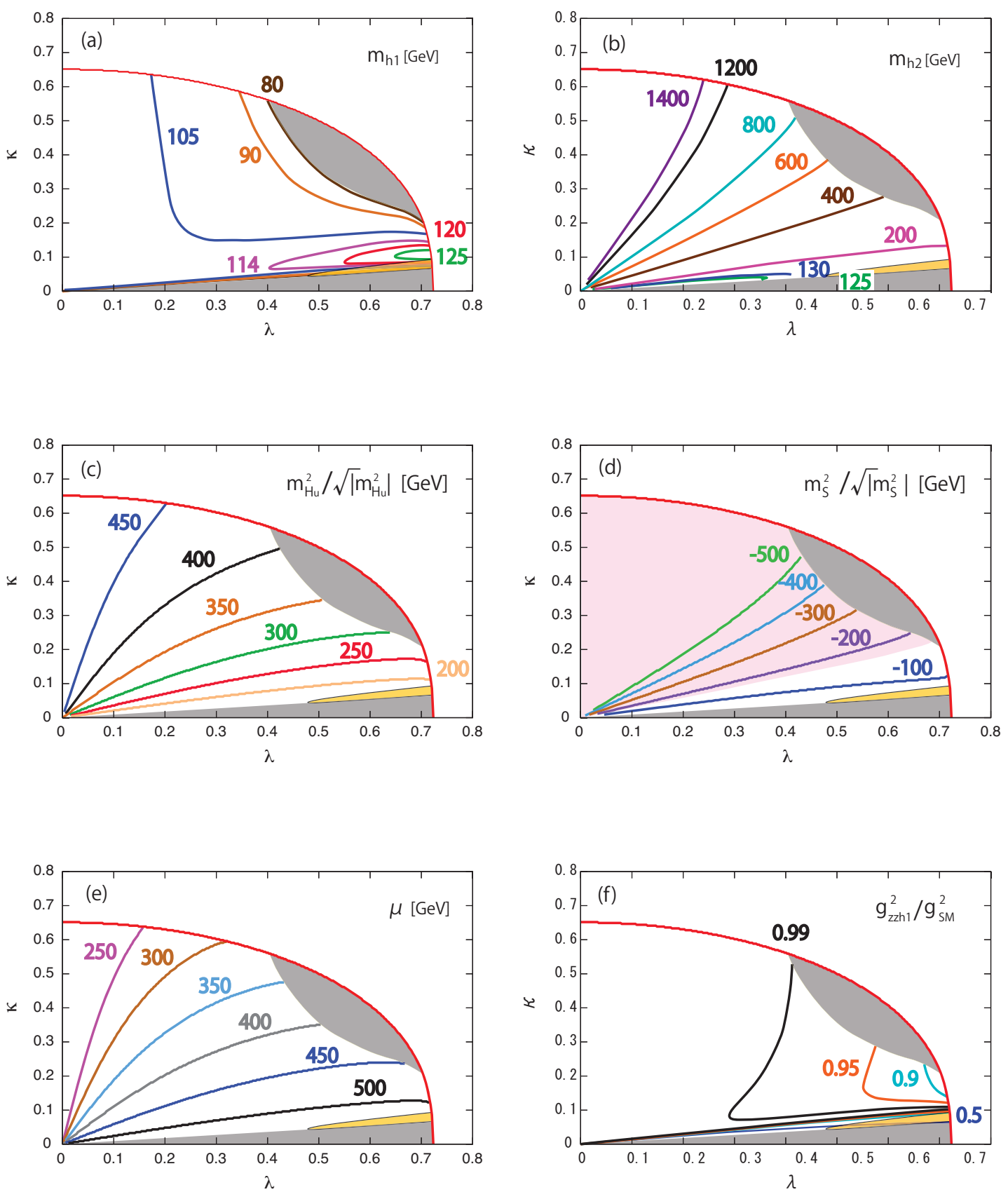

Figure 1. The CP-even Higgs masses and the Higgs mass parameters for $\tan \beta=3, M_{0}=$ $1500 \mathrm{GeV}, A_{\kappa}=-100 \mathrm{GeV}$. 

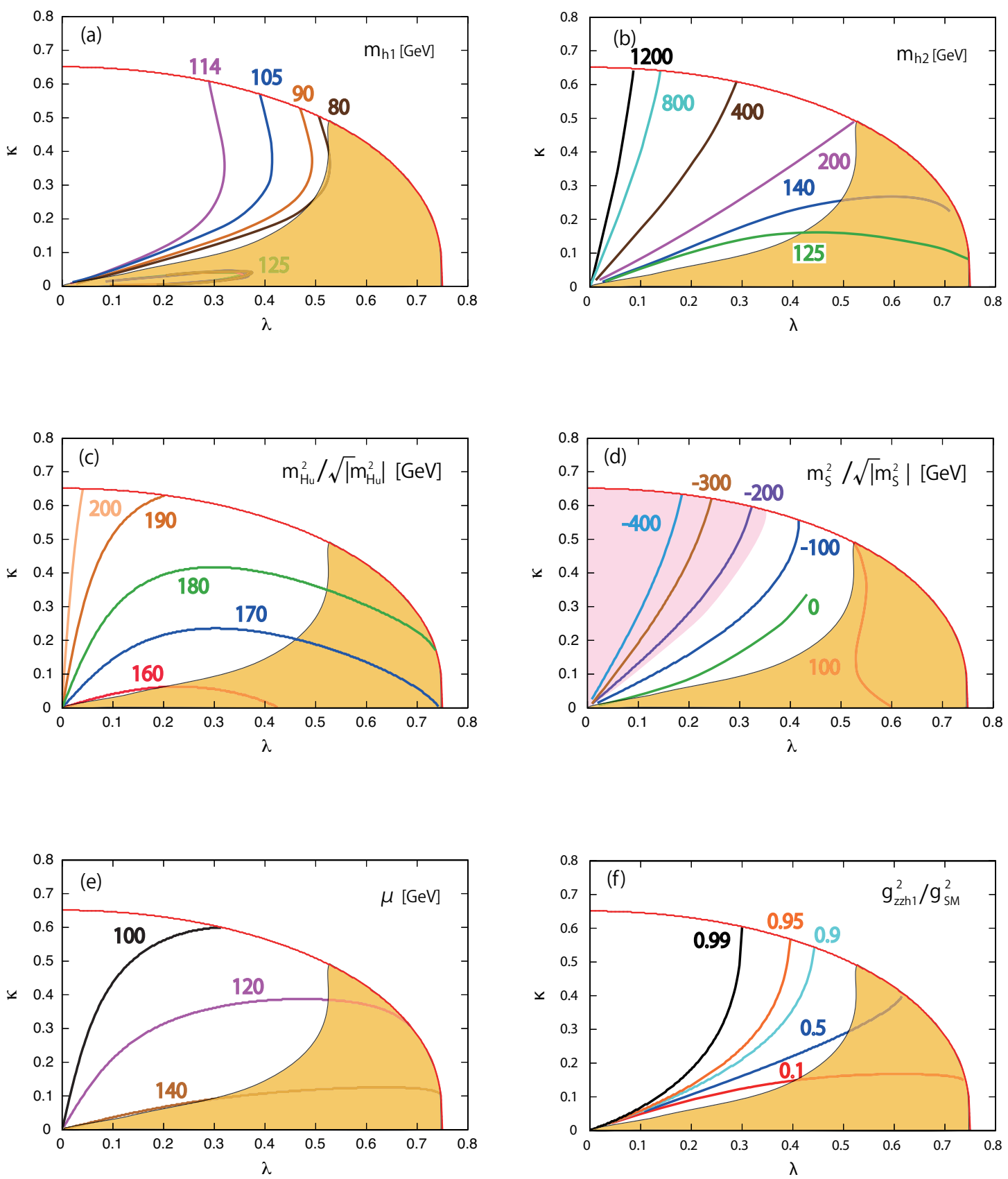

Figure 2. The CP-even Higgs mass and the Higgs mass parameters for $\tan \beta=10, M_{0}=1500 \mathrm{GeV}$, $A_{\kappa}=-100 \mathrm{GeV}$. 

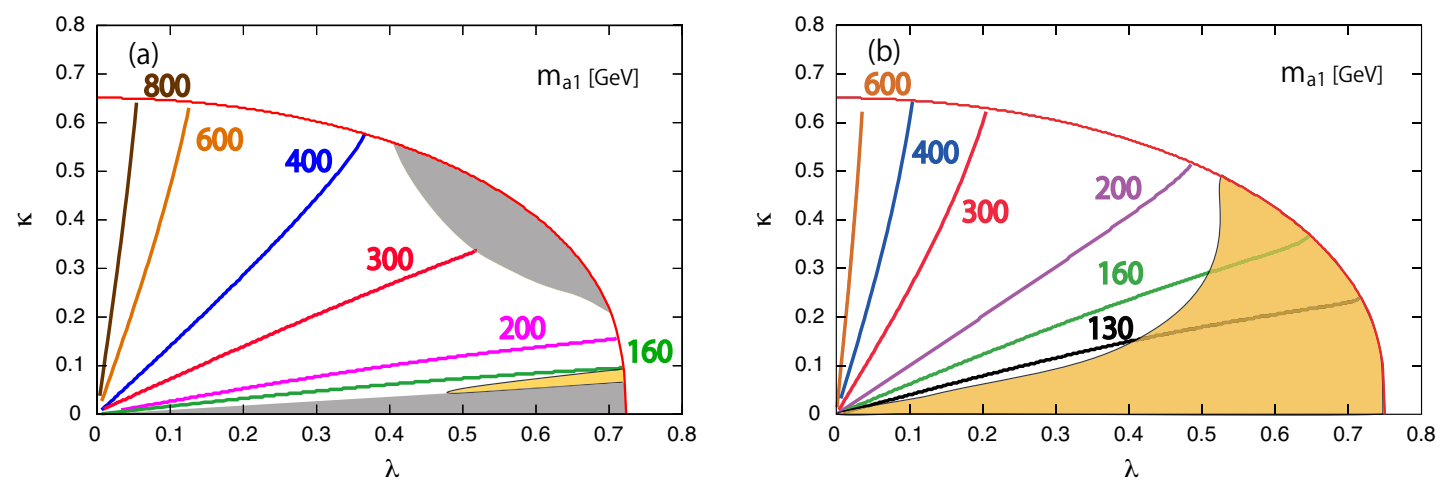

Figure 3. The lightest CP-odd Higgs mass for $\tan \beta=3$ (left) and $\tan \beta=10$ (right). The other input parameters are same as figure 1.

and the singlet in the mass matrix is non-negligible because the radiative correction is not enough to push the doublet to $125 \mathrm{GeV}$ and the mixing is required to raise the larger eigenvalue. The F-term contribution to the doublet mass from $\lambda$ is suppressed due to the large $\tan \beta$. This mixing imposes a rather strong constraint on the model from the LEP Higgs boson search via $e^{+} e^{-} \rightarrow Z^{*} \rightarrow Z h$. The region around $m_{h_{1}} \gtrsim 100 \mathrm{GeV}$ still survives and is an interesting possibility in light of the observed excess around $98 \mathrm{GeV}[99,103,125-$ 130]. If we raise the SUSY scale, the radiative correction takes the place of the mixing in realizing the $125 \mathrm{GeV}$ Higgs boson mass and the constraint is relaxed. The higgsino mass is around $M_{0} / \tan \beta \approx 150 \mathrm{GeV}$ as expected. The LEP chargino mass bound implies $\tan \beta$ cannot exceed $\approx M_{0} /(100 \mathrm{GeV})$.

For completeness, we present the contours for the lightest CP-odd Higgs boson mass in figure 3. The mass decreases as $\kappa$ is reduced as expected from the approximated PQ symmetry. The contours are almost similar to those of the second lightest CP-even Higgs boson. $125 \mathrm{GeV}$ CP-even Higgs boson corresponds to $m_{a_{1}} \simeq 200 \mathrm{GeV}$ for $\tan \beta=3$ and $m_{a_{1}} \simeq 150 \mathrm{GeV}$ for $\tan \beta=10$.

\subsection{Fine-tuning in the electroweak symmetry breaking}

Here we numerically estimate the degree of fine-tuning of the EW symmetry breaking in our model. Following the standard lore [148-160], we define the fine-tuning measure of a observable $y$ against an input parameter $x$ as

$$
\Delta_{x}^{y}=\frac{\partial \ln (y)}{\partial \ln (x)} .
$$

To evaluate the EW symmetry breaking, the simplest choice of $y$ 's are the three Higgs VEVs $v_{i}=\left(\left\langle H_{u}\right\rangle,\left\langle H_{d}\right\rangle,\langle S\rangle\right)$. Instead of them, we choose $m_{Z}^{2}, \tan \beta$ and $\mu$, extending the standard choice, $y=m_{Z}^{2}$ in the literature $[131,132,161-163]$. Here we emphasize that in principle all the VEVs must be examined to estimate the degree of fine-tuning once we extend the EW sector of the SM model, including usually disregarded $\tan \beta$. The maximum of these measures, $\Delta=\max \left|\Delta_{x}^{y}\right|$ could be regarded as the measure of the EW fine-tuning in the model. As $x$, we take $\lambda, \kappa$ and the small parameters, $m_{H_{u}}, m_{S}$ and $A_{\kappa}$ at the SUSY 
scale. Note that the large parameters such as $m_{H_{d}}, A_{\lambda}$ are not free parameters in our model and fixed by the ultraviolet physics.

These measures are easily calculated by the potential minimization conditions eq. (3.5) [156]. If we vary the input parameters $x_{a}$ with $\Delta x_{a}$, the Higgs VEVs $v_{i}$ shift with $\Delta v_{i}$ following the potential minimum and they are related as

$$
\sum_{k} \frac{\partial^{2} V}{\partial \phi_{i} \partial \phi_{k}}\left(\sqrt{2} \Delta v_{k}\right)+\sum_{a} \frac{\partial^{2} V}{\partial \phi_{i} \partial x_{a}} \Delta x_{a}=0 .
$$

Noticing that the coefficient of the first term is given by the CP even Higgs mass matrix defined in eq. (4.16), this leads to

$$
\frac{\partial v_{i}}{\partial \ln x_{a}}=-\sum_{k} \frac{1}{\sqrt{2}}\left(\mathcal{M}_{S}^{2}\right)_{i k}^{-1} \frac{\partial^{2} V}{\partial \phi_{k} \partial \ln x_{a}} .
$$

The fine-tuning measures can be constructed from these derivatives as

$$
\begin{aligned}
\Delta_{x}^{m_{Z}^{2}} & =\frac{2}{v}\left(\cos \beta \frac{\partial v_{d}}{\partial \ln x}+\sin \beta \frac{\partial v_{u}}{\partial \ln x}\right), \\
\Delta_{x}^{\tan \beta} & =\frac{1}{v_{u}} \frac{\partial v_{u}}{\partial \ln x}-\frac{1}{v_{d}} \frac{\partial v_{d}}{\partial \ln x} \\
\Delta_{x}^{\mu} & =\frac{\lambda}{\mu} \frac{\partial v_{s}}{\partial \ln x}+\delta_{x \lambda} .
\end{aligned}
$$

This expression has clear physical meaning. ${ }^{7}$ It is obvious that the measures increase if a certain Higgs mass is much lighter than the mass parameters in the potential. In NMSSMTools, the Higgs masses are calculated including the quantum corrections, however, the fine-tuning measures are calculated using the tree-level formula. This overestimates the fine-tuning if any of the tree-level masses vanish although the quantum corrections lift them. We calculate the measure using the above formula with the tree-level potential $V$ and the quantum corrected mass matrix $\mathcal{M}_{S}^{2}$.

In figure 4 , we show the fine-tuning measures, $\Delta_{x}^{m_{Z}^{2}}$ for $\tan \beta=3, M_{0}=1500 \mathrm{GeV}$, $A_{\kappa}=-100 \mathrm{GeV}$. The red dots satisfy the Higgs mass condition, $124 \mathrm{GeV}<m_{h_{1}}<$ $126 \mathrm{GeV}$. We also plot blue dots representing the region where $124 \mathrm{GeV}<m_{h_{2}}<126 \mathrm{GeV}$. Both of them are favored by the mirage mediation within the 1-loop uncertainty. Here we choose the width, $125 \pm 1 \mathrm{GeV}$ as a guide to the eye. Note that currently the theoretical error of the SM-like Higgs mass in the NMSSM could reach $5 \mathrm{GeV}$ [133] and actual bound is somewhat weaker than the bands shown in the figure. ${ }^{8}$ The upper left and upper right panels show the case for $\lambda$ and $\kappa$, respectively. Because the effective $\mu$-term is roughly proportional to $\kappa / \lambda$ as seen in the figure 2 , severe fine-tuning is expected for these parameters. However, $\Delta_{\lambda}^{m_{Z}^{2}} \lesssim 5$ and $\Delta_{\kappa}^{m_{Z}^{2}} \lesssim 4$ are realized for $m_{h_{1}} \approx 125 \mathrm{GeV}$ in our model. Also $\Delta_{\lambda, \kappa}^{m_{Z}^{2}} \lesssim 10$ is satisfied for $m_{h_{2}} \approx 125 \mathrm{GeV}$ case unless $m_{h_{1}}$ is not too light. This is not trivial because the khaki circles which do not necessarily satisfy the conditions required by

\footnotetext{
${ }^{7}$ Essentially the same formula for $y=m_{Z}^{2}$ but different presentation is given in the appendix C of [110].

${ }^{8}$ We thank F. Staub for pointing out this issue.
} 

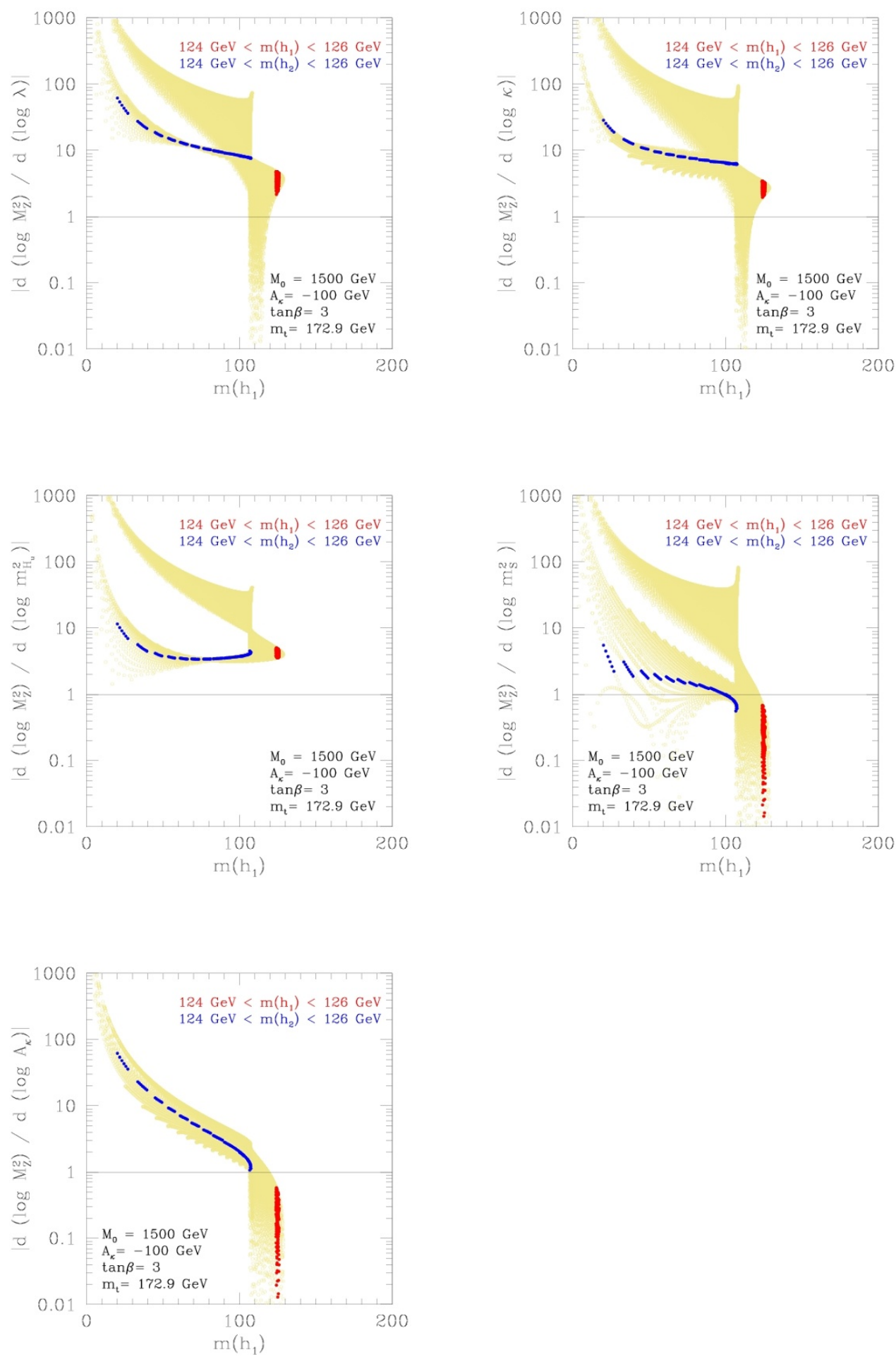

Figure 4. The fine-tuning measures of $m_{Z}^{2}$ for $\tan \beta=3, M_{0}=1500 \mathrm{GeV}, A_{\kappa}=-100 \mathrm{GeV} . \lambda$ and $\kappa$ are scanned over the white and yellow regions in figure 1 . 

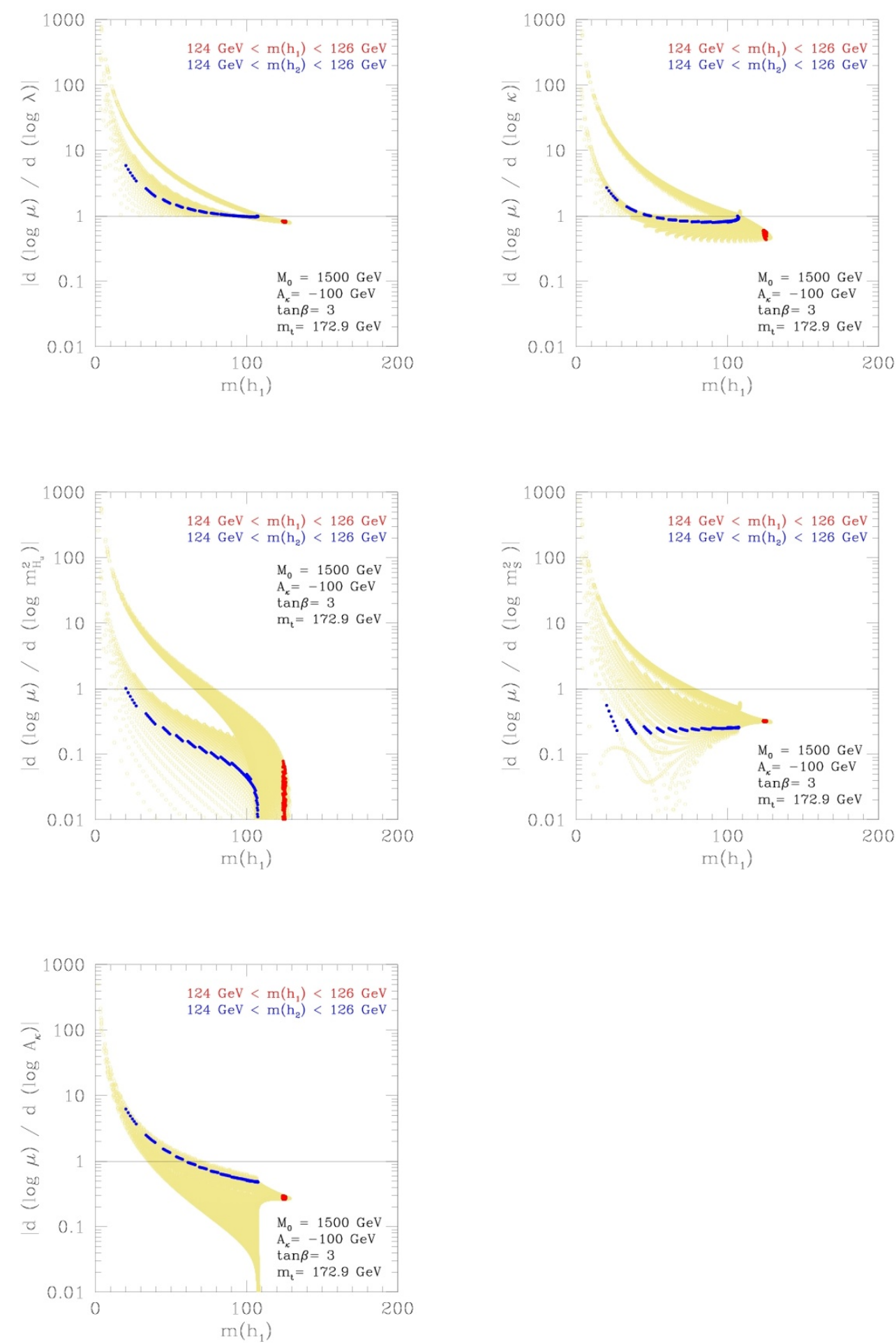

Figure 5. The fine-tuning measures of $\mu$ for $\tan \beta=3, M_{0}=1500 \mathrm{GeV}, A_{\kappa}=-100 \mathrm{GeV}$. 

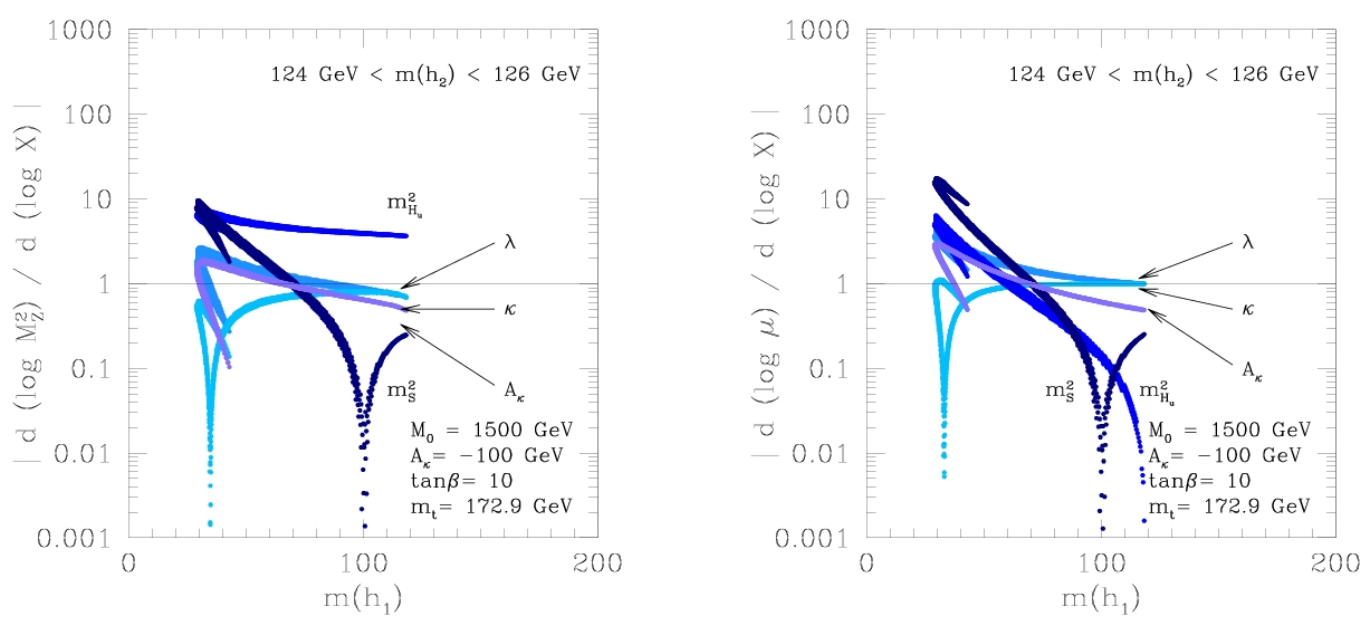

Figure 6. The fine-tuning measures of the EW symmetry breaking for $\tan \beta=10, M_{0}=1500 \mathrm{GeV}$, $A_{\kappa}=-100 \mathrm{GeV} . \lambda$ and $\kappa$ are scanned over the white and yellow regions in figure 2 . Only the points satisfying $124 \mathrm{GeV}<m_{h_{2}}<126 \mathrm{GeV}$ are depicted.

the mirage mediation reach $\Delta_{\lambda, \kappa}^{m_{Z}^{2}} \sim 100$ even with a moderate value of $m_{h_{1}}$. We confirmed that the fine-tuning measures for these dimensionless parameters do not deteriorate even if we increase $M_{0}$ to $5 \mathrm{TeV}$. The middle left and middle right panels present the results for $m_{H_{u}}^{2}$ and $m_{S}^{2}$. It is found that $\Delta_{m_{H_{u}}^{2}}^{m_{2}^{2}} \simeq 5$ and $\Delta_{m_{S}^{2}}^{m_{Z}^{2}} \lesssim 1$ for the observed Higgs mass and a moderate value of $m_{h_{1}}$. Again, this is not trivial because the khaki circles spread over $\Delta_{m_{H_{u}}^{2}, m_{S}^{2}}^{m_{S}^{2}} \sim 100$. These measures for the dimensionful parameters are sensitive to the SUSY scale, $M_{0}$. The lower left panel shows the case for $A_{\kappa}$ and we find $\Delta_{A_{\kappa}}^{m_{Z}^{2}} \lesssim 0.4$ for $m_{h_{1}} \approx 125 \mathrm{GeV}$ and $\Delta_{A_{\kappa}}^{m_{Z}^{2}} \lesssim 10$ for $m_{h_{2}} \approx 125 \mathrm{GeV}$ with a moderate value of $m_{h_{1}}$. This measure appears to be not sensitive to the SUSY scale, $M_{0}$.

In figure 5 , we show the fine-tuning measures for $\mu$ with the same input parameters. All of them are smaller than $\mathcal{O}(1)$ for the observed Higgs mass. On the other hand, $\Delta_{x}^{\tan \beta} \approx-\Delta_{x}^{\mu}$ holds well in our model because of the relation eq. (4.8a). Then $\Delta_{x}^{\tan \beta}$ do not work as independent measures. Thus the worst measure is $\Delta_{\lambda}^{m_{Z}}$, which is below 10 unless $h_{1}$ is extremely light (such a region is actually excluded by phenomenological constraints). Therefore the model requires at most $10 \%$ tuning $\left(\Delta^{-1}=0.1\right)$ for $\tan \beta=3$ and $M_{0}=1500 \mathrm{GeV}$. In our model the stop mass is $\sqrt{c_{t_{L, R}}} M_{0} \approx 1 \mathrm{TeV}$. Thus we numerically confirmed the model exhibits surprisingly low level of fine-tuning compared to the conventional SUSY breaking models.

In figure 6 , we summarize the same fine-tuning measures for the $\tan \beta=10$ case. The other input parameters are same as in figure 4 . Here, we impose $124 \mathrm{GeV}<m_{h_{2}}<$ $126 \mathrm{GeV}$. There is no parameter region satisfying $m_{h_{1}} \approx 125 \mathrm{GeV}$ in this case. The finetuning measures for $\lambda, \kappa$ and $A_{\kappa}$ are below $\mathcal{O}(1)$. The worst tuning $(\Delta)$ is $\Delta_{m_{H_{u}}^{2}}^{m_{2}^{2}} \approx 5$ for $m_{h_{1}} \gtrsim 50 \mathrm{GeV}$. While $\Delta_{m_{S}^{2}}^{m_{Z}^{2}}, \Delta_{m_{H_{u}}^{2}}^{\mu}$ and $\Delta_{m_{S}^{2}}^{\mu}$ are well below the unity with $m_{h_{1}} \sim 100 \mathrm{GeV}$, 

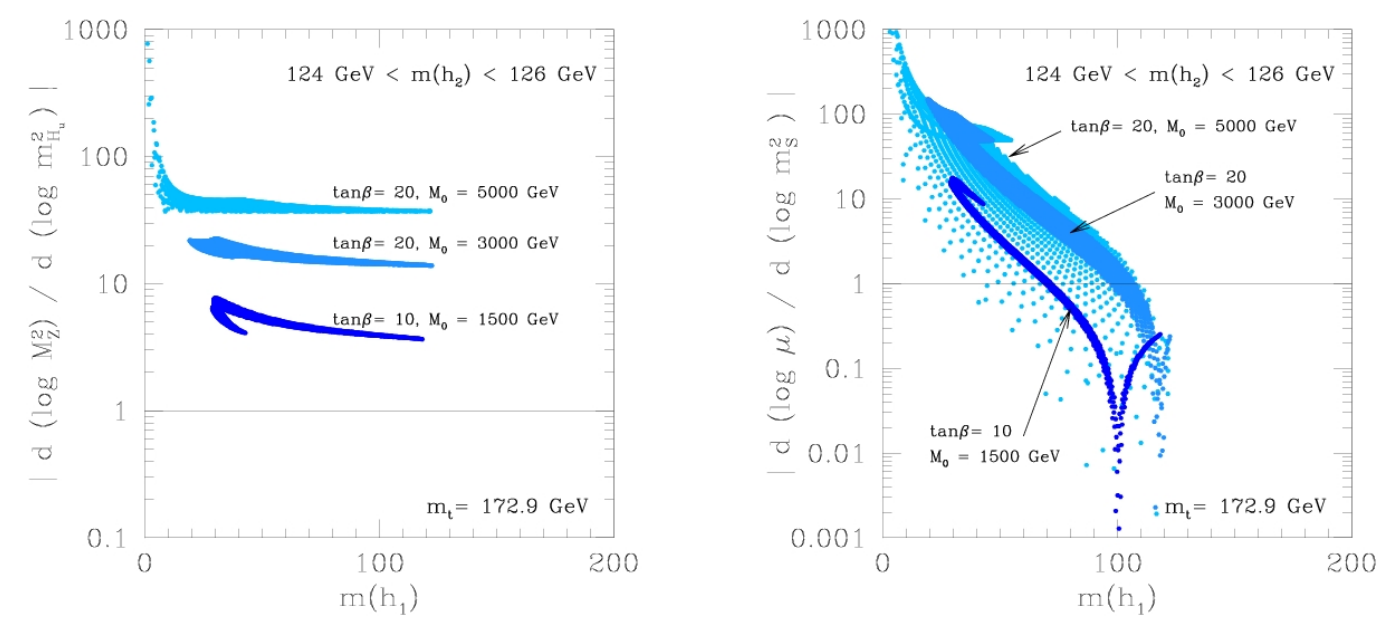

Figure 7. The fine-tuning measures $\Delta_{m_{H}^{2}}^{m_{Z}^{2}}$ (left) and $\Delta_{m_{S}^{2}}^{\mu}$ (right) for $M_{0}=3 \mathrm{TeV}$ and $M_{0}=5 \mathrm{TeV}$ $(\tan \beta=20)$ compared with $M_{0}=1.5 \mathrm{TeV}(\tan \beta=10)$ case. $A_{\kappa}$ is fixed at $A_{\kappa}=-100 \mathrm{GeV}$.

they increase up to $10-20$ once $m_{h_{1}}$ decreases down to $40 \mathrm{GeV}$. Here we do not impose any experimental constraints, however, it is interesting that in fact only the points $m_{h_{1}} \gtrsim$ $90 \mathrm{GeV}$ can survive the LEP Higgs bound with the adopted choice of parameters. This is because the mixing with the doublet of order $10 \%$ is necessary for $m_{h_{2}}$ to reach $125 \mathrm{GeV}$. This region exactly matches with the area where the tuning is minimized.

For completeness, in figure 7 we plot $\Delta_{m_{H u}^{2}}^{m_{Z}^{2}}$ and $\Delta_{m_{S}^{2}}^{\mu}$ for $M_{0}=3 \mathrm{TeV}$ and $M_{0}=5 \mathrm{TeV}$ in addition to the $M_{0}=1.5 \mathrm{TeV}$ case. The other parameters are fixed at $\tan \beta=20$ and $A_{\kappa}=-100 \mathrm{GeV}$. We see that $\Delta_{m_{H_{u}}^{2}}^{m_{Z}^{2}}$ is $10-20$ for $M_{0}=3 \mathrm{TeV}$ and $\sim 40$ for $M_{0}=5 \mathrm{TeV}$. Also we see fine-tuning measure $\Delta_{m_{S}^{2}}^{\mu} \lesssim 10$ if $m_{h_{1}} \gtrsim 60 \mathrm{GeV}$. It is remarkable that even the $5 \mathrm{TeV}$ case is acceptable in the standard of the conventional models build around $1 \mathrm{TeV}$. Again the LEP bound for $h_{1}$ is weak where the fine-tuning measures are minimized $\left(m_{h_{1}} \sim 100 \mathrm{GeV}\right)$.

\subsection{Higgs couplings and collider signature}

In this section, we discuss the couplings of the Higgs bosons to the SM particles. They play crucial roles in production and decay of the Higgs bosons at the LHC and future colliders like ILC, FCC-ee(TLEP)/CEPC. The precision measurements of the signal strength for various production and decay channels will constrain the couplings of the SM-like Higgs boson at $\mathcal{O}(1) \%$ level or below [164-169]. The pattern of deviations from the SM predictions will bring hints for underlying models beyond the SM. Also there are chances to detect extra light Higgs bosons if their couplings to the SM particles are strong enough.

The interaction of the CP-even Higgs bosons $h_{i}(i=1-3)$ with the SM fields can be described by the following effective Lagrangian [170]:

$$
\mathcal{L}=\sum_{i=1}^{3}\left[C_{V}^{i} \frac{\sqrt{2} m_{W}^{2}}{v} h_{i} W_{\mu}^{+} W^{-\mu}+C_{V}^{i} \frac{m_{Z}^{2}}{\sqrt{2} v} h_{i} Z_{\mu} Z^{\mu}-\sum_{f} C_{f}^{i} \frac{m_{f}}{\sqrt{2} v} h_{i} \bar{f} f\right.
$$




\begin{tabular}{|c|ccc|}
\hline & $h$ & $H$ & $h_{S}$ \\
\hline$m_{h_{i}}^{2}$ & $m_{Z}^{2} \cos ^{2} 2 \beta+\lambda^{2} v^{2} \sin ^{2} 2 \beta$ & $A_{\lambda}^{2}+\left(m_{Z}^{2}-\lambda^{2} v^{2}\right) \sin ^{2} 2 \beta$ & $\lambda^{2} v^{2} \sin ^{2} 2 \beta$ \\
$C_{V}^{i}$ & 1 & 0 & $\frac{\lambda v}{A_{\lambda}} \sin 2 \beta$ \\
$C_{d, e}^{i}$ & 1 & $-\tan \beta$ & $\frac{\lambda v}{A_{\lambda}} \tan \beta$ \\
$C_{u}^{i}$ & 1 & $\cot \beta$ & $\frac{\lambda v}{A_{\lambda}} \cot \beta$ \\
\hline
\end{tabular}

Table 1. Coupling constants of the CP-even Higgs bosons in the $\kappa=0, m_{S}^{2}=0$ limit.

$$
\left.+C_{g}^{i} \frac{\alpha_{s}}{12 \sqrt{2} \pi v} h_{i} G_{\mu \nu}^{a} G^{a \mu \nu}+C_{\gamma}^{i} \frac{\alpha}{\sqrt{2} \pi v} h_{i} A_{\mu \nu} A^{\mu \nu}\right],
$$

where we assume the custodial symmetry relating the $W$ and $Z$ couplings. The sum of $f$ runs all the SM fermions. $G_{\mu \nu}^{a}$ and $A_{\mu \nu}$ are the field strengths of the gluon and photon, respectively. The coupling constants in the first line are normalized so that $C_{V}^{\mathrm{SM}}=C_{f}^{\mathrm{SM}}=1$ for the SM Higgs boson $h_{\mathrm{SM}}$ at the tree level. In the NMSSM, they are given by

$$
\begin{aligned}
C_{V}^{i} & =\mathcal{S}_{2 i}^{\dagger} \sin \beta+\mathcal{S}_{1 i}^{\dagger} \cos \beta, \\
C_{f}^{i} & =\left\{\begin{array}{ll}
\mathcal{S}_{1 i}^{\dagger} \frac{1}{\cos \beta}(f=e, \mu, \tau, d, s, b) \\
\mathcal{S}_{2 i}^{\dagger} \frac{1}{\sin \beta} & (f=u, c, t)
\end{array},\right.
\end{aligned}
$$

where $\mathcal{S}_{i j}$ is the diagonalization matrix for the mass matrix of the CP-even Higgs bosons. $C_{V}^{i}$ is often quoted as $\xi_{i}$ in the literature and satisfies $\sum_{i=1}^{3} \xi_{i}^{2}=1$. We have already depicted $\xi_{1}^{2}$ in the lower right panels in figure 1 and 2. These couplings are summarized in table 1 in the $\kappa=0, m_{S}^{2}=0$ limit. The singlet-like Higgs $h_{S}$ seems to be the lightest. However, once we include corrections from non-vanishing $\kappa$ and $m_{S}^{2}$, it could be heavier than $h$ for small $\tan \beta$. The light doublet $h$ behaves like the SM Higgs boson within the approximation. Note that $C_{V}^{h}$ and $C_{u}^{h}$ are stable against the small mixing between $h$ and $H$ induced by the finite $\kappa$ and $m_{S}^{2}$, however, $C_{d, e}^{h}$ could be affected considerably by the $\tan \beta$ enhanced $C_{d, e}^{H}[107,112,171]$.

The coupling constants $C_{g}^{i}$ and $C_{\gamma}^{i}$ are loop induced. Generally, if we have a scalar $S$, a fermion $f$ and a (color-less) vector boson $\rho_{\mu}$ fields with the following couplings with the Higgs bosons [170],

$$
\mathcal{L}=\sum_{i=1}^{3}\left[-C_{s}^{i} \frac{\sqrt{2} m_{s}^{2}}{v} h_{i} S^{\dagger} S-C_{f}^{i} \frac{m_{f}}{\sqrt{s} v} h_{i} \bar{f} f+C_{\rho}^{i} \frac{\sqrt{2} m_{\rho}^{2}}{v} h_{i} \rho_{\mu}^{\dagger} \rho^{\mu}\right],
$$

their contributions to $C_{g}{ }^{i}$ and $C_{\gamma}{ }^{i}$ are given by

$$
\begin{aligned}
& \delta C_{g}^{i}=\frac{T\left(r_{s}\right)}{2} C_{s}^{i} A_{s}\left(\tau_{s}^{i}\right)+2 T\left(r_{f}\right) C_{f}^{i} A_{f}\left(\tau_{f}^{i}\right), \\
& \delta C_{\gamma}^{i}=\frac{N\left(r_{s}\right) Q_{s}^{2}}{24} C_{s}^{i} A_{s}\left(\tau_{s}^{i}\right)+\frac{N\left(r_{f}\right) Q_{f}^{2}}{6} C_{f}^{i} A_{f}\left(\tau_{f}^{i}\right)-\frac{7 Q_{\rho}^{2}}{8} C_{\rho}^{i} A_{v}\left(\tau_{\rho}^{i}\right),
\end{aligned}
$$


where $T\left(r_{s}\right)\left(T\left(r_{f}\right)\right)$ denotes the Dynkin index of the scalar (fermion) field in the representation $r_{s}\left(r_{f}\right)$ as defined by $\operatorname{tr}\left(T^{a}(r) T^{b}(r)\right)=T(r) \delta^{a b}$ with the SU(3) generator $T^{a}(r) . N\left(r_{s}\right)$ $\left(N\left(r_{f}\right)\right)$ is the number of freedom in the representation $r_{s}\left(r_{f}\right) . Q_{s, f, \rho}$ are charges of the scalar, fermion and vector fields, respectively. The mass functions $A_{s, f, v}(\tau)$ are defined as

$$
\begin{aligned}
A_{s}(\tau) & \equiv \frac{3}{\tau^{2}}[f(\tau)-\tau], \\
A_{f}(\tau) & \equiv \frac{3}{2 \tau^{2}}[(\tau-1) f(\tau)+\tau], \\
A_{v}(\tau) & \equiv \frac{1}{7 \tau^{2}}\left[3(2 \tau-1) f(\tau)+3 \tau+2 \tau^{2}\right], \\
f(\tau) & \equiv\left\{\begin{array}{cc}
\tau \leq 1 \\
-\frac{1}{4}\left[\log \frac{1+\sqrt{1-\tau^{-1}}}{1-\sqrt{1-\tau^{-1}}}-i \pi\right]^{2} \tau>1
\end{array},\right.
\end{aligned}
$$

with $\tau_{j}^{i}=\frac{m_{h_{i}}}{4 m_{j}^{2}}$. Since $A_{s, f, v}(\tau)$ decouple quickly for $\tau \gg 1$, the standard model contribution is dominated by those of the top quark and the $W$ boson and given by [170],

$$
\begin{aligned}
C_{g}^{\mathrm{SM}} & =\frac{8}{3} A_{f}\left(\tau_{t}^{\mathrm{SM}}\right) \approx 1.03 \\
C_{\gamma}^{\mathrm{SM}} & =\frac{2}{9} A_{f}\left(\tau_{t}^{\mathrm{SM}}\right)-\frac{7}{8} A_{v}\left(\tau_{W}^{\mathrm{SM}}\right) \approx-0.81 .
\end{aligned}
$$

For $\tau \ll 1$, we obtain $A_{s, f, v} \simeq 1$. Then, we have,

$$
\begin{aligned}
& \delta C_{g}^{i} \simeq \frac{T\left(r_{s}\right)}{2} C_{s}^{i}+2 T\left(r_{f}\right) C_{f}^{i}, \\
& \delta C_{\gamma}^{i} \simeq \frac{N\left(r_{s}\right) Q_{s}^{2}}{24} C_{s}^{i}+\frac{N\left(r_{f}\right) Q_{f}^{2}}{6} C_{f}^{i}-\frac{7 Q_{\rho}^{2}}{8} C_{\rho}^{i} .
\end{aligned}
$$

Together with the relation,

$$
\begin{aligned}
& C_{s}^{i} \frac{\sqrt{2} m_{s}^{2}}{v}=\frac{\partial m_{s}^{2}}{\partial h_{i}}, \quad C_{f}^{i} \frac{m_{f}}{\sqrt{2} v}=\frac{\partial m_{f}}{\partial h_{i}}, \quad C_{\rho}^{i} \frac{\sqrt{2} m_{\rho}^{2}}{v}=\frac{\partial m_{\rho}^{2}}{\partial h_{i}}, \\
\rightarrow & C_{j}^{i}=\frac{v}{\sqrt{2}} \frac{\partial \log m_{j}^{2}}{\partial h_{i}},
\end{aligned}
$$

we arrive at

$$
\begin{aligned}
\delta C_{g}^{i}=\frac{3 v}{2 \sqrt{2}} & {\left[\frac{T\left(r_{s}\right)}{3} \frac{\partial \log \operatorname{det}\left(\mathcal{M}_{s}^{2}\right)}{\partial h_{i}}+\frac{4 T\left(r_{f}\right)}{3} \frac{\partial \log \operatorname{det}\left(\mathcal{M}_{f}^{2}\right)}{\partial h_{i}}\right] } \\
\delta C_{\gamma}^{i}=\frac{v}{8 \sqrt{2}} & {\left[\frac{N\left(r_{s}\right) Q_{s}^{2}}{3} \frac{\partial \log \operatorname{det}\left(\mathcal{M}_{s}^{2}\right)}{\partial h_{i}}+\frac{4 N\left(r_{f}\right) Q_{f}^{2}}{3} \frac{\partial \log \operatorname{det}\left(\mathcal{M}_{f}^{2}\right)}{\partial h_{i}}\right.} \\
& \left.-7 Q_{\rho}^{2} \frac{\partial \log \operatorname{det}\left(\mathcal{M}_{\rho}^{2}\right)}{\partial h_{i}}\right]
\end{aligned}
$$

where $\mathcal{M}_{s, f, \rho}^{2}$ is the mass matrix for the corresponding field. Noticing that the coefficients of the derivatives are given by the steps of the beta function coefficients after integrating out 
the corresponding fields, this can be cast into the well known low energy theorem [172-176]:

$$
C_{g}^{i} \frac{\alpha_{s}}{12 \sqrt{2} \pi v}=\frac{1}{4} \frac{\partial \log \alpha_{s}(\mu)}{\partial h_{i}}, \quad C_{\gamma}^{i} \frac{\alpha}{\sqrt{2} \pi v}=\frac{1}{4} \frac{\partial \log \alpha(\mu)}{\partial h_{i}},
$$

if all the integrated fields are much heavier than the renormalization scale, $\mu$. Here, $h_{i}$ dependence of $\alpha_{s}$ and $\alpha$ enters through the mass thresholds. The contribution from the light fields should be included separately.

In SUSY models, additional contribution comes from the charged Higgs boson, sfermion and chargino loops. The charged Higgs contribution to $\delta C_{\gamma}^{h, S}$ is calculated as

$$
\delta C_{\gamma}^{i} \approx\left\{\begin{array}{cc}
\frac{1}{48}\left\{\left(-\lambda^{2}+g_{2}^{2}\right) \sin 2 \beta+\frac{1}{2}\left(g_{1}^{2}+g_{2}^{2}\right) \cos ^{2} 2 \beta\right\} \frac{v^{2}}{m_{h^{-}}^{2}} & (i=h) \\
\frac{1}{48}\left\{\left(-\lambda^{2}+g_{2}^{2}\right) \cos 2 \beta+\frac{1}{2}\left(g_{1}^{2}+g_{2}^{2}\right) \sin ^{2} 2 \beta\right\} \frac{v^{2}}{m_{h^{-}}^{2}} & (i=H) \\
\frac{1}{24}(2 \lambda+\kappa \sin 2 \beta) \frac{\mu v}{m_{h^{-}}^{2}} & (i=S) .
\end{array}\right.
$$

They are suppressed by $v^{2} / m_{h^{-}}^{2}, \mu v / m_{h^{-}}^{2}$ and negligible in our model if $m_{h^{-}}\left(\approx M_{0}\right)$ is above the $\mathrm{TeV}$ scale.

The sfermion and chargino mass matrices are given by

$$
\begin{gathered}
\mathcal{M}_{\tilde{u}}^{2}=\left(\begin{array}{cc}
m_{Q_{i}}^{2}+y_{u_{i}}^{2} v_{u}^{2}+\left(v_{u}^{2}-v_{d}^{2}\right)\left(\frac{g_{1}^{2}}{12}+\frac{g_{2}^{2}}{4}\right) & y_{u_{i}}\left(A_{u_{i}} v_{u}-\mu v_{d}\right) \\
y_{u_{i}}\left(A_{u_{i}} v_{u}-\mu v_{d}\right) & m_{\bar{U}_{i}}^{2}+y_{u_{i}}^{2} v_{u}^{2}-\left(v_{u}^{2}-v_{d}^{2}\right) \frac{g_{1}^{2}}{3}
\end{array}\right), \\
\mathcal{M}_{\tilde{d}}^{2}=\left(\begin{array}{cc}
m_{Q_{i}}^{2}+y_{d_{i}}^{2} v_{d}^{2}+\left(v_{u}^{2}-v_{d}^{2}\right)\left(\frac{g_{1}^{2}}{12}+\frac{g_{2}^{2}}{4}\right) & y_{d_{i}}\left(A_{d_{i}} v_{d}-\mu v_{u}\right) \\
y_{d_{i}}\left(A_{d_{i}} v_{d}-\mu v_{u}\right) & m_{\bar{D}_{i}}^{2}+y_{d_{i}}^{2} v_{d}^{2}+\left(v_{u}^{2}-v_{d}^{2}\right) \frac{g_{1}^{2}}{6}
\end{array}\right) \\
\mathcal{M}_{\tilde{\ell}}^{2}=\left(\begin{array}{cc}
m_{L_{i}}^{2}+y_{\ell_{i}}^{2} v_{d}^{2}+\left(v_{u}^{2}-v_{d}^{2}\right)\left(\frac{g_{1}^{2}+g_{2}^{2}}{4}\right) & y_{\ell_{i}}\left(A_{\ell_{i}} v_{d}-\mu v_{u}\right) \\
y_{\ell_{i}}\left(A_{\ell_{i}} v_{d}-\mu v_{u}\right) & m_{\bar{E}_{i}}^{2}+y_{\ell_{i}}^{2} v_{d}^{2}+\left(v_{u}^{2}-v_{d}^{2} \frac{g_{1}^{2}}{2}\right.
\end{array}\right) \\
M_{\chi^{-}}=\left(\begin{array}{cc}
M_{2} & g_{2} v_{u} \\
g_{2} v_{d} & \mu
\end{array}\right)
\end{gathered}
$$

where $v_{u, d}=\left\langle H_{u, d}\right\rangle$. The determinants of these mass matrices are calculated as

$$
\begin{aligned}
\operatorname{det}\left(\mathcal{M}_{\tilde{u}}^{2}\right)= & \prod_{i}\left[m_{Q_{i}}^{2} m_{\bar{U}_{i}}^{2}+y_{u_{i}}^{2} v_{u}^{2}\left\{m_{Q_{i}}^{2}+m_{\bar{U}_{i}}^{2}-\left(A_{u_{i}}-\mu \cot \beta\right)^{2}\right\}\right. \\
& \left.+\left(v_{u}^{2}-v_{d}^{2}\right)\left\{\left(\frac{g_{1}^{2}}{12}+\frac{g_{2}^{2}}{4}\right) m_{\bar{U}_{i}^{2}}-\frac{g_{1}^{2}}{3} m_{Q_{i}}^{2}\right\}+\mathcal{O}\left(v^{4}\right)\right], \\
\operatorname{det}\left(\mathcal{M}_{\tilde{d}}^{2}\right)= & \prod_{i}\left[m_{Q_{i}}^{2} m_{\bar{D}_{i}}^{2}+y_{d_{i}}^{2} v_{d}^{2}\left\{m_{Q_{i}}^{2}+m_{\bar{D}_{i}}^{2}-\left(A_{d_{i}}-\mu \tan \beta\right)^{2}\right\}\right. \\
& \left.+\left(v_{u}^{2}-v_{d}^{2}\right)\left\{\left(\frac{g_{1}^{2}}{12}+\frac{g_{2}^{2}}{4}\right) m_{\bar{D}_{i}^{2}}+\frac{g_{1}^{2}}{6} m_{Q_{i}}^{2}\right\}+\mathcal{O}\left(v^{4}\right)\right],
\end{aligned}
$$




$$
\begin{aligned}
\operatorname{det}\left(\mathcal{M}_{\tilde{\ell}}^{2}\right)= & \prod_{i}\left[m_{L_{i}}^{2} m_{\bar{E}_{i}}^{2}+y_{\ell_{i}}^{2} v_{d}^{2}\left\{m_{L_{i}}^{2}+m_{\bar{D}_{i}}^{2}-\left(A_{\ell_{i}}-\mu \tan \beta\right)^{2}\right\}\right. \\
& \left.+\left(v_{u}^{2}-v_{d}^{2}\right)\left\{\left(\frac{g_{1}^{2}+g_{2}^{2}}{4}\right) m_{\bar{E}_{i}^{2}}+\frac{g_{1}^{2}}{2} m_{L_{i}}^{2}\right\}+\mathcal{O}\left(v^{4}\right)\right], \\
\operatorname{det}\left(\mathcal{M}_{\chi^{-}}\right)= & M_{2} \mu-g_{2}^{2} v_{u} v_{d} .
\end{aligned}
$$

Thus the squark-slepton contributions to $C_{g}^{i}$ and $C_{\gamma}^{i}$ are suppressed by $v^{2} / m_{\tilde{q}, \tilde{\ell}}^{2}$ at leading order and almost negligible if the squarks and sleptons are above the $\mathrm{TeV}$ scale. In addition, they are further suppressed if the following conditions are satisfied by the fields coupling via non-vanishing Yukawa interactions,

$$
\begin{aligned}
& m_{Q_{i}}^{2}+m_{\bar{U}_{i}}^{2}-\left(A_{u_{i}}-\mu \cot \beta\right)^{2} \approx 0, \\
& m_{Q_{i}}^{2}+m_{\bar{D}_{i}}^{2}-\left(A_{d_{i}}-\mu \tan \beta\right)^{2} \approx 0, \\
& m_{L_{i}}^{2}+m_{\bar{E}_{i}}^{2}-\left(A_{\ell_{i}}-\mu \tan \beta\right)^{2} \approx 0 .
\end{aligned}
$$

It is interesting to observe that this is exactly the prediction of the $\mathrm{TeV}$ scale mirage mediation,

$$
\begin{array}{ll}
c_{H_{u}}=0, & c_{\tilde{t}_{L}}+c_{\tilde{t}_{R}}=1, \\
c_{H_{d}}=1, & c_{\tilde{b}_{L}}+c_{\tilde{b}_{R}}=1,
\end{array}
$$

with $\mu \approx M_{0} / \tan \beta \cdot{ }^{9}$ If any of the squarks or sleptons are light (e.g. $c_{\tilde{t}_{R}}=0$ ), this provides an exception of the anti-correlation between the fine-tuning of the EW symmetry breaking and the new physics contribution to the Higgs couplings [177].

The chargino contribution can be comparable to the SM contribution if the chargino mass is light enough $[107,170,178,179]$. In the $\kappa=0, m_{S}^{2}=0$ limit, it is calculated as

$$
\delta C_{\gamma}^{i}=\frac{v}{3 \sqrt{2}} \frac{\left(\mathcal{S}^{\dagger}\right)_{3 i} M_{2} \lambda}{M_{2} \mu-g_{2}^{2} v^{2} \cos \beta \sin \beta} \approx \frac{\lambda}{3 \sqrt{2}} \frac{v}{\mu}\left\{\begin{array}{cc}
(-\lambda) \frac{v}{A_{\lambda}} \sin 2 \beta & (i=h) \\
(-\lambda) \frac{v}{A_{\lambda}} \cos 2 \beta\left(-\frac{12}{\tan ^{2} \beta}\right) & (i=H) \\
1 & (i=S)
\end{array}\right.
$$

For $i=H$, we multiplied a suppression factor, $A_{s}(\tau) \simeq-12 / \tan ^{2} \beta$. If $\mu \sim v, \lambda \sim 1$ and once the finite $\kappa$ and $m_{S}^{2}$ induce a mixing of $\mathcal{O}(10) \%$ between the singlet and the heavy doublet, then $\mathcal{O}(10) \%$ deviation is expected in $C_{\gamma}^{h}$.

The effective Lagrangian describing the CP-odd Higgs bosons is given by

$$
\begin{aligned}
\mathcal{L}=\sum_{i=1}^{2} & {\left[i \sum_{f} C_{f}^{\prime i} \frac{m_{f}}{\sqrt{2} v} a_{i} \bar{f} \gamma_{5} f\right.} \\
& \left.+C_{g}^{\prime i} \frac{\alpha_{s}}{8 \sqrt{2} \pi v} a_{i} \epsilon^{\mu \nu \rho \sigma} G_{\mu \nu}^{a} G_{\rho \sigma}^{a}+C_{\gamma}^{\prime i} \frac{\alpha}{8 \sqrt{2} \pi v} a_{i} \epsilon^{\mu \nu \rho \sigma} A_{\mu \nu} A_{\rho \sigma}\right] .
\end{aligned}
$$

\footnotetext{
${ }^{9}$ This suppression also occurs in the type I model of [70].
} 


\begin{tabular}{|c|cc|}
\hline & $a_{1}$ & $a_{2}$ \\
\hline$m_{h_{i}}^{2}$ & 0 & $A_{\lambda}^{2}+\lambda^{2} v^{2}$ \\
$C_{d, e}^{i}$ & $\tan \beta \sin \gamma$ & $\tan \beta \cos \gamma$ \\
$C_{u}^{i}$ & $\cot \beta \sin \gamma$ & $\cot \beta \cos \gamma$ \\
\hline
\end{tabular}

Table 2. Coupling constants of the CP-odd Higgs bosons in the limit $\kappa=0$, where $\tan \gamma=\lambda v / A_{\lambda}$.

The renormalizable couplings between the CP-odd Higgs boson and the vector bosons are forbidden by the CP symmetry. We omit the loop induced couplings with $W / Z$ bosons because they do not give sizable effects in the production and decay of the Higgs bosons. The coupling constants with the fermions are given by

$$
C_{f}^{\prime i}=\left\{\begin{array}{l}
\left(\mathcal{P}^{\dagger}\right)_{1 i} \frac{1}{\cos \beta}(f=e, \mu, \tau, d, s, b) \\
\left(\mathcal{P}^{\dagger}\right)_{2 i} \frac{1}{\sin \beta} \quad(f=u, c, t)
\end{array}\right.
$$

where $\mathcal{P}$ is the diagonalization matrix for the mass matrix of the CP-odd Higgs bosons. These coupling constants in the $\kappa=0$ limit are summarized in the table 2. Note that those of $a_{1}$ are highly suppressed by $\sin \gamma \simeq \lambda v / A_{\lambda}$ because they require the mixing with the heavy doublet. The coupling constants with gluon and photon are calculated as [180-182],

$$
\begin{aligned}
\delta C_{g}^{\prime i} & =\frac{T\left(r_{f}\right)}{2} C_{f}^{\prime i} A_{f}^{\prime}\left(\tau_{f}^{i}\right), \\
\delta C_{\gamma}^{\prime} & =\frac{N\left(r_{f}\right) Q_{f}^{2}}{2} C_{f}^{\prime i} A_{f}^{\prime}\left(\tau_{f}^{i}\right),
\end{aligned}
$$

where the mass function is given by

$$
A_{f}^{\prime}(\tau) \equiv 2 \tau^{-1} f(\tau)
$$

In the limit $\tau_{f}^{i} \ll 1$, they are approximated as

$$
\begin{aligned}
& \delta C_{g}^{i} \simeq T\left(r_{f}\right) C_{f}^{\prime i}, \\
& \delta C_{\gamma}^{i} \simeq N\left(r_{f}\right) Q_{f}^{2} C_{f}^{\prime i} .
\end{aligned}
$$

The charged Higgs contribution is forbidden by the CP symmetry and the sfermion contribution is highly suppressed as in the CP-even case. The chargino contribution to $C_{\gamma}$ is approximated as

$$
\begin{aligned}
\delta C_{\gamma}^{i} & \simeq \sum_{a=1}^{2} C_{\chi_{a}^{-}}^{\prime i}=i \sqrt{2} v \frac{\partial \ln \operatorname{det}\left(\mathcal{M}_{\chi^{-}}\right)}{\partial a_{i}} \simeq-\frac{v}{\mu} \lambda\left(\mathcal{P}^{\dagger}\right)_{4-i 3} \\
& \simeq\left\{\begin{array}{cc}
-\frac{\lambda v}{\mu} & (i=1) \\
\frac{\lambda^{2} v^{2}}{\mu^{2}} \sin \beta \cos ^{2} \beta\left[-\frac{1}{\tan ^{2} \beta}\left(\ln \tan ^{2} \beta-i \pi\right)^{2}\right] & (i=2)
\end{array}\right.
\end{aligned}
$$



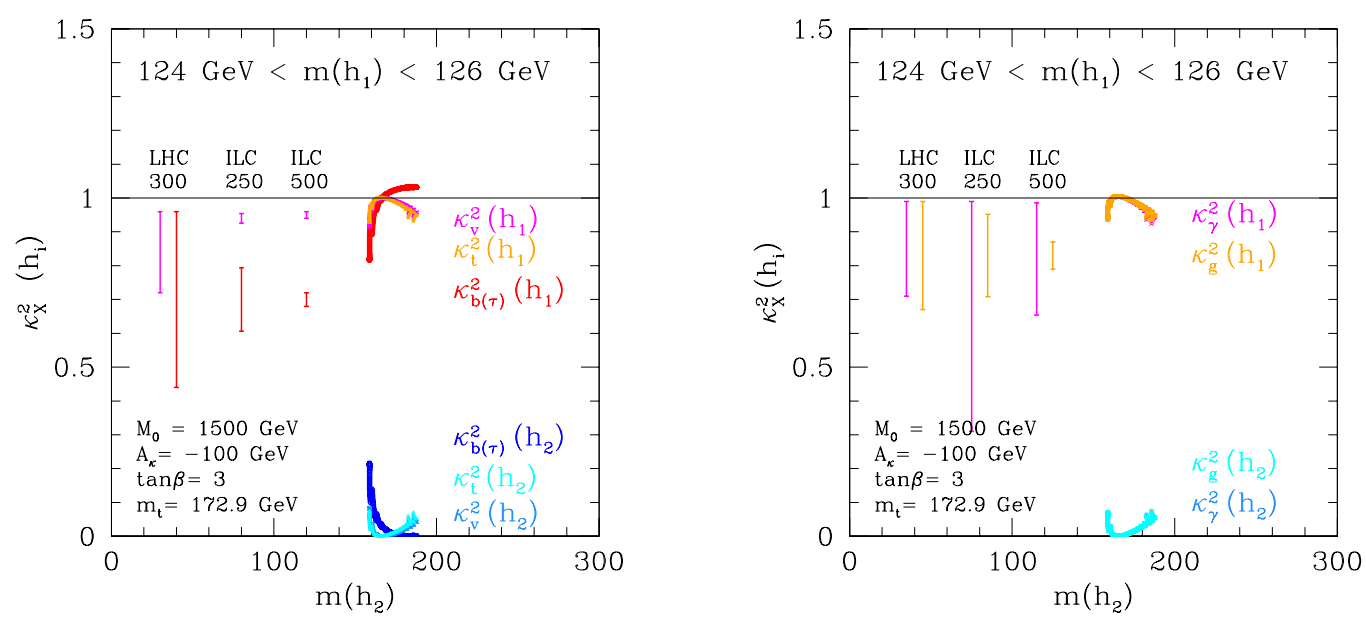

Figure 8. The scale factors of the CP-even Higgs coupling constants for $\tan \beta=3, M_{0}=1500 \mathrm{GeV}$ and $A_{\kappa}=-100 \mathrm{GeV}$. The vertical lines show the expected precisions of the SM-like Higgs coupling constants for LHC and ILC, $\Delta\left(\kappa_{X}\right)^{2} \approx 2 \Delta \kappa_{X}$.

For $i=2$, we multiplied a suppression factor, $A_{s}^{\prime}(\tau) / 2$ inside the bracket. If $\mu \sim v, \lambda \sim 1$, $C_{\gamma}^{\prime 1}$ could be comparable with $C_{\gamma}^{\mathrm{SM}}$.

Next we present the results of our numerical calculation on the coupling constants including the effect of finite $\kappa$ and $m_{S}^{2}$, the radiative corrections and various phenomenological constraints. Here we use the scale factors of the Higgs couplings relative to the SM instead of the coupling constants themselves [183, 184],

$$
\kappa_{X}^{i}=\frac{C_{X}^{i}}{C_{X}^{\mathrm{SM}}}
$$

In figure 8, we plot the $\kappa$ 's for $\tan \beta=3, M_{0}=1500 \mathrm{GeV}$ and $A_{\kappa}=-100 \mathrm{GeV}$. The horizontal axis is chosen as the mass of the singlet-like Higgs boson which is heavier than the SM-like Higgs boson for small $\tan \beta$. We impose the condition $124 \mathrm{GeV}<m_{h_{1}}<$ $126 \mathrm{GeV}$ and various phenomenological constraints built in the NMSSM Tools package. The parameter region with $124 \mathrm{GeV}<m_{h_{2}}<126 \mathrm{GeV}$ is excluded by the LEP Higgs boson search [99]. The vertical lines show the expected precision of the coupling constants for LHC $\left(300 \mathrm{fb}^{-1}\right)$ [164], ILC $(250 \mathrm{GeV})$ and ILC $(250 \mathrm{GeV}+500 \mathrm{GeV})$ [165]. (The position of the center is arbitrary.) We also quote the numbers in table 3. We plot the precision of $\kappa_{Z}^{2}$ for $\kappa_{V}^{2}$ and take conservative values for the LHC expectation.

The pseudo Nambu-Goldstone nature of the SM-like Higgs boson well suppresses the mixing with the singlet and the deviation from the SM is at most $20 \%$. This suppression is also important for not reducing the lighter SM-like Higgs boson mass due to the mixing. The approximate sum rule $\left(\kappa_{X}^{1}\right)^{2}+\left(\kappa_{X}^{2}\right)^{2}=1$ means that the deviation mainly comes from the mixing with the singlet and the SUSY contribution is negligible except for $b(\tau)$ for which the contamination of the heavy doublet has $\tan \beta$ enhanced coupling. The singletlike Higgs boson is hidden from the collider search and an interesting target for the precision measurement at the LHC and ILC. In this parameter region, the prediction does not change 


\begin{tabular}{|c|cccccc|}
\hline Facility & LHC & HL-LHC & ILC $(250)$ & ILC(500) & ILC(1000) & ILC(LumUP) \\
$\sqrt{s}(\mathrm{GeV})$ & 1,400 & 1,400 & 250 & $250+500$ & $250+500+1000$ & $250+500+1000$ \\
$\int \mathcal{L} d t\left(f b^{-1}\right)$ & $300 /$ expt & $3000 /$ expt & 250 & $250+500$ & $250+500+1000$ & $1150+1600+2500$ \\
\hline$\kappa_{\gamma}$ & $5-7 \%$ & $2-5 \%$ & $17 \%$ & $8.3 \%$ & $3.8 \%$ & $2.3 \%$ \\
$\kappa_{g}$ & $6-8 \%$ & $3-5 \%$ & $6.1 \%$ & $2.0 \%$ & $1.1 \%$ & $0.7 \%$ \\
$\kappa_{W}$ & $4-6 \%$ & $2-5 \%$ & $4.7 \%$ & $0.4 \%$ & $0.3 \%$ & $0.2 \%$ \\
$\kappa_{Z}$ & $4-6 \%$ & $2-4 \%$ & $0.7 \%$ & $0.5 \%$ & $0.5 \%$ & $0.3 \%$ \\
$\kappa_{\ell}$ & $6-8 \%$ & $2-5 \%$ & $5.2 \%$ & $1.9 \%$ & $1.3 \%$ & $0.7 \%$ \\
$\kappa_{b}$ & $10-13 \%$ & $4-7 \%$ & $4.7 \%$ & $1.0 \%$ & $0.6 \%$ & $0.4 \%$ \\
$\kappa_{t}$ & $14-15 \%$ & $7-10 \%$ & $6.4 \%$ & $2.5 \%$ & $1.3 \%$ & $0.9 \%$ \\
\hline
\end{tabular}

Table 3. Expected precisions on the Higgs coupling for LHC [164] and ILC [165]. Two numbers for LHC represent different assumptions on the systematic error. The seven parameter HXSWG benchmark parametrization $[183,184]$ is assumed.

significantly even if we adopt a conservative bound, $120 \mathrm{GeV}<m_{h_{1}}<130 \mathrm{GeV}$, taking into account the theoretical error [133].

In figure 9, we plot the same scale factors (normalized by the SM Higgs couplings) for $\tan \beta=10$. Increasing $\tan \beta$, the singlet-like Higgs boson is now lighter than the SM-like Higgs boson and we impose $124 \mathrm{GeV}<m_{h_{2}}<126 \mathrm{GeV}$. We again choose the horizontal axis as the singlet-like Higgs mass. The tree-level contribution to the SMlike Higgs mass is now not effective with larger $\tan \beta$ and small mixing with the singlet is favorable to achieve $125 \mathrm{GeV}$. However, a strong constraint exists for the light singlet from the LEP Higgs boson search if it couples with the SM particles through the mixing with the SM-like Higgs boson [99]. The khaki shaded region is mostly excluded by this constraint. The region $90 \mathrm{GeV} \lesssim m_{h_{1}} \lesssim 100 \mathrm{GeV}$ survives due to a small excess in the LEP measurement [99, 103, 125-132]. Around $m_{h_{1}} \simeq 120 \mathrm{GeV}$, the two bosons almost degenerate and the mixing is maximized, but the LEP bound is weak in this region. For large $\lambda$, the new radiative corrections to the SM-like Higgs mass, eq. (4.11) could relax the mixing with the singlet (around $m_{h_{1}} \simeq 40 \mathrm{GeV}$ ). In this parameter region, the lightest neutralino becomes lighter than the half of the $Z$ boson mass and the decay channel, $Z \rightarrow$ $2 \chi_{1}^{0}$ opens. Then the region is excluded by the measurement of the $Z$ boson width [185]. The mixing with the singlet leads to large deviations in the coupling constants of the SMlike Higgs boson observable at the LHC. On the other hand, searching the light singlet boson missed by LEP is important physics at ILC. The sum rule holds again except for $b(\tau)$ and $g$, for which the mixing with the heavy doublet is not negligible due to the $\tan \beta$ enhancement. The small excess in $\left(\kappa_{\gamma}^{2}\right)^{2}$ around $m_{h_{1}} \simeq 30 \mathrm{GeV}$ is contribution from the chargino interfering with that of the SM. The surviving region expands once we raise the SUSY breaking scale $M_{0}$ and increase the top radiative correction to the SM-like Higgs mass. Also note that the prediction is sensitive to the theoretical error in the Higgs mass calculation. If we take a conservative bound, e.g. $120 \mathrm{GeV}<m_{h_{2}}<130 \mathrm{GeV}$, much lighter $h_{1}\left(m_{h_{1}} \gtrsim 20 \mathrm{GeV}\right)$ is allowed because the singlet-doublet mixing is suppressed. For such $m_{h_{1}}$, the $h_{1}$ couplings to the SM particles and the deviations of $h_{2}$ couplings from those of the SM Higgs boson are highly suppressed except for $b$ and $\tau$. 

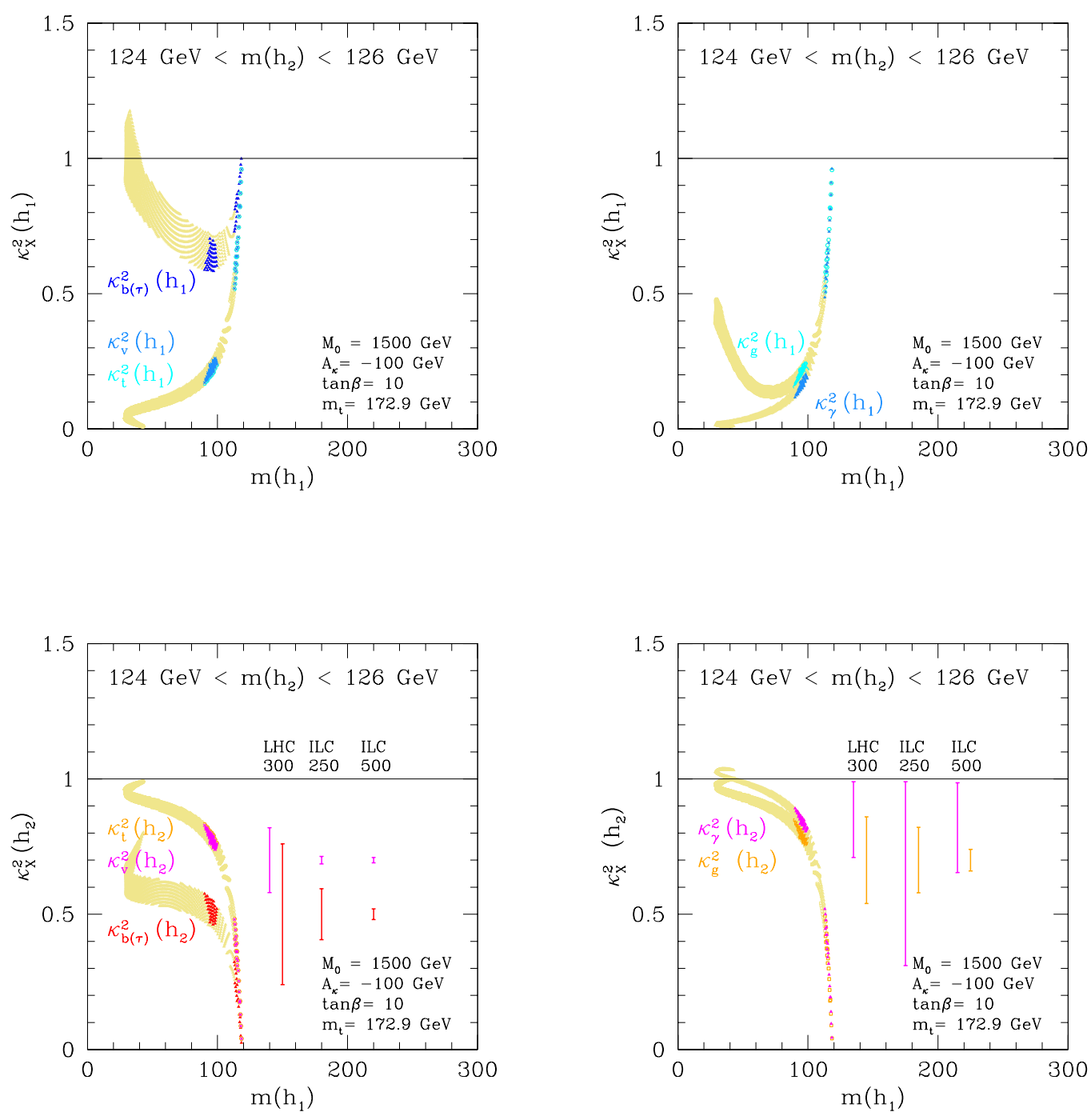

Figure 9. The scale factors of the CP-even Higgs coupling constants for $\tan \beta=10, M_{0}=1500 \mathrm{GeV}$ and $A_{\kappa}=-100 \mathrm{GeV}$. The vertical lines show the expected precisions of the SM-like Higgs coupling constants for LHC and ILC, $\Delta\left(\kappa_{X}\right)^{2} \approx 2 \Delta \kappa_{X} \cdot\left(\kappa_{V}^{1}\right)^{2}$ and $\left(\kappa_{t}^{1}\right)^{2}\left(\left(\kappa_{V}^{2}\right)^{2}\right.$ and $\left.\left(\kappa_{t}^{2}\right)^{2}\right)$ in the upper (lower) left panel almost overlap each other.

In figure 10, we show the scale factors of the lightest CP-odd Higgs boson for the same parameter set with the CP-even bosons. The horizontal axis is chosen as the mass of $a_{1}$, which is rather sensitive to the choice of the PQ symmetry breaking parameter, $A_{\kappa}$. $\left(\kappa_{t}^{\prime 1}\right)^{2}$ and $\left(\kappa_{g}^{\prime 1}\right)^{2}$ are doubly suppressed by $\tan ^{-2} \beta$ and $\sin ^{2} \gamma$ and negligible. The direct production of $a_{1}$ at the LHC is almost hopeless and the gamma-gamma option of ILC may help if $\left(\kappa_{\gamma}^{\prime 1}\right)^{2}$ is large enough.

\subsection{Dark matter}

The existence of the dark matter (DM) is one of the few compelling evidences of physics beyond the SM. In SUSY, the Lightest Supersymmetric Particle (LSP) is stable and good 

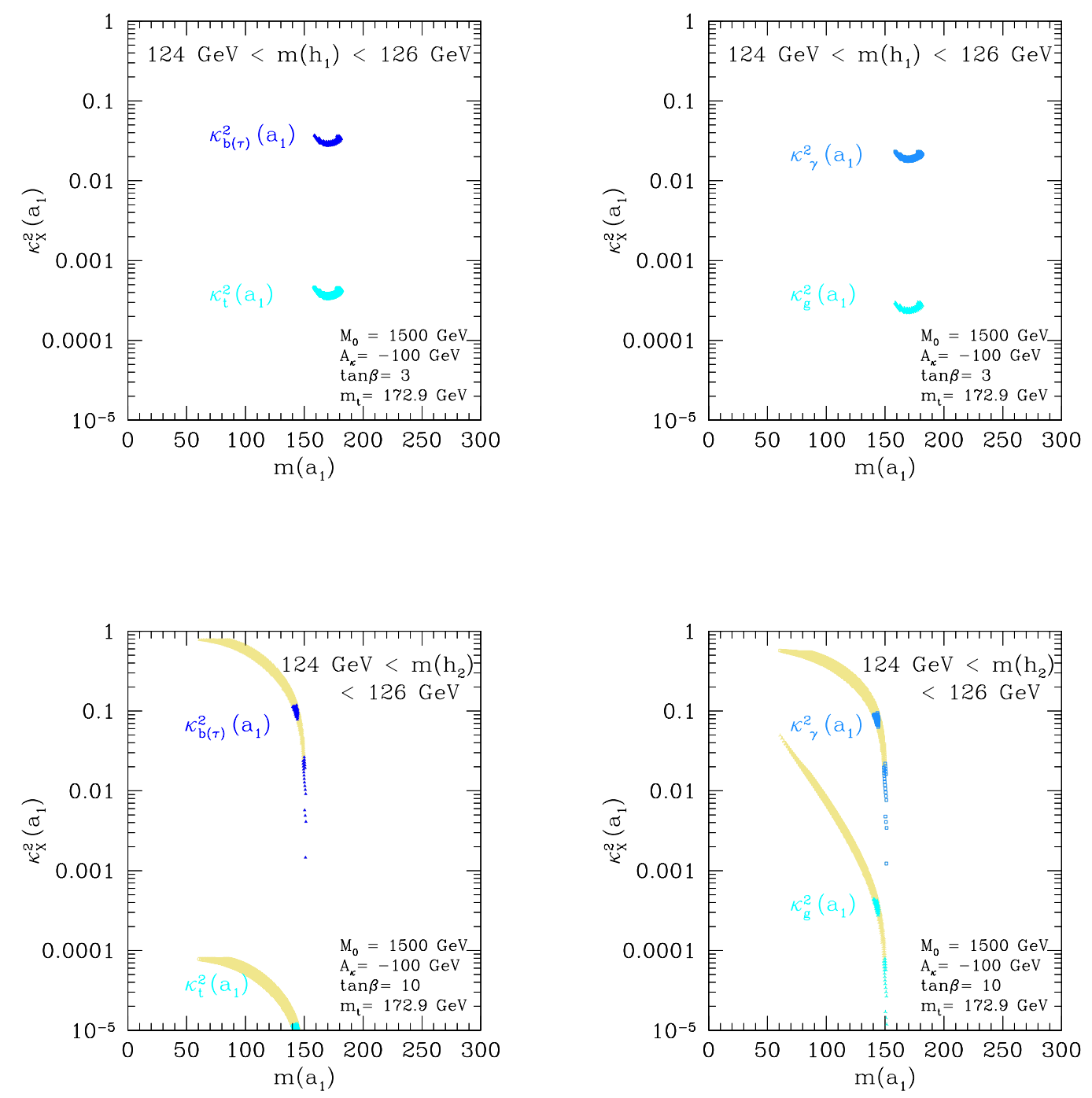

Figure 10. The scale factors for the CP-odd Higgs boson coupling constants for $\tan \beta=3$ (upper panel), $\tan \beta=10$ (lower panel), $M_{0}=1500 \mathrm{GeV}$ and $A_{\kappa}=-100 \mathrm{GeV}$.

candidate of the dark matter, once we assume the R-parity to forbid the dimension 4 baryon/lepton number violating operators to prevent rapid nucleon decay. If LSP is weakly interacting massive particle (WIMP), its annihilation cross section predicts the thermal relic abundance in the ballpark of the observed DM density. This "WIMP miracle" scenario provides a valuable constraint on the SUSY models in light of the recent progress of precision cosmology (for more details, see e.g. [186]). However, in the mirage mediation, the oscillation of the modulus field after the inflation once dominates the energy density of the universe. The reheating temperature of the modulus field is $\mathcal{O}(100) \mathrm{MeV}$. It is much larger than the temperature at which the Big Bang Nucleosynthesis (BBN) takes place, however, lower than the decoupling temperature of LSP. Huge entropy produced by the modulus decay dilutes the preexistent thermal LSP abundance to negligible level. The 
modulus decay produces non-thermal LSP. In particular, daughter gravitino later decays at the decay temperature $\mathcal{O}(10) \mathrm{MeV}$ and overproduces LSP (moduli-induced gravitino problem $[188,189])$. This non-thermal overproduction of DM does not mean the model is excluded, if we consider a specific non-minimal cosmological scenario or modify the DM sector of the model. For example,

1. Suppressing the modulus oscillation or reducing the gravitino branching ratio (e.g. enhanced symmetry [190], adiabatic oscillation [191, 192], alignment [193]).

2. Violating R-parity and introducing another source of DM (e.g. axion [194]) or introducing thermally decoupled light LSP (e.g. axino [86]).

3. Diluting the LSP after the gravitino decay (e.g. by thermal inflation [195-197], Qball [198] or unstable domain wall [199]).

Explicit model building is beyond the scope of this paper. In this section, we just present the prediction of our model with the minimal DM sector assuming the first or the third scenario. The calculation is performed based on micrOMEGAs built in NMSSMTools [143-145].

In our model, LSP is given by mixture of the higgsino and singlino. Generally, the thermal relic of the pure higgsino of $\mathcal{O}(100) \mathrm{GeV}$ cannot saturate the observed DM density. However, mixing with the singlino reduces the annihilation cross section and enables to explain the observed amount of DM (Well-tempered neutralino) in the first scenario as in the case of bino and higgsino in MSSM $[103,200]$. (There is no constraint in the third scenario.)

The searches of DM are classified into the direct and indirect detections. The former detects the scattering of target nucleus by DM at underground laboratories. The latter observes the annihilation/decay products of DM in celestial/astrophysical circumstances such as the center of the earth/sun or galaxies.

We first discuss the prediction for the direct detection. In the direct detection experiments, the scattering between (slow) neutralino and target nucleus proceeds through the scalar or pseudo-vector interactions due to its Majorana nature. The former gives the spin-independent cross section and the latter gives the spin-dependent cross section [186]. In our model, the scalar interaction mainly comes from the exchange of the CP-even Higgs bosons and the pseudo-vector interaction mainly comes from the exchange of the $Z$ boson. The spin-independent nuclear cross section is enhanced by the square of the mass number because the process is coherent, while the spin-dependent cross section does not have such a factor. Thus the experiments are much sensitive to the spin-independent cross section than the spin-dependent ones at nucleon level. On the other hand, the $Z$ boson interacts with nucleon much stronger than the Higgs bosons. Therefore both cross sections could give meaningful constraints on our model, depending on the amount of higgsino component in LSP.

In the upper left panel of figure 11, we show the spin-independent cross section of LSP for $\tan \beta=3$ case. The red region indicates the region where the lightest CP-even boson corresponds to the SM Higgs boson and the blue region indicates the region where the second lightest CP-even boson is the SM-like Higgs boson (although excluded by the other phenomenological constraints). In the pink region, the thermal abundance saturates the observed value of $\mathrm{DM}$ density. Around $m_{L S P} \approx 100,350 \mathrm{GeV}$, the scattering amplitudes 

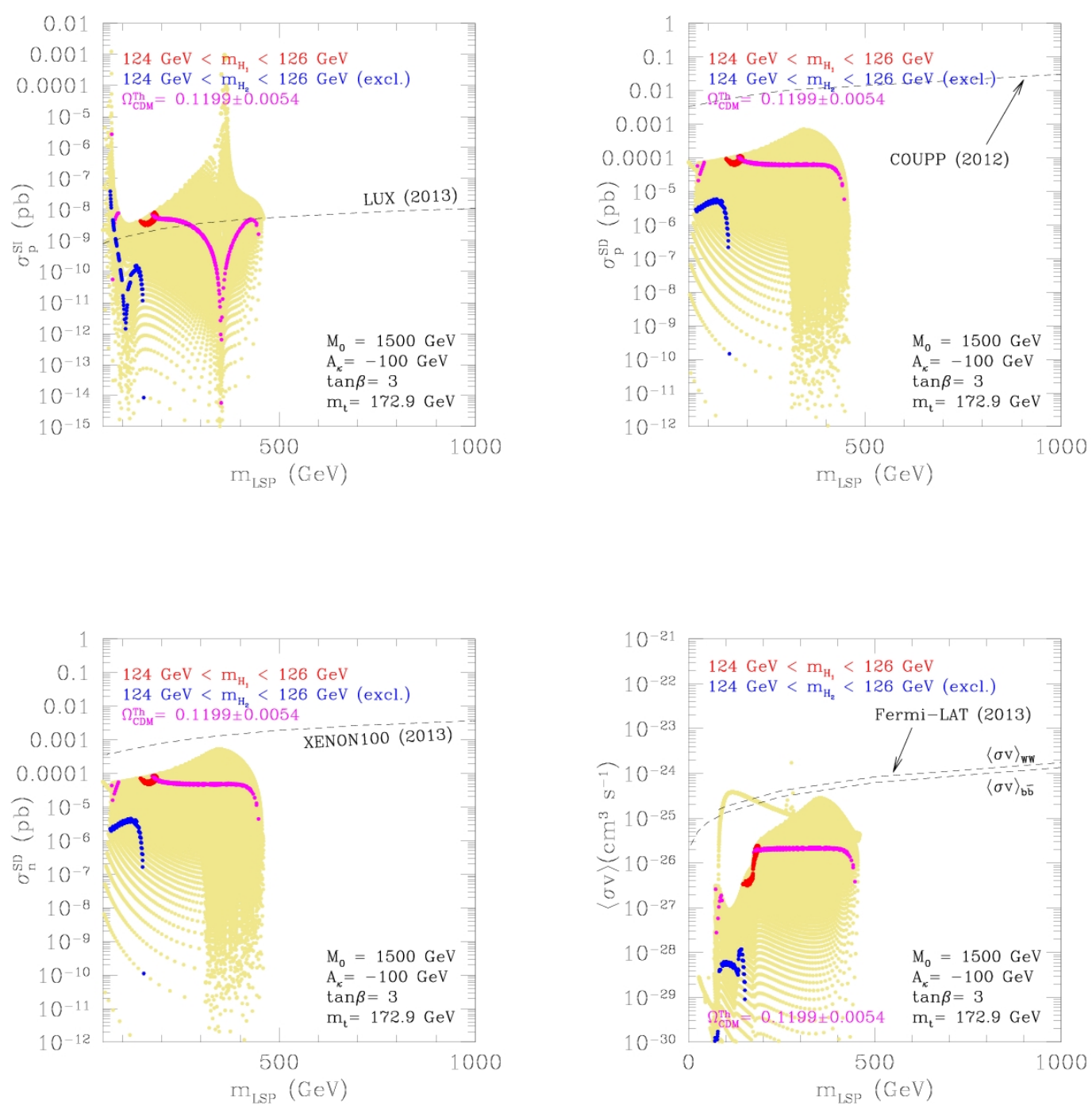

Figure 11. The predictions for the direct and indirect searches of the dark matter for $\tan \beta=3$, $M_{0}=1500 \mathrm{GeV}$ and $A_{\kappa}=-100 \mathrm{GeV}$.

mediated by the lightest and second lightest CP-even bosons cancel each other and the cross section vanishes $[100,201]$. The neutralino-Higgs boson coupling involves $\lambda$, therefore the red region is on the upper edge of the distribution because almost maximum $\lambda$ is required to raise the Higgs mass to $125 \mathrm{GeV}$. With the particular model parameters chosen in the figure, the red region overlaps with the pink region where the thermal abundance saturates the DM, however, is almost excluded by the LUX experiment [202]. If we increases $M_{0}$, the cross section decreases while the pink region remains in the almost same position. In the upper right and lower left panels, we present the spin-dependent cross sections for proton and neutron, respectively. The red region is again on the edge of the distribution because the singlino-higgsino mixing terms in the mass matrix are proportional to $\lambda$. The current experimental bounds are 1-2 order above the model prediction [203, 204]. 

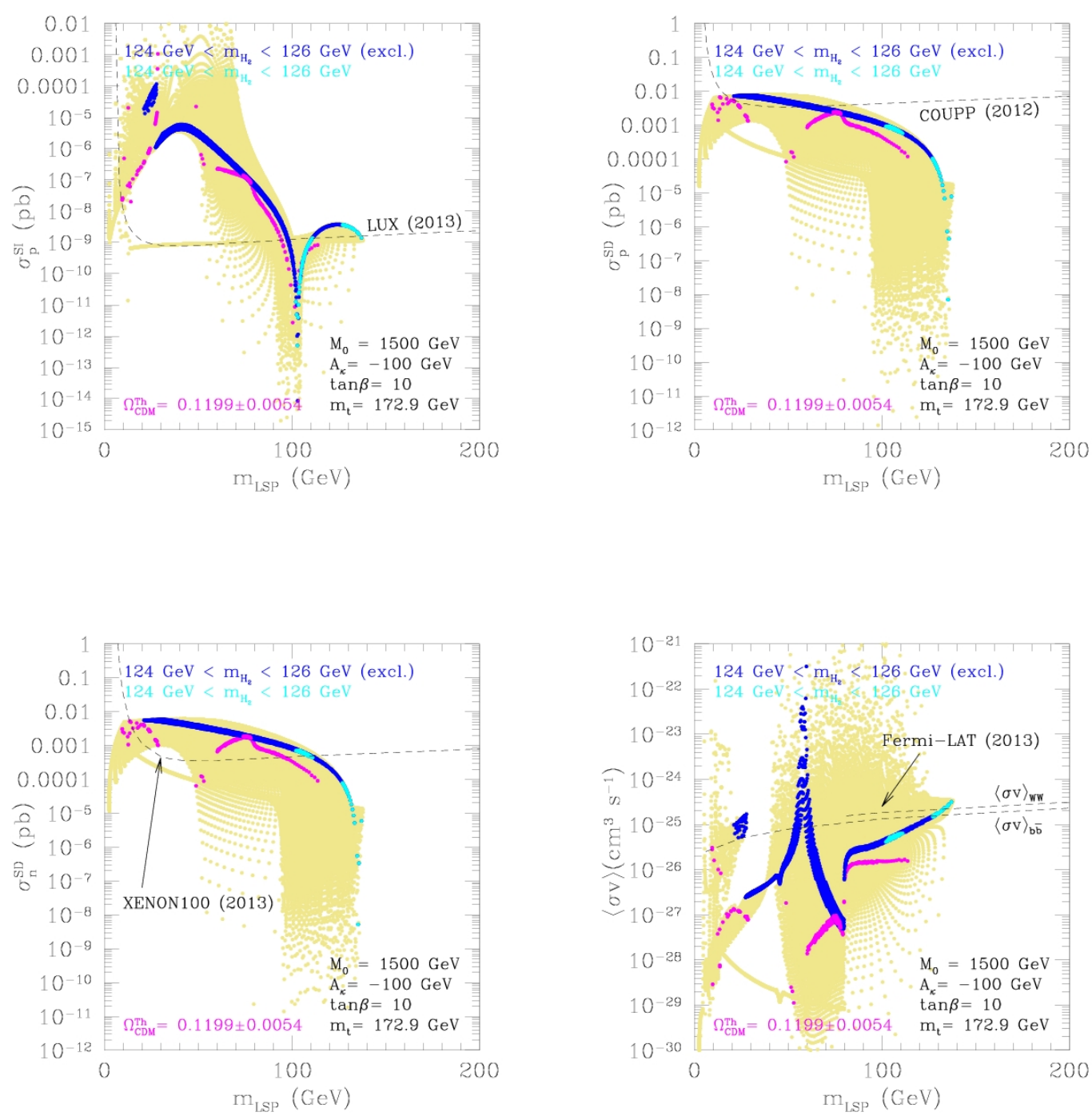

Figure 12. The predictions for the direct and indirect searches of the dark matter for $\tan \beta=10$, $M_{0}=1500 \mathrm{GeV}$ and $A_{\kappa}=-100 \mathrm{GeV}$.

Next we discuss the indirect detection. We consider the constraint by the gamma ray from the dwarf spheroidal galaxies observed by Fermi satellite [205]. The annihilation of the neutralinos mainly produces $W^{+} W^{-}, Z^{0} Z^{0}$ pairs, $t \bar{t}, b \bar{b}$ pairs, $h_{i} a_{j}$ and $h_{i} Z_{j}^{0}$ pairs if they are kinematically available. Other channels are strongly suppressed due to the Majorana nature of the neutralino and CP conservation [186]. In the lower right panel of figure 11, we show the total annihilation cross section of the neutralino and the experimental bound assuming the decay proceeds through $W^{+} W^{-}$and $b \bar{b}$ pairs. In our model, $t \bar{t}$ decay dominates the process above the threshold. The bound is one order larger than the prediction assuming that the gamma ray spectrum from $t \bar{t}$ is not much different from those of $W W, b \bar{b}$ pairs.

Here we impose $124 \mathrm{GeV}<m_{h_{1}}<126 \mathrm{GeV}$, however, the conservative Higgs mass bound, $120 \mathrm{GeV}<m_{h_{1}}<130 \mathrm{GeV}$ does not change the results for $\tan \beta=3$ significantly. 
In figure 12 , we present the same set of the predictions for $\tan \beta=10$ case. The blue and cyan regions indicate the region where the second lightest CP-even boson corresponds to the SM Higgs boson. The blue region is already excluded by the phenomenological constraints explained in the previous section. In the pink region, the thermal LSP abundance saturates the observed DM density. In the surviving cyan region, the annihilation cross section is larger than the value saturating the observed DM density. The spin-independent cross section (upper left panel) shows a dip around $m_{L S P} \approx 100 \mathrm{GeV}$ due to the cancellation between the amplitudes mediated by the two CP even bosons. While the spin-dependent cross sections (upper right and lower left panels) do not show such a behavior because only one diagram dominates the process. They are enhanced with $\tan \beta$ because $\mu \approx M_{0} / \tan \beta$ and the higgsino component in LSP increases with $\tan \beta$. The cyan region is marginally excluded by the spin-independent cross section and the spin-dependent cross section for neutron. Discounting the constraints considering the astrophysical, hadronic and nuclear physics ambiguities in the calculation (see e.g. recent analysis in [187]), it is interesting that the surviving region is exactly located around the dip structure where the fine-tuning of the EW symmetry breaking is also minimized. Once observed, this feature can be checked by the experiment using the target nucleus insensitive to the spin-dependent cross section (enriched even-even nucleus) or combining the results of different target nuclei. In the lower right panel, the resonance structure in the annihilation cross section appears due to the pseudo-scalar mediated $b \bar{b}$ decay, however, this region is excluded by the phenomenological constraints. Above the $W^{+} W^{-}, Z^{0} Z^{0}$ threshold, the process is dominated by $W^{+} W^{-}$and $Z^{0} Z^{0}$ decays. The surviving region is about a factor 2 below the current bound. Once we raise the SUSY scale, $M_{0}$, the constraint from the LEP Higgs boson search becomes weaker until the radiative correction overshoots $125 \mathrm{GeV}$ and the cyan regions in the figures expand, while the EW fine-tuning deteriorates as shown in figure 7. The conservative Higgs mass bound, $120 \mathrm{GeV}<m_{h_{1}}<130 \mathrm{GeV}$ opens a lighter LSP region $\left(m_{L S P} \simeq 50 \mathrm{GeV}\right)$. However, this region is excluded by the upper bound of the spin-independent cross section, if the dominant component of the DM consists of the lightest neutralino.

In this section, we discussed the DM prediction of the model assuming the LSP saturates the observed DM abundance. We stress that the novel structure of the EW symmetry breaking in our model is not directly connected to the DM physics and the model will not be excluded even if the prediction of the minimal model is not confirmed by the experiments.

\section{Conclusion}

We have studied the NMSSM with the TeV scale mirage mediation. Our choice of the modular weights (4.1) realizes a little hierarchy $m_{H_{u}}^{2} \simeq M_{0}^{2} / 8 \pi^{2} \ll M_{0}^{2}$ at the EW scale and automatic cancellation between $\mu^{2}$ and $m_{H_{d}}^{2} / \tan ^{2} \beta$ in (1.1). This leads to a natural EW symmetry breaking with $\mathcal{O}(10) \%$ tuning for the TeV scale stop mass. The $\mu$ term can be as large as $\sqrt{m_{Z} M_{0}}$ without introducing a fine-tuning despite conventional wisdom tells that it lies around the EW scale. The Higgs boson mass is lifted to $125 \mathrm{GeV}$ by the new quartic coupling (small $\tan \beta$ ) or small mixing with the singlet (moderate $\tan \beta$ ) in addition to the radiative corrections (from the top and singlino sectors). The suppression of the 
singlet-doublet mixing is important for raising the Higgs boson mass in the first case and hiding the singlet from the LEP Higgs boson search in the second case. In the NMSSM, if $\kappa=0$ and $m_{S}^{2}=0$, the doublet Higgs VEVs spontaneously break an approximate scale symmetry (4.12). The resultant pseudo Nambu-Goldstone boson (mass eigenstate) almost consists of the doublets and corresponds to the SM-like Higgs with a suppressed singlet component. There is another scale symmetry if $\kappa=0$ and $m_{H_{u}}^{2}=0$ in which $S$ and $H_{u}$ are interchanged. The corresponding Nambu-Goldstone boson is the singlet-like Higgs boson with suppressed $H_{u}$ component. The mixing with the suppressed components must break the two symmetries and disappear if $H_{d}$ decouples. Thus it is highly suppressed although the symmetries are broken at the EW scale. To evaluate the fine-tuning, we introduced a simple formula for the fine-tuning measure having a clear physical meaning which calls for careful treatment of the radiative corrections. Using it, we performed a comprehensive analysis of the fine-tuning in the EW symmetry breaking which shows the model is tuned at most $\mathcal{O}(10) \%$ for $M_{0}=1.5 \mathrm{TeV}$. For moderate $\tan \beta$, the least tuned region is realized with the singlet Higgs boson mass around $100 \mathrm{GeV}$ which overlaps with the anomaly found in the LEP Higgs boson search.

We further investigated the coupling constants of the Higgs bosons in our model. The radiative corrections from the SUSY particles and heavy Higgs bosons are highly suppressed due to the little hierarchy. Thus the deviation from the SM occurs through the suppressed mixing between the SM-like and singlet Higgs bosons. The numerical calculation showed that $20 \%$ deviations are possible in the squares of the scale factors, $\left(\kappa_{X}^{i}\right)^{2}$ and the sum rule $\left(\kappa_{X}^{1}\right)^{2}+\left(\kappa_{X}^{2}\right)^{2}=1$ holds well except for those of $b$ and $\tau$. The mixing with the heavy doublet is also important for $b$ and $\tau$ due to $\tan \beta$ enhancement and an $\mathcal{O}(1)$ deviation is possible for their couplings to the SM-like Higgs boson. Future precision measurements at the LHC and the ILC will provide good opportunities to explore these deviations and the hidden singlet-like scalar.

We also studied prospects for the direct/indirect dark matter searches assuming the minimal dark matter sector in our model. The spin-independent neutralino-nucleus scattering is mainly mediated by the SM-like and singlet-like Higgs bosons. The two amplitudes interfere destructively in some parameter regions. This suppresses the cross section below the current experimental bound. While the spin-dependent scattering is dominated by the $Z$ boson exchange and does not show such a feature. The surviving regions from various phenomenological constraints are almost excluded for $\tan \beta=3$ and on the verge of exclusion for $\tan \beta=10$. The suppression of the spin-independent cross section in the surviving region is characteristic signature to identify the model. The bound on the neutralino annihilation cross section from the gamma ray emission by the dwarf spheroidal galaxy is an order above the prediction and will soon start to explore our model.

\section{Acknowledgments}

We would like to thank Kiwoon Choi very much for helpful comments and discussions. T. K. is supported in part by a Grant-in-Aid for Scientific Research No. 25400252 from the Ministry of Education, Culture, Sports, Science and Technology of Japan. K. O. is 
supported in part by a Grant-in-Aid for Scientific Research No. 21740155 and No. 18071001 from the MEXT of Japan. T. S. is partially supported by a Grant-in-Aid for Young Scientists (B) No. 23740190 and a Grant-in-Aid for Scientific Research No. 15K17645 from the MEXT Japan. Numerical computation in this work was partly carried out at the Yukawa Institute Computer Facility.

\section{A Soft SUSY breaking terms}

Here we give explicitly soft SUSY breaking terms induced by the mirage mediation mechanism in the NMSSM.

In the mirage mediation, the soft parameters at the scale just below $M_{\mathrm{GUT}}$ are given by

$$
\begin{aligned}
M_{a}\left(M_{\mathrm{GUT}}\right) & =M_{0}+\frac{m_{3 / 2}}{8 \pi^{2}} b_{a} g_{a}^{2}, \\
A_{i j k}\left(M_{\mathrm{GUT}}\right) & =\left(c_{i}+c_{j}+c_{k}\right) M_{0}-\left(\gamma_{i}+\gamma_{j}+\gamma_{k}\right) \frac{m_{3 / 2}}{8 \pi^{2}}, \\
m_{i}^{2}\left(M_{\mathrm{GUT}}\right) & =c_{i} M_{0}^{2}-\dot{\gamma}_{i}\left(\frac{m_{3 / 2}}{8 \pi^{2}}\right)^{2}-\frac{m_{3 / 2}}{8 \pi^{2}} M_{0} \theta_{i},
\end{aligned}
$$

where

$$
\begin{aligned}
b_{a} & =-3 \operatorname{tr}\left(T_{a}^{2}(\operatorname{Adj})\right)+\sum_{i} \operatorname{tr}\left(T_{a}^{2}\left(\phi^{i}\right)\right), \\
\gamma_{i} & =2 \sum_{a} g_{a}^{2} C_{2}^{a}\left(\phi^{i}\right)-\frac{1}{2} \sum_{j k}\left|y_{i j k}\right|^{2}, \\
\theta_{i} & =4 \sum_{a} g_{a}^{2} C_{2}^{a}\left(\phi^{i}\right)-\sum_{j k} a_{i j k}\left|y_{i j k}\right|^{2}, \\
\dot{\gamma}_{i} & =8 \pi^{2} \frac{d \gamma_{i}}{d \ln \mu_{R}} .
\end{aligned}
$$

Here, $T_{a}^{2}(\mathrm{Adj})$ and $T_{a}^{2}\left(\phi^{i}\right)$ denote Dynkin indices of the adjoint representation and the representation of matter fields $\phi^{i}$. We have assumed $\omega_{i j}=\Sigma_{k l} y_{i j k} y_{j k l}^{*}$ to be diagonal.

Within the framework of the NMSSM, the $\beta$-function coefficients, anomalous dimensions and other coefficients in the above equations are obtained as

$$
\begin{aligned}
b_{3} & =-3, b_{2}=1, b_{1}=11, \\
\gamma_{H_{u}} & =\frac{3}{2} g_{2}^{2}+\frac{1}{2} g_{1}^{2}-3 y_{t}^{2}-\lambda^{2}, \\
\gamma_{H_{d}} & =\frac{3}{2} g_{2}^{2}+\frac{1}{2} g_{1}^{2}-\lambda^{2}, \\
\gamma_{S} & =-2 \kappa^{2}-2 \lambda^{2}, \\
\gamma_{Q_{a}} & =\frac{8}{3} g_{3}^{2}+\frac{3}{2} g_{2}^{2}+\frac{1}{18} g_{1}^{2}-\left(y_{t}^{2}+y_{b}^{2}\right) \delta_{3 a}, \\
\gamma_{U_{a}} & =\frac{8}{3} g_{3}^{2}+\frac{8}{9} g_{1}^{2}-2 y_{t}^{2} \delta_{3 a}, \\
\gamma_{D_{a}} & =\frac{8}{3} g_{3}^{2}+\frac{2}{9} g_{1}^{2}-2 y_{b}^{2} \delta_{3 a},
\end{aligned}
$$




$$
\begin{aligned}
\gamma_{L_{a}} & =\frac{3}{2} g_{2}^{2}+\frac{1}{2} g_{1}^{2}-y_{\tau}^{2} \delta_{3 a} \\
\gamma_{E_{a}} & =2 g_{1}^{2}-2 y_{\tau}^{2} \delta_{3 a}
\end{aligned}
$$

$$
\begin{aligned}
\theta_{H_{u}} & =3 g_{2}^{2}+g_{1}^{2}-6 y_{t}^{2} a_{H_{u} Q_{3} U_{3}^{c}}-2 \lambda^{2} a_{H_{u} H_{d} S} \\
\theta_{H_{d}} & =3 g_{2}^{2}+g_{1}^{2}-6 y_{b}^{2} a_{H_{d} Q_{3} D_{3}^{c}}-2 y_{\tau}^{2} a_{H_{d} L_{3} E_{3}^{c}}-2 \lambda^{2} a_{H_{u} H_{d} S}, \\
\theta_{S} & =-2 \lambda^{2} a_{H_{u} H_{d} S}-\kappa^{2} a_{S S S} \\
\theta_{Q_{a}} & =\frac{16}{3} g_{3}^{2}+3 g_{2}^{2}+\frac{1}{9} g_{1}^{2}-2\left(y_{t}^{2} a_{H_{u} Q_{3} U_{3}^{c}}+y_{b}^{2} a_{\left.H_{d} Q_{3} D_{3}^{c}\right) \delta_{3 a}}\right. \\
\theta_{U_{a}} & =\frac{16}{3} g_{3}^{2}+\frac{16}{9} g_{1}^{2}-4 y_{t}^{2} a_{H_{u} Q_{3} U_{3}^{c} \delta_{3 a}} \\
\theta_{D_{a}} & =\frac{16}{3} g_{3}^{2}+\frac{4}{9} g_{1}^{2}-4 y_{b}^{2} a_{H_{d} Q_{3} D_{3}^{c} \delta_{3 a}} \\
\theta_{L_{a}} & =3 g_{2}^{2}+g_{1}^{2}-2 y_{\tau}^{2} a_{H_{d} L_{3} E_{3}^{c}} \delta_{3 a} \\
\theta_{E_{a}} & =4 g_{1}^{2}-4 y_{\tau}^{2} a_{H_{d} L_{3} E_{3}^{c} \delta_{3 a}}
\end{aligned}
$$

$$
\begin{aligned}
\dot{\gamma}_{H_{u}} & =\frac{3}{2} g_{2}^{4}+\frac{11}{2} g_{1}^{4}-3 y_{t}^{2} b_{y_{t}}-\lambda^{2} b_{\lambda}, \\
\dot{\gamma}_{H_{d}} & =\frac{3}{2} g_{2}^{4}+\frac{11}{2} g_{1}^{4}-3 y_{b}^{2} b_{y_{b}}-y_{\tau}^{2} b_{y_{\tau}}-\lambda^{2} b_{\lambda}, \\
\dot{\gamma}_{S} & =-2 \kappa^{2} b_{\kappa}-2 \lambda^{2} b_{\lambda}, \\
\dot{\gamma}_{Q_{a}} & =-8 g_{3}^{4}+\frac{3}{2} g_{2}^{4}+\frac{11}{18} g_{1}^{4}-\left(y_{t}^{2} b_{y_{t}}+y_{b}^{2} b_{y_{b}}\right) \delta_{3 a}, \\
\dot{\gamma}_{U_{a}} & =-8 g_{3}^{4}+\frac{88}{9} g_{1}^{4}-2 y_{t}^{2} b_{y_{t}} \delta_{3 a}, \\
\dot{\gamma}_{D_{a}} & =-8 g_{3}^{4}+\frac{22}{9} g_{1}^{4}-2 y_{b}^{2} b_{y_{b}} \delta_{3 a}, \\
\dot{\gamma}_{L_{a}} & =\frac{3}{2} g_{2}^{4}+\frac{11}{2} g_{1}^{4}-y_{\tau}^{2} b_{y_{\tau}} \delta_{3 a}, \\
\dot{\gamma}_{E_{a}} & =22 g_{1}^{4}-2 y_{\tau}^{2} b_{y_{\tau}} \delta_{3 a},
\end{aligned}
$$

where

$$
\begin{aligned}
b_{y_{t}} & =-\frac{16}{3} g_{3}^{2}-3 g_{2}^{2}-\frac{13}{9} g_{1}^{2}+6 y_{t}^{2}+y_{b}^{2}+\lambda^{2}, \\
b_{y_{b}} & =-\frac{16}{3} g_{3}^{2}-3 g_{2}^{2}-\frac{7}{9} g_{1}^{2}+y_{t}^{2}+6 y_{b}^{2}+y_{\tau}^{2}+\lambda^{2}, \\
b_{y_{\tau}} & =-3 g_{2}^{2}-3 g_{1}^{2}+3 y_{b}^{2}+4 y_{\tau}^{2}+\lambda^{2} \\
b_{\kappa} & =6 \lambda^{2}+6 \kappa^{2} \\
b_{\lambda} & =-3 g_{2}^{2}-g_{1}^{2}+3 y_{t}^{2}+3 y_{b}^{2}+y_{\tau}^{2}+4 \lambda^{2}+2 \kappa^{2} .
\end{aligned}
$$

Here, $Q_{a}, U_{a}, D_{a}, L_{a}$, and $E_{a}$ denote left-handed quark, right-handed up-sector quark, righthanded down-sector quark, left-handed lepton, and right-handed lepton fields, respectively, and the index $a$ denotes the generation index. We have included effects due to Yukawa couplings, $y_{t}, y_{b}$, and $y_{\tau}$, only for the third generations. 
Open Access. This article is distributed under the terms of the Creative Commons Attribution License (CC-BY 4.0), which permits any use, distribution and reproduction in any medium, provided the original author(s) and source are credited.

\section{References}

[1] ATLAS collaboration, Observation of a new particle in the search for the standard model Higgs boson with the ATLAS detector at the LHC, Phys. Lett. B 716 (2012) 1 [arXiv:1207.7214] [INSPIRE].

[2] CMS collaboration, Observation of a new boson at a mass of $125 \mathrm{GeV}$ with the CMS experiment at the LHC, Phys. Lett. B 716 (2012) 30 [arXiv:1207.7235] [INSPIRE].

[3] ATLAS and CMS collaborations, Combined measurement of the Higgs boson mass in pp collisions at $\sqrt{s}=7$ and $8 \mathrm{TeV}$ with the ATLAS and CMS experiments, Phys. Rev. Lett. 114 (2015) 191803 [arXiv: 1503.07589] [INSPIRE].

[4] CMS collaboration, Study of the mass and spin-parity of the Higgs boson candidate via its decays to $Z$ boson pairs, Phys. Rev. Lett. 110 (2013) 081803 [arXiv:1212.6639] [InSPIRE].

[5] ATLAS collaboration, Evidence for the spin-0 nature of the Higgs boson using ATLAS data, Phys. Lett. B 726 (2013) 120 [arXiv:1307.1432] [INSPIRE].

[6] CMS collaboration, Constraints on the spin-parity and anomalous HVV couplings of the Higgs boson in proton collisions at 7 and 8 TeV, Phys. Rev. D 92 (2015) 012004 [arXiv: 1411.3441] [INSPIRE].

[7] ATLAS collaboration, Determination of spin and parity of the Higgs boson in the $W W^{*} \rightarrow e \nu \mu \nu$ decay channel with the ATLAS detector, Eur. Phys. J. C 75 (2015) 231 [arXiv: 1503.03643] [INSPIRE].

[8] ATLAS collaboration, Measurements of the Higgs boson production and decay rates and coupling strengths using pp collision data at $\sqrt{s}=7$ and $8 \mathrm{TeV}$ in the ATLAS experiment, ATLAS-CONF-2015-007, CERN, Geneva Switzerland (2015).

[9] CMS collaboration, Precise determination of the mass of the Higgs boson and tests of compatibility of its couplings with the standard model predictions using proton collisions at 7 and 8 TeV, Eur. Phys. J. C 75 (2015) 212 [arXiv:1412.8662] [inSPIRE].

[10] ATLAS collaboration, Search for squarks and gluinos with the ATLAS detector in final states with jets and missing transverse momentum using $\sqrt{s}=8$ TeV proton-proton collision data, JHEP 09 (2014) 176 [arXiv:1405.7875] [INSPIRE].

[11] ATLAS collaboration, Search for squarks and gluinos in events with isolated leptons, jets and missing transverse momentum at $\sqrt{s}=8 \mathrm{TeV}$ with the ATLAS detector, JHEP 04 (2015) 116 [arXiv:1501.03555] [INSPIRE].

[12] ATLAS collaboration, Search for supersymmetry in events containing a same-flavour opposite-sign dilepton pair, jets and large missing transverse momentum in $\sqrt{s}=8 \mathrm{TeV} p p$ collisions with the ATLAS detector, Eur. Phys. J. C 75 (2015) 318 [arXiv:1503.03290] [INSPIRE].

[13] ATLAS collaboration, Search for supersymmetry in events with large missing transverse momentum, jets and at least one $\tau$ lepton in $20 \mathrm{fb}^{-1}$ of $\sqrt{s}=8 \mathrm{TeV}$ proton-proton collision data with the ATLAS detector, JHEP 09 (2014) 103 [arXiv:1407.0603] [INSPIRE]. 
[14] CMS collaboration, Search for supersymmetry in pp collisions at $\sqrt{s}=8 \mathrm{TeV}$ in events with a single lepton, large jet multiplicity and multiple b jets, Phys. Lett. B 733 (2014) 328 [arXiv:1311.4937] [INSPIRE].

[15] CMS collaboration, Search for new physics in events with same-sign dileptons and jets in pp collisions at $\sqrt{s}=8 \mathrm{TeV}$, JHEP 01 (2014) 163 [Erratum ibid. 01 (2015) 014] [arXiv: 1311.6736] [INSPIRE].

[16] ATLAS collaboration, Search for new phenomena in final states with large jet multiplicities and missing transverse momentum at $\sqrt{s}=8 \mathrm{TeV}$ proton-proton collisions using the ATLAS experiment, JHEP 10 (2013) 130 [Erratum ibid. 01 (2014) 109] [arXiv:1308.1841] [INSPIRE].

[17] ATLAS collaboration, Search for strong production of supersymmetric particles in final states with missing transverse momentum and at least three b-jets at $\sqrt{s}=8 \mathrm{TeV}$ proton-proton collisions with the ATLAS detector, JHEP 10 (2014) 024 [arXiv:1407.0600] [INSPIRE].

[18] CMS collaboration, Search for supersymmetry in pp collisions at $\sqrt{s}=8 \mathrm{TeV}$ in events with a single lepton, large jet multiplicity and multiple b-jets, Phys. Lett. B 733 (2014) 328 [arXiv:1311.4937] [INSPIRE].

[19] CMS collaboration, Search for supersymmetry in pp collisions at $\sqrt{s}=8 \mathrm{TeV}$ in events with three leptons and at least one b-tagged jet, CMS-PAS-SUS-13-008, CERN, Geneva Switzerland (2013).

[20] CMS collaboration, Exclusion limits on gluino and top-squark pair production in natural SUSY scenarios with inclusive razor and exclusive single-lepton searches at $8 \mathrm{TeV}$, CMS-PAS-SUS-14-011, CERN, Geneva Switzerland (2014).

[21] ATLAS collaboration, Search for top squark pair production in final states with one isolated lepton, jets and missing transverse momentum in $\sqrt{s}=8 \mathrm{TeV}$ pp collisions with the ATLAS detector, JHEP 11 (2014) 118 [arXiv:1407.0583] [INSPIRE].

[22] ATLAS collaboration, Search for direct pair production of the top squark in all-hadronic final states in proton-proton collisions at $\sqrt{s}=8 \mathrm{TeV}$ with the ATLAS detector, JHEP 09 (2014) 015 [arXiv: 1406.1122] [inSPIRE].

[23] ATLAS collaboration, Search for direct top-squark pair production in final states with two leptons in pp collisions at $\sqrt{s}=8 \mathrm{TeV}$ with the ATLAS detector, JHEP 06 (2014) 124 [arXiv: 1403.4853] [INSPIRE].

[24] ATLAS collaboration, Search for direct third-generation squark pair production in final states with missing transverse momentum and two b-jets in $\sqrt{s}=8$ TeV pp collisions with the ATLAS detector, JHEP 10 (2013) 189 [arXiv:1308.2631] [INSPIRE].

[25] ATLAS collaboration, Search for light scalar top quark pair production in final states with two leptons with the ATLAS detector in $\sqrt{s}=7 \mathrm{TeV}$ proton-proton collisions, Eur. Phys. J. C 72 (2012) 2237 [arXiv:1208.4305] [INSPIRE].

[26] ATLAS collaboration, Search for light top squark pair production in final states with leptons and b-jets with the ATLAS detector in $\sqrt{s}=7 \mathrm{TeV}$ proton-proton collisions, Phys. Lett. B 720 (2013) 13 [arXiv:1209.2102] [INSPIRE].

[27] ATLAS collaboration, Search for pair-produced third-generation squarks decaying via charm quarks or in compressed supersymmetric scenarios in pp collisions at $\sqrt{s}=8 \mathrm{TeV}$ with the ATLAS detector, Phys. Rev. D 90 (2014) 052008 [arXiv:1407.0608] [InSPIRE]. 
[28] ATLAS collaboration, Search for direct top squark pair production in events with a $Z$ boson, $b$-jets and missing transverse momentum in $\sqrt{s}=8 \mathrm{TeV}$ pp collisions with the ATLAS detector, Eur. Phys. J. C 74 (2014) 2883 [arXiv:1403.5222] [InSPIRE].

[29] CMS collaboration, Search for top-squark pair production in the single-lepton final state in pp collisions at $\sqrt{s}=8 \mathrm{TeV}$, Eur. Phys. J. C 73 (2013) 2677 [arXiv:1308.1586] [InSPIRE].

[30] CMS collaboration, Search for top squark and higgsino production using diphoton Higgs boson decays, Phys. Rev. Lett. 112 (2014) 161802 [arXiv:1312.3310] [INSPIRE].

[31] CMS collaboration, Search for supersymmetry using razor variables in events with b-tagged jets in pp collisions at $\sqrt{s}=8 \mathrm{TeV}$, Phys. Rev. D 91 (2015) 052018 [arXiv:1502.00300] [INSPIRE].

[32] CMS collaboration, Search for top-squark pairs decaying into Higgs or $Z$ bosons in pp collisions at $\sqrt{s}=8 \mathrm{TeV}$, Phys. Lett. B 736 (2014) 371 [arXiv:1405.3886] [inSPIRE].

[33] ATLAS collaboration, Search for supersymmetry at $\sqrt{s}=8 \mathrm{TeV}$ in final states with jets and two same-sign leptons or three leptons with the ATLAS detector, JHEP 06 (2014) 035 [arXiv:1404.2500] [INSPIRE].

[34] ATLAS collaboration, Search for direct third-generation squark pair production in final states with missing transverse momentum and two b-jets in $\sqrt{s}=8 \mathrm{TeV}$ pp collisions with the ATLAS detector, JHEP 10 (2013) 189 [arXiv:1308.2631] [INSPIRE].

[35] CMS collaboration, Search for direct production of bottom squark pairs, CMS-PAS-SUS-13-018, CERN, Geneva Switzerland (2013).

[36] CMS collaboration, Search for supersymmetry in pp collisions at $\sqrt{s}=8 \mathrm{TeV}$ in events with three leptons and at least one b-tagged jet, CMS-PAS-SUS-13-008, CERN, Geneva Switzerland (2013).

[37] CMS collaboration, Search for new physics in events with same-sign dileptons, CMS-PAS-SUS-13-013, CERN, Geneva Switzerland (2013).

[38] K. Choi, A. Falkowski, H.P. Nilles, M. Olechowski and S. Pokorski, Stability of flux compactifications and the pattern of supersymmetry breaking, JHEP 11 (2004) 076 [hep-th/0411066] [INSPIRE].

[39] K. Choi, A. Falkowski, H.P. Nilles and M. Olechowski, Soft supersymmetry breaking in KKLT flux compactification, Nucl. Phys. B 718 (2005) 113 [hep-th/0503216] [INSPIRE].

[40] K. Choi, K.S. Jeong and K.-I. Okumura, Phenomenology of mixed modulus-anomaly mediation in fluxed string compactifications and brane models, JHEP 09 (2005) 039 [hep-ph/0504037] [INSPIRE].

[41] M. Endo, M. Yamaguchi and K. Yoshioka, A bottom-up approach to moduli dynamics in heavy gravitino scenario: superpotential, soft terms and sparticle mass spectrum, Phys. Rev. D 72 (2005) 015004 [hep-ph/0504036] [INSPIRE].

[42] A. Falkowski, O. Lebedev and Y. Mambrini, SUSY phenomenology of KKLT flux compactifications, JHEP 11 (2005) 034 [hep-ph/0507110] [INSPIRE].

[43] O. Lebedev, H.P. Nilles and M. Ratz, A note on fine-tuning in mirage mediation, hep-ph/0511320 [INSPIRE].

[44] H. Baer, E.-K. Park, X. Tata and T.T. Wang, Collider and dark matter searches in models with mixed modulus-anomaly mediated SUSY breaking, JHEP 08 (2006) 041

[hep-ph/0604253] [INSPIRE]. 
[45] H. Baer, E.-K. Park, X. Tata and T.T. Wang, Collider and dark matter phenomenology of models with mirage unification, JHEP 06 (2007) 033 [hep-ph/0703024] [INSPIRE].

[46] R. Kitano and Y. Nomura, Supersymmetry, naturalness and signatures at the LHC, Phys. Rev. D 73 (2006) 095004 [hep-ph/0602096] [INSPIRE].

[47] A. Pierce and J. Thaler, Prospects for mirage mediation, JHEP 09 (2006) 017 [hep-ph/0604192] [INSPIRE].

[48] K. Kawagoe and M.M. Nojiri, Discovery of supersymmetry with degenerated mass spectrum, Phys. Rev. D 74 (2006) 115011 [hep-ph/0606104] [INSPIRE].

[49] H. Baer, E.-K. Park, X. Tata and T.T. Wang, Measuring modular weights in mirage unification models at the LHC and ILC, Phys. Lett. B 641 (2006) 447 [hep-ph/0607085] [INSPIRE].

[50] H. Baer, E.-K. Park, X. Tata and T.T. Wang, Collider and dark matter phenomenology of models with mirage unification, JHEP 06 (2007) 033 [hep-ph/0703024] [INSPIRE].

[51] W.S. Cho, Y.G. Kim, K.Y. Lee, C.B. Park and Y. Shimizu, LHC signature of mirage mediation, JHEP 04 (2007) 054 [hep-ph/0703163] [INSPIRE].

[52] H. Abe, Y.G. Kim, T. Kobayashi and Y. Shimizu, TeV scale partial mirage unification and neutralino dark matter, JHEP 09 (2007) 107 [arXiv:0706.4349] [INSPIRE].

[53] V. Lowen and H.P. Nilles, Mirage pattern from the heterotic string, Phys. Rev. D 77 (2008) 106007 [arXiv:0802.1137] [INSPIRE].

[54] L.L. Everett, I.-W. Kim, P. Ouyang and K.M. Zurek, Deflected mirage mediation: a framework for generalized supersymmetry breaking, Phys. Rev. Lett. 101 (2008) 101803 [arXiv: 0804.0592] [INSPIRE].

[55] L.L. Everett, I.-W. Kim, P. Ouyang and K.M. Zurek, Moduli stabilization and supersymmetry breaking in deflected mirage mediation, JHEP 08 (2008) 102 [arXiv: 0806.2330] [INSPIRE].

[56] K. Choi, K.S. Jeong, S. Nakamura, K.-I. Okumura and M. Yamaguchi, Sparticle masses in deflected mirage mediation, JHEP 04 (2009) 107 [arXiv:0901.0052] [INSPIRE].

[57] B. Dutta, L. Leblond and K. Sinha, Mirage in the sky: non-thermal dark matter, gravitino problem and cosmic ray anomalies, Phys. Rev. D 80 (2009) 035014 [arXiv:0904.3773] [INSPIRE].

[58] B. Altunkaynak, B.D. Nelson, L.L. Everett, I.-W. Kim and Y. Rao, Phenomenological implications of deflected mirage mediation: comparison with mirage mediation, JHEP 05 (2010) 054 [arXiv: 1001.5261] [INSPIRE].

[59] B. Altunkaynak, B.D. Nelson, L.L. Everett, Y. Rao and I.-W. Kim, Landscape of supersymmetric particle mass hierarchies in deflected mirage mediation, Eur. Phys. J. Plus 127 (2012) 2 [arXiv:1011.1439] [INSPIRE].

[60] B.L. Kaufman, B.D. Nelson and M.K. Gaillard, Mirage models confront the LHC: Kähler-stabilized heterotic string theory, Phys. Rev. D 88 (2013) 025003 [arXiv:1303.6575] [INSPIRE].

[61] B. Kaufman and B.D. Nelson, Mirage models confront the LHC: II. Flux-stabilized type IIB string theory, Phys. Rev. D 89 (2014) 085029 [arXiv: 1312.6621] [INSPIRE]. 
[62] H. Abe and J. Kawamura, The $126 \mathrm{GeV}$ Higgs boson mass and naturalness in (deflected) mirage mediation, JHEP 07 (2014) 077 [arXiv: 1405.0779] [INSPIRE].

[63] K. Huitu, P.N. Pandita and P. Tiitola, Renormalization group invariants and sum rules in the deflected mirage mediation supersymmetry breaking, Phys. Rev. D 92 (2015) 075037 [arXiv:1505.03455] [INSPIRE].

[64] V.S. Kaplunovsky and J. Louis, Model independent analysis of soft terms in effective supergravity and in string theory, Phys. Lett. B 306 (1993) 269 [hep-th/9303040] [INSPIRE].

[65] A. Brignole, L.E. Ibáñez and C. Muñoz, Towards a theory of soft terms for the supersymmetric standard model, Nucl. Phys. B 422 (1994) 125 [Erratum ibid. B 436 (1995) 747] [hep-ph/9308271] [INSPIRE].

[66] T. Kobayashi, D. Suematsu, K. Yamada and Y. Yamagishi, Nonuniversal soft scalar masses in superstring theories, Phys. Lett. B 348 (1995) 402 [hep-ph/9408322] [INSPIRE].

[67] L.E. Ibáñez, C. Muñoz and S. Rigolin, Aspect of type-I string phenomenology, Nucl. Phys. B 553 (1999) 43 [hep-ph/9812397] [INSPIRE].

[68] L. Randall and R. Sundrum, Out of this world supersymmetry breaking, Nucl. Phys. B 557 (1999) 79 [hep-th/9810155] [INSPIRE].

[69] G.F. Giudice, M.A. Luty, H. Murayama and R. Rattazzi, Gaugino mass without singlets, JHEP 12 (1998) 027 [hep-ph/9810442] [INSPIRE].

[70] K. Choi, K.S. Jeong, T. Kobayashi and K.-I. Okumura, Little SUSY hierarchy in mixed modulus-anomaly mediation, Phys. Lett. B 633 (2006) 355 [hep-ph/0508029] [INSPIRE].

[71] R. Kitano and Y. Nomura, A solution to the supersymmetric fine-tuning problem within the MSSM, Phys. Lett. B 631 (2005) 58 [hep-ph/0509039] [INSPIRE].

[72] K. Choi, K.S. Jeong, T. Kobayashi and K.-I. Okumura, TeV scale mirage mediation and natural little SUSY hierarchy, Phys. Rev. D 75 (2007) 095012 [hep-ph/0612258] [INSPIRE].

[73] K.L. Chan, U. Chattopadhyay and P. Nath, Naturalness, weak scale supersymmetry and the prospect for the observation of supersymmetry at the Tevatron and at the CERN LHC, Phys. Rev. D 58 (1998) 096004 [hep-ph/9710473] [INSPIRE].

[74] J.L. Feng, K.T. Matchev and T. Moroi, Focus points and naturalness in supersymmetry, Phys. Rev. D 61 (2000) 075005 [hep-ph/9909334] [INSPIRE].

[75] G.L. Kane, J.D. Lykken, B.D. Nelson and L.-T. Wang, Reexamination of electroweak symmetry breaking in supersymmetry and implications for light superpartners, Phys. Lett. B 551 (2003) 146 [hep-ph/0207168] [INSPIRE].

[76] H. Abe, T. Kobayashi and Y. Omura, Relaxed fine-tuning in models with non-universal gaugino masses, Phys. Rev. D 76 (2007) 015002 [hep-ph/0703044] [INSPIRE].

[77] S. Antusch, L. Calibbi, V. Maurer, M. Monaco and M. Spinrath, Naturalness of the non-universal MSSM in the light of the recent Higgs results, JHEP 01 (2013) 187 [arXiv:1207.7236] [INSPIRE].

[78] H. Abe, J. Kawamura and H. Otsuka, The Higgs boson mass in a natural MSSM with nonuniversal gaugino masses at the GUT scale, Prog. Theor. Exp. Phys. 2013 (2013) 013B02 [arXiv: 1208.5328] [inSPIRE].

[79] D. Horton and G.G. Ross, Naturalness and focus points with non-universal gaugino masses, Nucl. Phys. B 830 (2010) 221 [arXiv:0908.0857] [INSPIRE]. 
[80] F. Brümmer and W. Buchmüller, A low Fermi scale from a simple gaugino-scalar mass relation, JHEP 03 (2014) 075 [arXiv: 1311.1114] [INSPIRE].

[81] T.T. Yanagida and N. Yokozaki, Focus point in gaugino mediation - reconsideration of the fine-tuning problem, Phys. Lett. B 722 (2013) 355 [arXiv:1301.1137] [INSPIRE].

[82] T.T. Yanagida and N. Yokozaki, Bino-Higgsino mixed dark matter in a focus point gaugino mediation, JHEP 11 (2013) 020 [arXiv:1308.0536] [INSPIRE].

[83] T.T. Yanagida and N. Yokozaki, Upper bounds on gluino, squark and Higgisino masses in the focus point gaugino mediation with a mild fine tuning $\Delta \leq 100$, JHEP 10 (2014) 133 [arXiv: 1404.2025] [INSPIRE].

[84] K. Harigaya, T.T. Yanagida and N. Yokozaki, Muon g-2 in focus point SUSY, Phys. Rev. D 92 (2015) 035011 [arXiv: 1505.01987] [INSPIRE].

[85] J. Louis and L. Zarate, Hiding the little hierarchy problem in the NMSSM, JHEP 08 (2015) 062 [arXiv: 1506.01616] [INSPIRE].

[86] S. Nakamura, K.-I. Okumura and M. Yamaguchi, Axionic mirage mediation, Phys. Rev. D 77 (2008) 115027 [arXiv:0803.3725] [INSPIRE].

[87] P. Fayet, Supergauge invariant extension of the Higgs mechanism and a model for the electron and its neutrino, Nucl. Phys. B 90 (1975) 104 [INSPIRE].

[88] P. Fayet, Supersymmetry and weak, electromagnetic and strong interactions, Phys. Lett. B 64 (1976) 159 [INSPIRE].

[89] P. Fayet, Spontaneously broken supersymmetric theories of weak, electromagnetic and strong interactions, Phys. Lett. B 69 (1977) 489 [INSPIRE].

[90] P. Fayet, Relations between the masses of the superpartners of leptons and quarks, the Goldstino couplings and the neutral currents, Phys. Lett. B 84 (1979) 416 [INSPIRE].

[91] H.P. Nilles, M. Srednicki and D. Wyler, Weak interaction breakdown induced by supergravity, Phys. Lett. B 120 (1983) 346 [INSPIRE].

[92] J.M. Frere, D.R.T. Jones and S. Raby, Fermion masses and induction of the weak scale by supergravity, Nucl. Phys. B 222 (1983) 11 [INSPIRE].

[93] J.P. Derendinger and C.A. Savoy, Quantum effects and $\mathrm{SU}(2) \times \mathrm{U}(1)$ breaking in supergravity gauge theories, Nucl. Phys. B 237 (1984) 307 [INSPIRE].

[94] J.R. Ellis, J.F. Gunion, H.E. Haber, L. Roszkowski and F. Zwirner, Higgs bosons in a nonminimal supersymmetric model, Phys. Rev. D 39 (1989) 844 [InSPIRE].

[95] M. Drees, Supersymmetric models with extended Higgs sector, Int. J. Mod. Phys. A 4 (1989) 3635 [INSPIRE].

[96] U. Ellwanger, C. Hugonie and A.M. Teixeira, The next-to-minimal supersymmetric standard model, Phys. Rept. 496 (2010) 1 [arXiv:0910.1785] [INSPIRE].

[97] M. Maniatis, The next-to-minimal supersymmetric extension of the standard model reviewed, Int. J. Mod. Phys. A 25 (2010) 3505 [arXiv:0906.0777] [InSPIRE].

[98] J.E. Kim and H.P. Nilles, The $\mu$ problem and the strong CP problem, Phys. Lett. B 138 (1984) 150 [inSPIRE].

[99] Delphi, OPAl, Aleph, LeP Working Group for Higgs Boson Searches and L3 collaborations, S. Schael et al., Search for neutral MSSM Higgs bosons at LEP, Eur. Phys. J. C 47 (2006) 547 [hep-ex/0602042] [INSPIRE]. 
[100] Z. Kang, J. Li and T. Li, On naturalness of the MSSM and NMSSM, JHEP 11 (2012) 024 [arXiv:1201.5305] [INSPIRE].

[101] J.-J. Cao, Z.-X. Heng, J.M. Yang, Y.-M. Zhang and J.-Y. Zhu, A SM-like Higgs near $125 \mathrm{GeV}$ in low energy SUSY: a comparative study for MSSM and NMSSM, JHEP 03 (2012) 086 [arXiv: 1202.5821] [inSPIRE].

[102] J. Cao, Z. Heng, J.M. Yang and J. Zhu, Status of low energy SUSY models confronted with the LHC $125 \mathrm{GeV}$ Higgs data, JHEP 10 (2012) 079 [arXiv:1207.3698] [INSPIRE].

[103] T. Kobayashi, H. Makino, K.-I. Okumura, T. Shimomura and T. Takahashi, TeV scale mirage mediation in NMSSM, JHEP 01 (2013) 081 [arXiv:1204.3561] [INSPIRE].

[104] K.S. Jeong, Y. Shoji and M. Yamaguchi, Singlet-doublet Higgs mixing and its implications on the Higgs mass in the PQ-NMSSM, JHEP 09 (2012) 007 [arXiv:1205.2486] [INSPIRE].

[105] K.S. Jeong, Y. Shoji and M. Yamaguchi, Higgs mixing in the NMSSM and light Higgsinos, JHEP 11 (2014) 148 [arXiv:1407.0955] [INSPIRE].

[106] K. Agashe, Y. Cui and R. Franceschini, Natural islands for a $125 \mathrm{GeV}$ Higgs in the scale-invariant NMSSM, JHEP 02 (2013) 031 [arXiv:1209.2115] [INSPIRE].

[107] K. Choi, S.H. Im, K.S. Jeong and M. Yamaguchi, Higgs mixing and diphoton rate enhancement in NMSSM models, JHEP 02 (2013) 090 [arXiv:1211.0875] [INSPIRE].

[108] K. Kowalska, S. Munir, L. Roszkowski, E.M. Sessolo, S. Trojanowski and Y.-L.S. Tsai, Constrained next-to-minimal supersymmetric standard model with a $126 \mathrm{GeV}$ Higgs boson: a global analysis, Phys. Rev. D 87 (2013) 115010 [arXiv:1211.1693] [INSPIRE].

[109] T. Gherghetta, B. von Harling, A.D. Medina and M.A. Schmidt, The scale-invariant NMSSM and the $126 \mathrm{GeV}$ Higgs boson, JHEP 02 (2013) 032 [arXiv:1212.5243] [INSPIRE].

[110] T. Gherghetta, B. von Harling, A.D. Medina and M.A. Schmidt, The price of being SM-like in SUSY, JHEP 04 (2014) 180 [arXiv:1401.8291] [INSPIRE].

[111] R. Barbieri, D. Buttazzo, K. Kannike, F. Sala and A. Tesi, Exploring the Higgs sector of a most natural NMSSM, Phys. Rev. D 87 (2013) 115018 [arXiv:1304.3670] [INSPIRE].

[112] M. Badziak, M. Olechowski and S. Pokorski, New regions in the NMSSM with a $125 \mathrm{GeV}$ Higgs, JHEP 06 (2013) 043 [arXiv: 1304.5437] [InSPIRE].

[113] M. Farina, M. Perelstein and B. Shakya, Higgs couplings and naturalness in $\lambda$-SUSY, JHEP 04 (2014) 108 [arXiv: 1310.0459] [INSPIRE].

[114] M. Papucci, J.T. Ruderman and A. Weiler, Natural SUSY endures, JHEP 09 (2012) 035 [arXiv:1110.6926] [INSPIRE].

[115] H. Baer, V. Barger, P. Huang and X. Tata, Natural supersymmetry: LHC, dark matter and ILC searches, JHEP 05 (2012) 109 [arXiv:1203.5539] [INSPIRE].

[116] H. Baer, V. Barger, P. Huang, A. Mustafayev and X. Tata, Radiative natural SUSY with a 125 GeV Higgs boson, Phys. Rev. Lett. 109 (2012) 161802 [arXiv: 1207.3343] [INSPIRE].

[117] H. Baer, V. Barger, P. Huang, D. Mickelson, A. Mustafayev and X. Tata, Radiative natural supersymmetry: reconciling electroweak fine-tuning and the Higgs boson mass, Phys. Rev. D 87 (2013) 115028 [arXiv:1212.2655] [INSPIRE].

[118] H. Baer et al., Radiatively-driven natural supersymmetry at the LHC, JHEP 12 (2013) 013 [Erratum ibid. 06 (2015) 053] [arXiv: 1310.4858] [INSPIRE]. 
[119] O. Buchmueller and J. Marrouche, Universal mass limits on gluino and third-generation squarks in the context of natural-like SUSY spectra, Int. J. Mod. Phys. A 29 (2014) 1450032 [arXiv: 1304.2185] [INSPIRE].

[120] G.D. Kribs, A. Martin and A. Menon, Natural supersymmetry and implications for Higgs physics, Phys. Rev. D 88 (2013) 035025 [arXiv:1305.1313] [InSPIRE].

[121] J.A. Evans, Y. Kats, D. Shih and M.J. Strassler, Toward full LHC coverage of natural supersymmetry, JHEP 07 (2014) 101 [arXiv: 1310.5758] [INSPIRE].

[122] S.P. Martin, Nonuniversal gaugino masses and seminatural supersymmetry in view of the Higgs boson discovery, Phys. Rev. D 89 (2014) 035011 [arXiv:1312.0582] [INSPIRE].

[123] T. Cohen, J. Kearney and M. Luty, Natural supersymmetry without light Higgsinos, Phys. Rev. D 91 (2015) 075004 [arXiv:1501.01962] [INSPIRE].

[124] M. Asano and T. Higaki, Natural supersymmetric spectrum in mirage mediation, Phys. Rev. D 86 (2012) 035020 [arXiv: 1204.0508] [INSPIRE].

[125] G. Bélanger, U. Ellwanger, J.F. Gunion, Y. Jiang, S. Kraml and J.H. Schwarz, Higgs bosons at 98 and $125 \mathrm{GeV}$ at LEP and the LHC, JHEP 01 (2013) 069 [arXiv:1210.1976] [INSPIRE].

[126] D.G. Cerdeno, P. Ghosh and C.B. Park, Probing the two light Higgs scenario in the NMSSM with a low-mass pseudoscalar, JHEP 06 (2013) 031 [arXiv: 1301.1325] [INSPIRE].

[127] B. Bhattacherjee, M. Chakraborti, A. Chakraborty, U. Chattopadhyay, D. Das and D.K. Ghosh, Implications of the $98 \mathrm{GeV}$ and $125 \mathrm{GeV}$ Higgs scenarios in nondecoupling supersymmetry with updated ATLAS, CMS and PLANCK data, Phys. Rev. D 88 (2013) 035011 [arXiv: 1305.4020] [INSPIRE].

[128] R. Barbieri, D. Buttazzo, K. Kannike, F. Sala and A. Tesi, One or more Higgs bosons?, Phys. Rev. D 88 (2013) 055011 [arXiv: 1307.4937] [INSPIRE].

[129] M. Drees, A supersymmetric explanation of the excess of Higgs-like events at LEP, Phys. Rev. D 71 (2005) 115006 [hep-ph/0502075] [INSPIRE].

[130] M. Drees, A supersymmetric explanation of the excess of Higgs-like events at the LHC and at LEP, Phys. Rev. D 86 (2012) 115018 [arXiv:1210.6507] [InSPIRE].

[131] R. Dermisek and J.F. Gunion, Consistency of LEP event excesses with an $h \rightarrow$ aa decay scenario and low-fine-tuning NMSSM models, Phys. Rev. D 73 (2006) 111701 [hep-ph/0510322] [INSPIRE].

[132] R. Dermisek and J.F. Gunion, The NMSSM solution to the fine-tuning problem, precision electroweak constraints and the largest LEP Higgs event excess, Phys. Rev. D 76 (2007) 095006 [arXiv: 0705.4387] [INSPIRE].

[133] F. Staub et al., Higgs mass predictions of public NMSSM spectrum generators, arXiv: 1507.05093 [INSPIRE].

[134] H. Abe, T. Higaki and T. Kobayashi, KKLT type models with moduli-mixing superpotential, Phys. Rev. D 73 (2006) 046005 [hep-th/0511160] [InSPIRE].

[135] K. Choi, K.S. Jeong and K.-I. Okumura, Flavor and CP conserving moduli mediated SUSY breaking in flux compactification, JHEP 07 (2008) 047 [arXiv: 0804.4283] [INSPIRE].

[136] Y. Kawamura, T. Kobayashi and J. Kubo, Soft scalar mass sum rule in gauge Yukawa unified models and its superstring interpretation, Phys. Lett. B 405 (1997) 64 [hep-ph/9703320] [INSPIRE]. 
[137] T. Kobayashi, J. Kubo, M. Mondragon and G. Zoupanos, Constraints on finite soft supersymmetry breaking terms, Nucl. Phys. B 511 (1998) 45 [hep-ph/9707425] [INSPIRE].

[138] O. Lebedev, H.P. Nilles and M. Ratz, A note on fine-tuning in mirage mediation, hep-ph/0511320 [INSPIRE].

[139] A. Riotto and E. Roulet, Vacuum decay along supersymmetric flat directions, Phys. Lett. B 377 (1996) 60 [hep-ph/9512401] [INSPIRE].

[140] A. Kusenko, P. Langacker and G. Segre, Phase transitions and vacuum tunneling into charge and color breaking minima in the MSSM, Phys. Rev. D 54 (1996) 5824 [hep-ph/9602414] [INSPIRE].

[141] A. Kusenko and P. Langacker, Is the vacuum stable?, Phys. Lett. B 391 (1997) 29 [hep-ph/9608340] [INSPIRE].

[142] K. Nakayama, N. Yokozaki and K. Yonekura, Relaxing the Higgs mass bound in singlet extensions of the MSSM, JHEP 11 (2011) 021 [arXiv:1108.4338] [INSPIRE].

[143] U. Ellwanger and C. Hugonie, NMHDECAY 2.0: an updated program for sparticle masses, Higgs masses, couplings and decay widths in the NMSSM, Comput. Phys. Commun. 175 (2006) 290 [hep-ph/0508022] [INSPIRE].

[144] G. Bélanger, F. Boudjema, C. Hugonie, A. Pukhov and A. Semenov, Relic density of dark matter in the NMSSM, JCAP 09 (2005) 001 [hep-ph/0505142] [INSPIRE].

[145] U. Ellwanger, J.F. Gunion and C. Hugonie, NMHDECAY: a fortran code for the Higgs masses, couplings and decay widths in the NMSSM, JHEP 02 (2005) 066 [hep-ph/0406215] [INSPIRE].

[146] Y. Kanehata, T. Kobayashi, Y. Konishi, O. Seto and T. Shimomura, Constraints from unrealistic vacua in the next-to-minimal supersymmetric standard model, Prog. Theor. Phys. 126 (2011) 1051 [arXiv:1103.5109] [INSPIRE].

[147] T. Kobayashi, T. Shimomura and T. Takahashi, Constraining the Higgs sector from false vacua in the next-to-minimal supersymmetric standard model, Phys. Rev. D 86 (2012) 015029 [arXiv: 1203.4328] [INSPIRE].

[148] J.R. Ellis, K. Enqvist, D.V. Nanopoulos and F. Zwirner, Observables in low-energy superstring models, Mod. Phys. Lett. A 1 (1986) 57 [INSPIRE].

[149] R. Barbieri and G.F. Giudice, Upper bounds on supersymmetric particle masses, Nucl. Phys. B 306 (1988) 63 [INSPIRE].

[150] S. Dimopoulos and G.F. Giudice, Naturalness constraints in supersymmetric theories with nonuniversal soft terms, Phys. Lett. B 357 (1995) 573 [hep-ph/9507282] [INSPIRE].

[151] P.H. Chankowski, J.R. Ellis and S. Pokorski, The fine tuning price of LEP, Phys. Lett. B 423 (1998) 327 [hep-ph/9712234] [INSPIRE].

[152] P.H. Chankowski, J.R. Ellis, M. Olechowski and S. Pokorski, Haggling over the fine tuning price of LEP, Nucl. Phys. B 544 (1999) 39 [hep-ph/9808275] [INSPIRE].

[153] R. Barbieri and A. Strumia, About the fine tuning price of LEP, Phys. Lett. B 433 (1998) 63 [hep-ph/9801353] [INSPIRE].

[154] G.L. Kane and S.F. King, Naturalness implications of LEP results, Phys. Lett. B 451 (1999) 113 [hep-ph/9810374] [INSPIRE]. 
[155] L. Giusti, A. Romanino and A. Strumia, Natural ranges of supersymmetric signals, Nucl. Phys. B 550 (1999) 3 [hep-ph/9811386] [InSPIRE].

[156] J.A. Casas, J.R. Espinosa and I. Hidalgo, The MSSM fine tuning problem: a way out, JHEP 01 (2004) 008 [hep-ph/0310137] [INSPIRE].

[157] S. Cassel, D.M. Ghilencea and G.G. Ross, Fine tuning as an indication of physics beyond the MSSM, Nucl. Phys. B 825 (2010) 203 [arXiv:0903.1115] [InSPIRE].

[158] S. Cassel and D.M. Ghilencea, A review of naturalness and dark matter prediction for the Higgs mass in MSSM and beyond, Mod. Phys. Lett. A 27 (2012) 1230003 [arXiv:1103.4793] [INSPIRE].

[159] S. Cassel, D.M. Ghilencea and G.G. Ross, Testing SUSY at the LHC: electroweak and dark matter fine tuning at two-loop order, Nucl. Phys. B 835 (2010) 110 [arXiv:1001.3884] [INSPIRE].

[160] S. Cassel, Naturalness of electroweak physics within minimal supergravity, arXiv: 1107.4770 [INSPIRE].

[161] S.F. King and P.L. White, Resolving the constrained minimal and next-to-minimal supersymmetric standard models, Phys. Rev. D 52 (1995) 4183 [hep-ph/9505326] [INSPIRE].

[162] M. Bastero-Gil, C. Hugonie, S.F. King, D.P. Roy and S. Vempati, Does LEP prefer the NMSSM?, Phys. Lett. B 489 (2000) 359 [hep-ph/0006198] [INSPIRE].

[163] R. Dermisek and J.F. Gunion, Escaping the large fine tuning and little hierarchy problems in the next to minimal supersymmetric model and $h \rightarrow$ aa decays, Phys. Rev. Lett. 95 (2005) 041801 [hep-ph/0502105] [INSPIRE].

[164] S. Dawson et al., Working group report: Higgs boson, arXiv:1310.8361 [INSPIRE].

[165] D.M. Asner et al., ILC Higgs white paper, arXiv:1310.0763 [INSPIRE].

[166] M.E. Peskin, Estimation of LHC and ILC capabilities for precision Higgs boson coupling measurements, arXiv:1312.4974 [INSPIRE].

[167] P. Bechtle, S. Heinemeyer, O. Stål, T. Stefaniak and G. Weiglein, Probing the standard model with Higgs signal rates from the Tevatron, the LHC and a future ILC, JHEP 11 (2014) 039 [arXiv: 1403.1582] [INSPIRE].

[168] K. Fujii et al., Physics case for the International Linear Collider, arXiv:1506.05992 [INSPIRE].

[169] TLeP Design Study Working Group collaboration, M. Bicer et al., First look at the physics case of TLEP, JHEP 01 (2014) 164 [arXiv: 1308.6176] [INSPIRE].

[170] D. Carmi, A. Falkowski, E. Kuflik, T. Volansky and J. Zupan, Higgs after the discovery: a status report, JHEP 10 (2012) 196 [arXiv:1207.1718] [INSPIRE].

[171] S.F. King, M. Mühlleitner, R. Nevzorov and K. Walz, Natural NMSSM Higgs bosons, Nucl. Phys. B 870 (2013) 323 [arXiv: 1211. 5074] [InSPIRE].

[172] J.R. Ellis, M.K. Gaillard and D.V. Nanopoulos, A phenomenological profile of the Higgs boson, Nucl. Phys. B 106 (1976) 292 [InSPIRE].

[173] M.A. Shifman, A.I. Vainshtein, M.B. Voloshin and V.I. Zakharov, Low-energy theorems for Higgs boson couplings to photons, Sov. J. Nucl. Phys. 30 (1979) 711 [Yad. Fiz. 30 (1979) 1368] [INSPIRE]. 
[174] M.A. Shifman, A.I. Vainshtein and V.I. Zakharov, Remarks on Higgs boson interactions with nucleons, Phys. Lett. B 78 (1978) 443 [InSPIRE].

[175] A.I. Vainshtein, V.I. Zakharov and M.A. Shifman, Higgs particles, Sov. Phys. Usp. 23 (1980) 429 [Usp. Fiz. Nauk 131 (1980) 537] [INSPIRE].

[176] M.B. Voloshin, Once again about the role of gluonic mechanism in interaction of light Higgs boson with hadrons, Sov. J. Nucl. Phys. 44 (1986) 478 [Yad. Fiz. 44 (1986) 738] [InSPIRE].

[177] M. Farina, M. Perelstein and N. Rey-Le Lorier, Higgs couplings and naturalness, Phys. Rev. D 90 (2014) 015014 [arXiv:1305.6068] [INSPIRE].

[178] U. Ellwanger, A Higgs boson near $125 \mathrm{GeV}$ with enhanced di-photon signal in the NMSSM, JHEP 03 (2012) 044 [arXiv:1112.3548] [INSPIRE].

[179] R. Benbrik, M. Gomez Bock, S. Heinemeyer, O. Stål, G. Weiglein and L. Zeune, Confronting the MSSM and the NMSSM with the discovery of a signal in the two photon channel at the LHC, Eur. Phys. J. C 72 (2012) 2171 [arXiv:1207.1096] [INSPIRE].

[180] A. Djouadi, The anatomy of electro-weak symmetry breaking. II. The Higgs bosons in the minimal supersymmetric model, Phys. Rept. 459 (2008) 1 [hep-ph/0503173] [INSPIRE].

[181] P. Kalyniak, R. Bates and J.N. Ng, Two photon decays of scalar and pseudoscalar bosons in supersymmetry, Phys. Rev. D 33 (1986) 755 [INSPIRE].

[182] R. Bates, J.N. Ng and P. Kalyniak, Two photon decay widths of Higgs bosons in minimal broken supersymmetry, Phys. Rev. D 34 (1986) 172 [InSPIRE].

[183] LHC Higgs Cross section Working Group collaboration, A. David et al., LHC HXSWG interim recommendations to explore the coupling structure of a Higgs-like particle, arXiv: 1209.0040 [INSPIRE].

[184] LHC Higgs Cross section Working Group collaboration, J.R. Andersen et al., Handbook of LHC Higgs cross sections: 3. Higgs properties, arXiv:1307.1347 [INSPIRE].

[185] SLD Electroweak Group, Delphi, Aleph, SLD, SLD Heavy Flavour Group, OPAL, LEP Electroweak Working Group and L3 collaborations, S. Schael et al., Precision electroweak measurements on the $Z$ resonance, Phys. Rept. 427 (2006) 257 [hep-ex/0509008] [INSPIRE].

[186] G. Jungman, M. Kamionkowski and K. Griest, Supersymmetric dark matter, Phys. Rept. 267 (1996) 195 [hep-ph/9506380] [rNSPIRE].

[187] C. Marcos, M. Peiro and S. Robles, On the importance of direct detection combined limits for spin independent and spin dependent dark matter interactions, arXiv:1507.08625 [INSPIRE].

[188] S. Nakamura and M. Yamaguchi, Gravitino production from heavy moduli decay and cosmological moduli problem revived, Phys. Lett. B 638 (2006) 389 [hep-ph/0602081] [INSPIRE].

[189] M. Endo, K. Hamaguchi and F. Takahashi, Moduli-induced gravitino problem, Phys. Rev. Lett. 96 (2006) 211301 [hep-ph/0602061] [INSPIRE].

[190] M. Dine, L. Randall and S.D. Thomas, Baryogenesis from flat directions of the supersymmetric standard model, Nucl. Phys. B 458 (1996) 291 [hep-ph/9507453] [InSPIRE].

[191] A.D. Linde, Relaxing the cosmological moduli problem, Phys. Rev. D 53 (1996) 4129 [hep-th/9601083] [INSPIRE]. 
[192] K. Nakayama, F. Takahashi and T.T. Yanagida, On the adiabatic solution to the Polonyi/moduli problem, Phys. Rev. D 84 (2011) 123523 [arXiv:1109. 2073] [INSPIRE].

[193] M. Dine, R. Kitano, A. Morisse and Y. Shirman, Moduli decays and gravitinos, Phys. Rev. D 73 (2006) 123518 [hep-ph/0604140] [INSPIRE].

[194] M. Bose, M. Dine and P. Draper, Moduli or not, Phys. Rev. D 88 (2013) 023533 [arXiv: 1305.1066] [INSPIRE].

[195] D.H. Lyth and E.D. Stewart, Cosmology with a TeV mass GUT Higgs, Phys. Rev. Lett. 75 (1995) 201 [hep-ph/9502417] [INSPIRE].

[196] D.H. Lyth and E.D. Stewart, Thermal inflation and the moduli problem, Phys. Rev. D 53 (1996) 1784 [hep-ph/9510204] [INSPIRE].

[197] K. Choi, W.-I. Park and C.S. Shin, Cosmological moduli problem in large volume scenario and thermal inflation, JCAP 03 (2013) 011 [arXiv:1211.3755] [INSPIRE].

[198] M. Kawasaki and K. Nakayama, Baryon asymmetry in heavy moduli scenario, Phys. Rev. D 76 (2007) 043502 [arXiv: 0705.0079] [INSPIRE].

[199] H. Hattori, T. Kobayashi, N. Omoto and O. Seto, Entropy production by domain wall decay in the NMSSM, Phys. Rev. D 92 (2015) 103518 [arXiv:1510.03595] [INSPIRE].

[200] N. Arkani-Hamed, A. Delgado and G.F. Giudice, The well-tempered neutralino, Nucl. Phys. B 741 (2006) 108 [hep-ph/0601041] [InSPIRE].

[201] D.G. Cerdeno, C. Hugonie, D.E. Lopez-Fogliani, C. Muñoz and A.M. Teixeira, Theoretical predictions for the direct detection of neutralino dark matter in the NMSSM, JHEP 12 (2004) 048 [hep-ph/0408102] [INSPIRE].

[202] LUX collaboration, D.S. Akerib et al., First results from the LUX dark matter experiment at the Sanford Underground Research Facility, Phys. Rev. Lett. 112 (2014) 091303 [arXiv: 1310.8214$]$ [INSPIRE].

[203] COUPP collaboration, E. Behnke et al., First dark matter search results from a $4 \mathrm{~kg} \mathrm{CF}_{3} I$ bubble chamber operated in a deep underground site, Phys. Rev. D 86 (2012) 052001

[Erratum ibid. D 90 (2014) 079902] [arXiv:1204.3094] [INSPIRE].

[204] XENON100 collaboration, E. Aprile et al., Limits on spin-dependent WIMP-nucleon cross sections from 225 live days of XENON100 data, Phys. Rev. Lett. 111 (2013) 021301 [arXiv:1301.6620] [INSPIRE].

[205] Fermi-LAT collaboration, M. Ackermann et al., Dark matter constraints from observations of 25 Milky Way satellite galaxies with the Fermi Large Area Telescope, Phys. Rev. D 89 (2014) 042001 [arXiv:1310.0828] [INSPIRE]. 